\title{
On the radiation tolerance of CMOS Monolithic Active Pixel Sensors with partially depleted active volume
}

\author{
Schriftliche Habilitationsleistung \\ Michael Deveaux \\ Goethe Universität Frankfurt am Main, Institut für Kernphysik \\ Max-von-Laue-Straße, D-60438 Frankfurt am Main, Germany
}

30. Januar 2019

Vorgelegt beim Fachbereich Physik der Goethe-Universität Frankfurt am Main 



\begin{abstract}
CMOS Monolithic Active Pixel Sensors for charged particle tracking (CPS) form are ultralight and highly granular silicon pixel detectors suited for highly sensitive charged particle tracking. Unlike to most other silicon radiation detectors, they rely on standard CMOS technology. This cost efficient approach allows for building particularly small and thin pixels but also introduced, until recently, substantially constraints on the design of the sensors. The most important among them is the missing compatibility with the use of PMOS transistors and depleted charge collection diodes in the pixel. Traditional CPS were thus first of all suited for vertex detectors of relativistic heavy ion and particle physics experiments, which require highest tracking accuracy in combination with moderate time resolution and radiation tolerance.

This work reviews the $R \& D$ on understanding and improving the radiation tolerance of traditional CPS with non- and partially depleted active medium as pioneered by the MIMOSAseries developed by the IPHC Strasbourg. It introduces the specific measurement methods used to assess the radiation tolerance of those non-standard pixels. Moreover, it discusses the major mechanisms of radiation damage and procedures for radiation hardening, which allowed to extend the radiation tolerance of the devices by more than an order of magnitude.
\end{abstract}




\section{Acknowledgements}

The author wishes to thank Prof. J. Stroth (Goethe Universtity Frankfurt) and Marc Winter (PICSEL group, IPHC Strasbourg [1]) and the members of their working groups for their friendship and continuous support. Many of the studies presented were carried out together with students of the AG Prof. Stroth. Special thanks to S. Amar-Youcef, A. Büdenbender, T. Bus, D. Doering, M. Domachowski, B. Linnik, S. Ottersbach, S. Strohauer, P. Scharrer and A. Yazgili for participating in the studies and for many interesting discussions. The sensors tested were provided by the PICSEL group of IPHC Strasbourg, which also provided valuable help for setting up the test infrastructure and irradiating sensors.

This work was supported by the BMBF, HIC for FAIR and the Helmholtz Gesellschaft für Schwerionenforschung (GSI) and the HGS-HIRe. M. Deveaux was supported by the MainCampus Stipendiatenwerk der Stiftung Polytechnische Gesellschaft Frankfurt am Main. 



\section{Contents}

\begin{tabular}{lll}
\hline 1 & Introduction & 4
\end{tabular}

\begin{tabular}{|lll}
2 & Mechanisms of radiation damage in CMOS devices & 7
\end{tabular}

$2.1 \quad$ Ionizing radiation damage $\ldots \ldots \ldots \ldots \ldots \ldots \ldots \ldots$

$2.2 \quad$ Non-ionizing radiation damage $\ldots \ldots \ldots \ldots$. . . . . . . . . . . . . 10

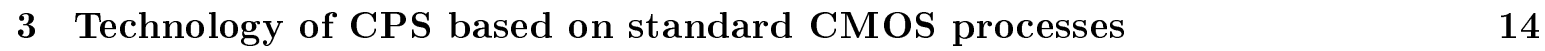

3.1 Classical CPS . . . . . . . . . . . . . . . . . . . . . . . 14

$3.1 .1 \quad$ Fundamental design considerations . . . . . . . . . . . . . . . . . . 14

3.1 .2 The classical sensing element . . . . . . . . . . . . . . . . . . 15

3.1 .3 The 3T-amplifier . . . . . . . . . . . . . . . . . . . 16

3.1.4 The rolling-shutter readout scheme . . . . . . . . . . . . . . . . . 18

3.2 CPS with accelerated time resolution and rate capability . . . . . . . . . . . 18

$3.2 .1 \quad$ Design challenges and approaches for solutions . . . . . . . . . . 18

$3.2 .2 \quad$ Eliminating the dead-time - The Self-Bias-Pixel . . . . . . . . . . . . . 19

$3.2 .3 \quad$ From sequential to column parallel - The clamping-pixel . . . . . . . . 20

$\begin{array}{lll}3.3 & \text { Performances of selected CMOS sensors based on standard CMOS processes } & 21\end{array}$

4 Methods for observing radiation damage 23

4.1 Setting up the testing hardware . . . . . . . . . . . . . . . . . . 23

4.2 Irradiating the sensors $\ldots \ldots \ldots \ldots \ldots \ldots \ldots$. . . . . . . . . . . . . . 24

$4.2 .1 \quad$ Irradiating CPS with X-rays . . . . . . . . . . . . . . . . . 24

4.2 .2 Irradiating CPS with neutrons . . . . . . . . . . . . . . . 24

4.3 Accessible quantities and their measurement . . . . . . . . . . . . . . . . . . . . . 25

$4.3 .1 \quad$ Chip temperature . . . . . . . . . . . . . . . . . 25

4.3 .2 Leakage currents . . . . . . . . . . . . . . . . . . 25

$4.3 .3 \quad$ Pixel noise (analogue readout) . . . . . . . . . . . . . 26

4.3 .3 .1 High frequency noise $\ldots \ldots \ldots \ldots$. . . . . . . . . 26

4.3 .3 .2 Common mode noise . . . . . . . . . . . . . . . 27

4.3.3.3 Random Telegraph Signal (RTS) . . . . . . . . . . . . . . . . . . . 27

$4.3 .3 .4 \quad$ Fixed pattern noise $\ldots \ldots \ldots \ldots . \ldots \ldots 27$

$\begin{array}{lll}4.3 .4 & \text { The } & 55 \\ \end{array}$

$4.3 .5 \quad$ The ${ }^{90} \mathrm{Sr}$ amplitude spectrum - signal charge and SNR . . . . . . . . 29

4.3 .6 Pixel clearing constant . . . . . . . . . . . . . . . . 30

\begin{tabular}{|lll}
5 & Effects of integrated ionizing radiation & 31
\end{tabular}

5.1 Increase of leakage currents and shot noise $\ldots \ldots \ldots \ldots$. . . . . . . 31

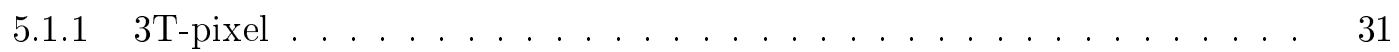

$5.1 .1 .1 \quad$ Leakage current $\ldots \ldots \ldots \ldots$

5.1 .1 .2 Noise $\ldots \ldots \ldots \ldots \ldots \ldots \ldots \ldots$

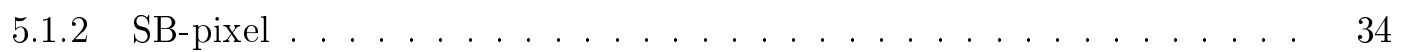

$5.1 .2 .1 \quad$ Leakage current $\ldots \ldots \ldots \ldots$. . . . . . . . . . . . 34 
5.1 .2 .2 Signal clearing . . . . . . . . . . . . . . . . 35

5.1 .2 .3 Noise $\ldots \ldots \ldots \ldots \ldots$. . . . . . . . . . . . . . . . 37

$5.1 .3 \quad$ Clamping pixel $\ldots \ldots \ldots \ldots$

5.2 Effect of ionizing radiation to the charge collection $\ldots \ldots \ldots \ldots$. . . . . 39

5.3 Results from beam test campaigns . . . . . . . . . . . . . . . . . . 40

6 Effects of integrated non-ionizing radiation $\quad 41$

6.1 Sensors with $10 \Omega \cdot \mathrm{cm}$ epitaxial layer . . . . . . . . . . . . . . . . . 41

$6.1 .1 \quad$ Charge collection efficiency, gain and noise . . . . . . . . . . . . . 41

6.1 .2 Leakage currents . . . . . . . . . . . . . . . . . . . 44

6.1.3 Radiation hardening strategies for CPS with low resistivity active medium 45

6.1.3.1 Thickness of the active medium. . . . . . . . . . 45

6.1 .3 .2 Pixels with multiple, parallel collection diodes . . . . . . . 45

$6.1 .3 .3 \quad$ Pixel pitch . . . . . . . . . . . . . . . . . . . 46

6.1 .3 .4 Doping gradients . . . . . . . . . . . . . . . . 47

6.2 Sensors with high resistivity $\sim 1 \mathrm{k} \Omega \cdot \mathrm{cm}$ epitaxial layer $\ldots \ldots \ldots \ldots$

$6.2 .1 \quad$ Charge Collection Efficiency . . . . . . . . . . . . . . . . 49

6.2 .2 Noise and leakage current . . . . . . . . . . . . . . . . . . . . 53

6.3 Results from beam tests . . . . . . . . . . . . . . . . . . . . . . . . . . 54

6.4 Outlook: Depleted MAPS $\ldots \ldots \ldots \ldots \ldots \ldots$

$\begin{array}{lll}6.4 .1 & \text { Depleted CPS, fundamental considerations and technological approach } 56\end{array}$

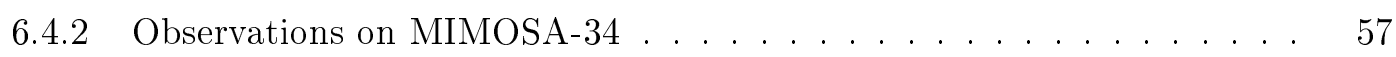

6.4 .3 Observations of PIPPER-2 $\ldots \ldots \ldots \ldots \ldots$. . . . . . . . . . 59

\begin{tabular}{|lll}
\hline 7 & Thermal Annealing & 63
\end{tabular}

8 Specific radiation damage effects $\quad 65$

8.1 Random Telegraph Signal . . . . . . . . . . . . . . . . . . . . . . . . . . . . . . . . . 65

8.1 .1 Introduction . . . . . . . . . . . . . . . . . . 65

8.1 .2 RTS in the leakage current of charge collection diodes . . . . . . . . 66

8.1.2.1 $\quad$ Parametrization, properties and detection of RTS . . . . . 66

8.1 .2 .2 Number of RTS-pixel as a function of radiation doses . . . . 68

$8.1 .2 .3 \quad$ Impact of the diode RTS on 3T-pixels $\ldots \ldots \ldots 71$

8.1 .2 .4 Impact of diode RTS on SB-pixels . . . . . . . . . . . . . . . . . . . 71

8.1 .3 RTS in MOSFET-transistors . . . . . . . . . . . . . . 73

$8.1 .3 .1 \quad$ Properties of the RTS $\ldots \ldots \ldots \ldots \ldots$

$8.1 .3 .2 \quad$ Impact on CPS $\ldots \ldots \ldots \ldots \ldots \ldots$

8.1 .3 .3 Impact of radiation damage on RTS in MOSFETs . . . . . . 75

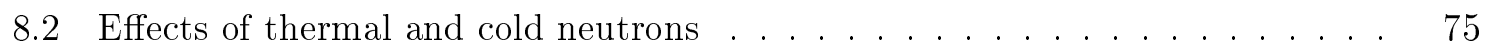

8.3 Radiation damage caused by relativistic heavy ions . . . . . . . . . . . 78

$8.3 .1 \quad$ Single event upsets and latch-up . . . . . . . . . . . . . . . . . 79

$8.3 .2 \quad$ Integrated displacement damage caused by heavy ions . . . . . . . . 81

\begin{tabular}{lll}
\hline 9 & Summary and conclusion & 83
\end{tabular} 


\section{Introduction}

CMOS Monolithic Active Pixel Sensors (CPS or MAPS) are silicon pixel sensors, which can be produced with standard CMOS processes. By definition, active pixels are formed from a sensing element and a pre-amplifier. The pixels of modern CPS may moreover integrate a full shaper and discriminator chain.

CPS were initially developed for a use as cost efficient optical imagers and became increasingly successful in the field of digital photography. Their main economical advantage consists in the fact that they may be realized as a cheap monolithic CMOS chip while more traditional imagers like CCDs required their sensing and readout units to be placed on two separate chips. The latter was done to solve a technological conflict: Photo diodes are preferably fully depleted, which calls for lowly doped silicon and high voltages. Building the highly integrated circuits needed for amplifying and processing the diode's signal calls for the use of CMOS technology, which bases typically on comparably highly doped silicon and is compatible with low voltages only. Building a system from two separate chips allows to use the most suited technology for both, sensors and amplifiers. However, it comes also with the costs and complexity of building and interconnecting both subsystems.

CPS integrate both building blocks to a single CMOS chip, which reduces cost and complexity. However, the sensing elements are designed with CMOS processes, which were initially neither intended nor optimized for this purpose. Among the compromises introduced by this design choice were the need to use non-depleted, or only partially depleted, sensing elements, which are less performing than their optimized, fully depleted counter parts. Moreover, the use of PMOS transistors in the pixels was initially not possible, which constrained the design of the on-pixel amplifier circuits. Despite those compromises, very satisfactory performances were reached.

Already short after the invention of CPS, it was recognized that those sensors are sensitive to minimum ionizing particles and thus suited for a use in tracking detectors for relativistic heavy ion and particle physics [2]. Despite its above mentioned limitations, the technology was considered to provide an interesting potential. The size of pixels of CPS may amount few $\mu \mathrm{m}$ only, which is realized in state of the art digital cameras. In charged particle detection, small pixels measure the impact point of minimum ionizing particles with outstanding precision. Moreover, CPS are by themselves very thin, energy efficient and do not require supporting readout chips. This allows to integrate them into very light sensor systems, which minimizes the multiple coulomb scattering of minimum ionizing particles in the sensor material and thus the feedback of the instrument on the particle trajectories to be measured.

Indeed, first CPS prototypes showed a single point resolution of $1-2 \mu \mathrm{m}$ and a detection efficiency close to $100 \%[3]$ in a $\sim 100 \mathrm{GeV} / \mathrm{c}$ pion beam at the CERN-SPS. Those excellent results were soon be reproduced with a full size CPS up to $10^{6}$ pixels on active areas as large as $4 \mathrm{~cm}^{2}$ [3, 4]. Soon after, the feasibility of thinning the sensors to a thickness of $50 \mu \mathrm{m}$ without loss of performance was demonstrated. On the negative side, the time resolution of early CPS was in the order of $10 \mathrm{~ms}$, which compares with the $25 \mathrm{~ns}$ of classical hybrid pixel sensors. The radiation tolerance of CPS was initially fully unknown and came out to be rather moderate for the first sensor generation. 
Their weakness in terms of time resolution and radiation tolerance hampered first generation CPS from reaching the rate capability of the very fast and radiation hard hybrid pixel sensors as used e.g. at the early LHC. However, given their strong points, CPS came out to complement those pixels and to form the technology of choice for experiments, which call for highest precision of the trajectory measurement but allow for compromises in terms of rate capability. This holds e.g. for the vertex detector systems of electron/positron collider experiments and of relativistic heavy ion experiments aiming for the measurement of open charm particles.

One of the driving forces pushing this idea forward was the PICSEL group [1] of the IPHC Strasbourg (previously IReS and LEPSI), which designed and tested the above mentioned first CPS prototypes. This work aimed initially for the TESLA experiment [5, 6], which became the Future International Linear Collider (ILC) [7] later on. The potential of the technology was rapidly recognized outside the ILC community and a first working vertex detector based on CPS was finally realized in the heavy ion experiment STAR, which is carried out at the RHIC collider [8]. In parallel, an R\&D program aiming for a use of CPS in the Micro Vertex Detector of the Compressed Baryonic Matter experiment (CBM) [9, 10, 11 was started. The related prototype was converted into the Small Acceptance Vertex Detector of NA61, which realized a first CPS vertex detector in a fixed target geometry [12, 13, 14].

Using CPS in the above mentioned applications required to improve their rate capability and time resolution by orders of magnitude. Moreover, it was necessary to establish their radiation tolerance and to extend it substantially. This task was complicated by the specific features of CPS. Conventional CPS use a non-depleted, low resistivity, p-doped active medium and collect their signal charge dominantly by means of thermal diffusion. Due to those properties, CPS feature a potentially unique device physics and most established knowledge on radiation tolerance as provided e.g by the CERN RD48 and RD50 collaborations could not be applied in a straight forward way. Moreover, the active medium of CPS is as thin as $\sim 10 \mu \mathrm{m}$, which comes with a particularly small signal charge of few $100 e$ only. This makes the sensors vulnerable to particularly subtle effects injecting a noise of $\sim 10 e \mathrm{ENC}$, which were previously rarely in the focus of research. The high integration of CPS does not allow to access sensing elements and amplifiers directly or separately and any information on radiation damage had to be extracted from the output of the potentially damaged, integrated amplification chain of the device. Finally, it became only recently possible to request modifications of the CMOS processes used for device production. Therefore, any modification aiming for higher radiation hardness had to be done within the strict limits of the design rules of the non optimized commercial processes.

The focus of this work consists in reviewing the test procedures suited for testing the radiation tolerance of CPS and discussing the mechanisms limiting this tolerance in CPS with non- and partially depleted sensitive medium. Moreover, it introduces the technological approaches, which allowed to extend the radiation tolerance of CPS based on classical, double well CMOS processes by more than one order of magnitude and such to reach the performances required for using them in a first set of heavy ion experiments. Note that, due to this focus, this work does not cover the numerous and promising attempts to build a next generation of CPS based on next generation CMOS processes, which allow for using PMOS transistors and 
depleting the pixels (see e.g. [16, 17, 18, 19, 20]).

The document is structured as follows: In Sec. 2, a brief introduction on established radiation damage effects will provided. Sec. 3 introduces the technological approach of of CPS relying on traditional CMOS processes. An overview over the methods for testing the radiation tolerance of those devices is given (Sec. 47). Sec. 5 and 6 discuss the effect of integrated ionizing and non-ionizing radiation doses respectively and discusses device hardening strategies. Sec. 7 covers the effects of thermal annealing of ionizing and non-ionizing radiation damage. In Sec. 8, we will discuss specific radiation damage effects, including Random Telegraph Signal, the effects of cold (thermal) neutrons and heavy ions. Finally, the major results will be summarized. 


\section{Mechanisms of radiation damage in CMOS devices}

Radiation damage in CMOS devices can be subdivided into two major groups, which are denoted as ionizing and non-ionizing. Ionizing radiation damage is caused by a displacement of electrons from the electron shell of their atoms. It may be reversible, e.g. if the lost electron is replaced by another electron obtained from the conduction band of conductive materials; or irreversible, e.g. if a chemical binding in a molecule is permanently broken. In CMOS devices, the latter occurs dominantly at the interface between silicon and the various $\mathrm{SiO}_{2}$ structures serving for isolation. Ionizing damage is thus frequently referred to as surface damage.

Non-ionizing radiation damage is by definition ${ }^{1}$ created by interactions between the radiation and the nuclear core of atoms. The harmful consequence of non-ionizing radiation consists in the displacement of atoms from their position in the crystal lattice. The related crystal defects degrade the bulk of silicon crystals, which is referred to as bulk damage.

Most particles cause both kinds of radiation damage. Therefore, irradiated devices will typically show superimposed radiation effects. However, an understanding of the individual mechanisms is required to improve the radiation tolerance of the devices.

\subsection{Ionizing radiation damage}

Ionizing radiation damage is caused by electro-magnetic interactions, which are created by all kinds of charged particles and photons with an energy above $10 \mathrm{eV}$. The energy deposit of ionizing radiation is measured in the SI-unit Gray $(1 \mathrm{~Gy}=1 \mathrm{~J} / \mathrm{kg})$. The elder unit $1 \mathrm{rad}=0.01 \mathrm{~Gy}$ is still widely used in literature and will also be used in this work.

An ionizing energy deposit in bulk silicon is typically reversible and not of worry. Lasting damage is caused by the irradiation of $\mathrm{SiO}_{2}$ structures, which are widely used as isolators in CMOS chips. The precise mechanisms of the radiation damage generated in those structures is reviewed in [15], which is summarized in the following:

The valence and conduction band of $\mathrm{SiO}_{2}$ are separated by a wide band gap, which hampers the thermal excitation of charge carriers, but remains small as compared to the typical energy deposit caused by ionizing particles. Ionizing radiation creates electron/hole pairs in the $\mathrm{SiO}_{2}$. In the presence of an electric field, some of the pairs created are separated. The separation probability, the so-called charge yield, increases with increasing electric field. Moreover, weakly ionizing particles like $\gamma$ - and X-rays show a substantially higher charge yield than strongly ionizing particles (e.g. $\alpha$-particles). The total number of free holes 22 per $\mathrm{cm}^{2}$ $\mathrm{Si}-\mathrm{SiO}_{2}$ interface, $N_{h}$, is given with:

$$
N_{h}=f\left(E_{o x}\right) \cdot g_{0} \cdot t_{o x} \cdot D
$$

Here, $f\left(E_{o x}\right)$ denotes the (hole-) charge yield, which is function of the oxide electric field, $g_{0}=8.1 \times 10^{12} / \mathrm{cm}^{3}$ per rad for $\mathrm{SiO}_{2}$ the density of initially produced holes, $t_{o x}$ the thickness of the oxide and $D$ the dose.

\footnotetext{
${ }^{1}$ Note that this definition differs from the one used in the context of life-science and radiation protection.

${ }^{2}$ The free electrons do in first order not contribute to the relevant radiation damage effects and are ignored in the following.
} 
In CPS, as in all devices with positive bias and a bulk silicon at ground (or negative) potential, the electric fields pushes the holes toward the interface between the $\mathrm{SiO}_{2}$ structures and the bulk silicon. The mobility of the holes is low and depends strongly on temperature and oxide thickness.

Nearby the $\mathrm{Si}_{-} \mathrm{SiO}_{2}$ interface, a great number of oxygen-vacancies are found. Those are created by an out-diffusion of oxygen to the silicon and by the different lattice constants of both materials. The vacancies trap the free holes. The related trapping cross-section scales with $E_{o x}{ }^{-1 / 2}$, which counteracts to some degree the field dependent increase of the charge yield. The number of traps depends strongly on the precise production process of the device. Therefore, the fraction of trapped holes may vary between few percent and close to $100 \%$ and saturation effects are to be expected at some point.

It is of use to distinguish two major groups of trapped holes. Holes trapped near the interface in the non-conductive $\mathrm{SiO}_{2}$ are referred to as oxide charge. Their charge is always positive. Their number is reduced starting from the moment of irradiation by room temperature annealing but a significant fraction of traps may remain even after several months of annealing. Holes trapped on the conductive Si-side may create defects in the band gap of the silicon and are referred to as interface traps. Depending on their energy and the local Fermi-level (doping), their charge may be positive, neutral or negative. Interface traps are not annealed at room temperature. The charge generated by oxide charge and by charged interface traps adds up. As interface traps may show negative charge, the summed charge may be below the oxide charge or even show negative polarity.

In [21], measurement results on the defect densities are shown. As suggested by equation 1. the number of interface traps $D_{i t}$ scales linearly with the dose for a given oxide configuration and electric field. For doses well above $1 \mathrm{Mrad}$, the onset of a saturation turning into a sub-linear dependence of $D_{i t} \propto D^{2 / 3}$ is observed. At higher doses and for good silicon strip detector material, a full saturation of the positive surface charge of $d Q / d A \approx 3 \times 10^{12} / \mathrm{cm}^{2}$ is reported [22]. As expected, the number of interface traps increases with the thickness $t_{o x}$ of the oxide. For thick oxides, this increase scales with $D_{i t} \propto t_{o x}^{1.6}$ [21] and thus stronger than expected from a naive scaling of equation 1. More importantly, $D_{i t}$ shrinks dramatically for oxide thicknesses of $\lesssim 12 \mathrm{~nm}$. This effect is explained the elimination or compensation of trapped charge by tunneling of electrons from the silicon into either oxide traps or electron traps associated with trapped holes [15]. Due to the limited range of the tunnel effect, this process is most efficient for thin oxide structures.

The relevance of the radiation induced charge build up is due to the related fields. Those fields may act similar to the steering fields of field effect transistors (FET) and deform the bands nearby the interface. This may generate unwanted conductive paths, e.g. in case the flat band voltage is modified such that p-silicon separating two n-doped structures becomes locally n-conductive. Moreover, the fields generated by the radiation induced charge add literally to the steering fields of FETs and modify their threshold, which is referred to as voltage shift. One observes that the voltage shift scales with $t_{o x}{ }^{3}$ for "thick" $\mathrm{SiO}_{2}$ and that it reaches values of few $\mathrm{V} / \mathrm{Mrad}$ for the $47 \mathrm{~nm}$ thick silicon. In [15], a weaker voltage shift scaling with $t_{o x}^{1.45}$ to $t_{o x}^{1.85}$ is reported. Again, very thin silicon is less vulnerable than predicted by this scaling. 


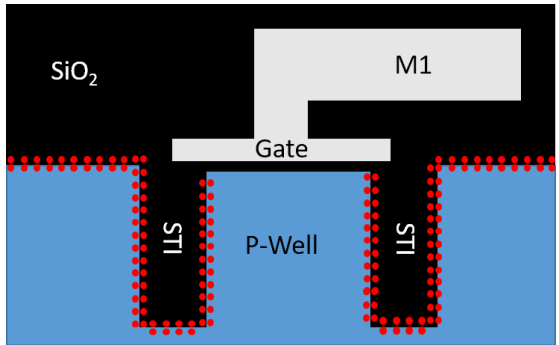

(a) Cross-section of a CMOS transistor along line A in Fig. 1c. Structures vulnerable to surface damage are indicated with red dots.

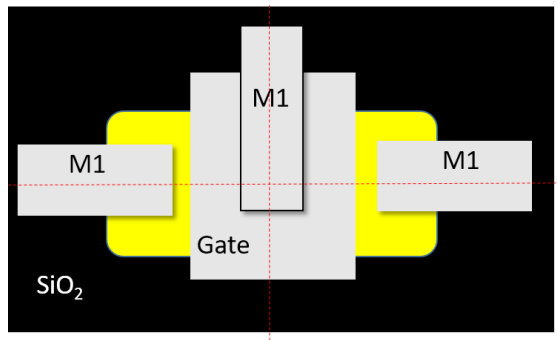

A

(c) Top view of a CMOS transistor. The lines of the cross-sections in panel $\mathrm{a}$ and $\mathrm{b}$ are indicated.

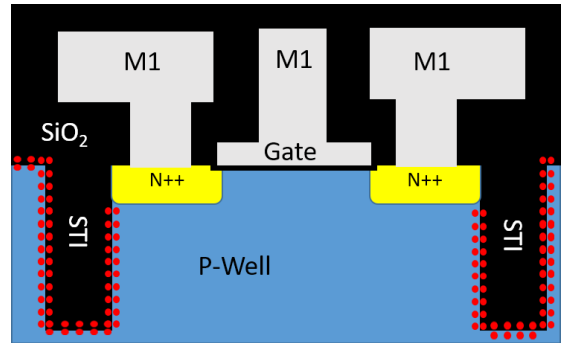

(b) Cross-section of a CMOS transistor along line B in Fig. 1c. Structures vulnerable to surface damage are indicated with red dots.

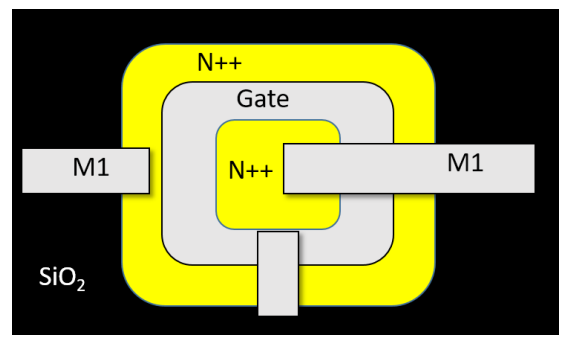

(d) Top view of an enclosed transistor. The geometry eliminates the STI aside the channel.

Figure 1: Structures vulnerable to surface damage in transistors carried out in modern CMOS processes.

In practical terms, oxide and interface charges in PMOS transistors show both a positive charge and their fields cause a negative voltage shift. In NMOS transistors, the interface charge is dominantly negative. Consequently, their fields tend to cancel out to some extend and even an inversion of the initially negative voltage shift may occur at high doses as the negatively charged interface traps start to outnumber the positively charged oxide traps. For both kinds of transistors, the voltage shift depends on the thickness of the gate oxide of the transistors. In modern deep sub-micron CMOS technologies, this thickness is typically $t_{o x} \ll 12 \mathrm{~nm}$ and thus sufficiently thin for recovering radiation damage by tunnel effect. Therefore, deep submicron CMOS processes are often considered as intrinsically radiation hard. This statement should however be taken with caution. The thickness of isolation structures others than the transistor gates is usually not reduced. Therefore, most structures remain vulnerable.

This is illustrated in Fig. 1, which shows a simplified cross-section of a FET in CMOS and indicates structures, which are typically affected by surface damage. Those structures include a thick shallow trench isolation (STI) aside the intended conductive channel of the transistor. A charge build up in those STI may create fields, which act from the side to the conductive channel of the transistor and such generate a threshold voltage shift, which is independent of the gate oxide. The magnitude of the related threshold shifts was recently studied in detail for a CMOS process with $0.18 \mu \mathrm{m}$ feature size, which is frequently used in CPS construction 


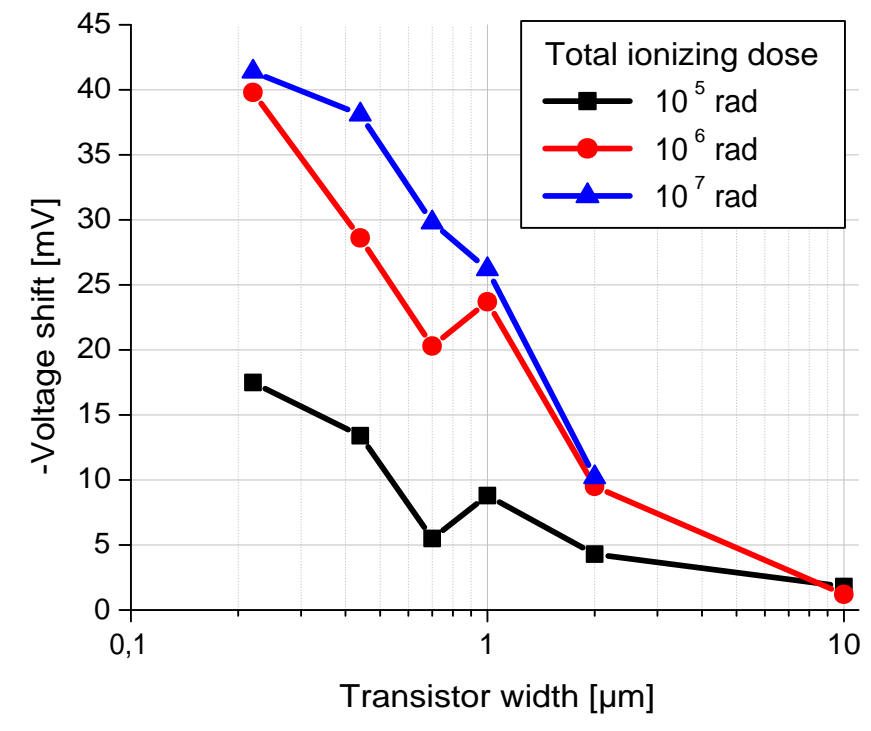

Figure 2: Threshold shifts of NMOS transistors with $0.18 \mu \mathrm{m}$ gate length as a function of the gate width and the radiation dose. The transistors were implemented in a $0.18 \mu \mathrm{m}$ CMOS process used for CPS. Data from [23], see original work for additional data.

[23. Some results of this study are displayed in Fig. 2. The figure shows the threshold voltage shift for regular NMOS transistors as a function of the width of the transistor and the radiation dose. Transistors with narrow gates show a significantly higher threshold shift than wide transistors. Moreover, the increase of the threshold shift does not scale linearly with the dose, which reflects the saturation effects for doses beyond 1 Mrad discussed above. Note that besides the voltage shifts, irradiated STI may also cause significant source to drain leakage currents, which may persist within the specified steering voltage range of the device.

To suppress threshold shifts in transistors, one may consider the use of enclosed transistors (ELT) [24, 25], which separate source and drain by a circular gate (see Fig. 1d). Thanks to this geometry, no thick and vulnerable $\mathrm{SiO}_{2}$ is located aside the gate and the threshold voltage shift is substantially reduced. This advantage has to be balanced against the higher capacitance of the gate and the substantially increased size of the transistor.

\subsection{Non-ionizing radiation damage}

Non-ionizing radiation doses are caused by an electromagnetic or strong interaction between the impinging particle and the nuclear cores of the atoms of the detector material. Once the energy transfer of an interaction exceeds $25 \mathrm{eV}$ [22], the silicon atoms are displaced out of their position in the crystal lattice. Most projectiles are substantially lighter than the atom. For reasons of momentum conservation, they transfer only a fraction of their kinetic energy. Therefore, the projectile energy needed for a displacement depends on the projectile rest mass: While silicon ions require a kinetic energy of $25 \mathrm{eV}$ to displace an individual atom and to generate a so-called point defect, the threshold is $190 \mathrm{eV}$ for protons/neutrons and $260 \mathrm{keV}$ for electrons 22 . Hard $\gamma$-rays create point defects indirectly via a generation of fast electrons, e.g. by means of Compton scattering [26]. The latter effect is however of second order in most particle physics environments.

Besides point defects, radiation may generate groups of defects. This occurs if the recoil atom is accelerated to sufficient speed to initiate secondary displacements. If so, the recoils 


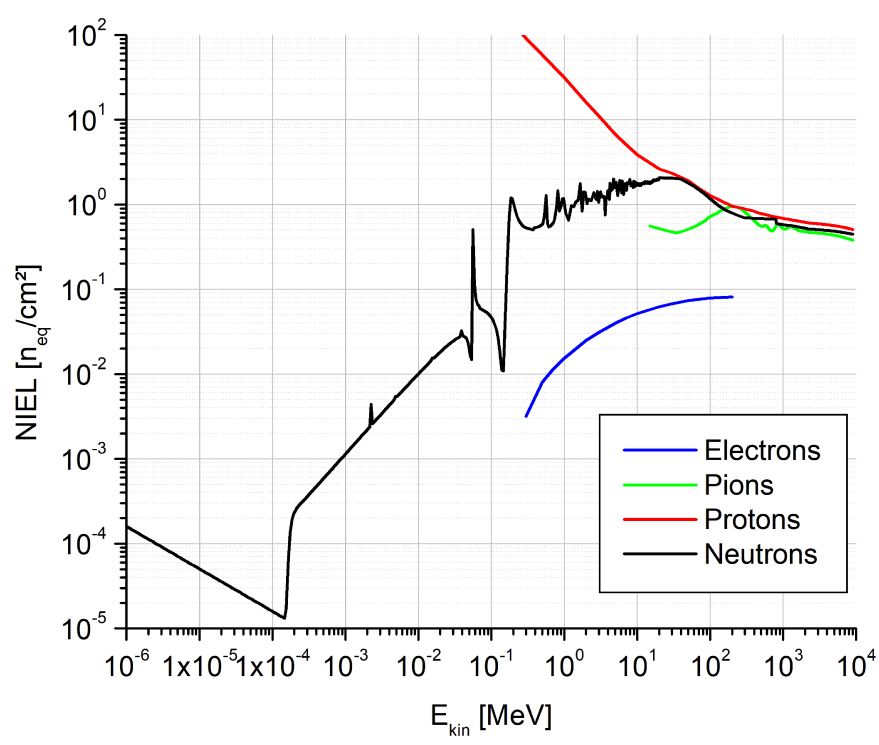

Figure 3: NIEL per particle for different particle species. Data from [28].

atom is first decelerated in a more or less smooth way by multiple coulomb scattering and hereafter stopped abruptly (Bragg peak). The latter process generates locally a large number of atomic displacements, which is referred to as defect cluster. The threshold for defect cluster generation is given with $2 \mathrm{keV}$ (Si-ion), $15 \mathrm{keV}$ (proton/neutron) and $4.6 \mathrm{MeV}$ (electron) [22].

The dosimetry on non-ionizing radiation is done by means of the so-called displacement damage cross-section $(D)$ or by the equivalent Non Ionizing Energy Loss (NIEL) [27]. By doing so, one assumes that the radiation damage caused by non-ionizing radiation scales with the non-ionizing energy deposit. This energy deposit depends on the nature and the energy of the impinging particle. It is usually normalized to the one of $1 \mathrm{MeV}$ reactor neutrons, which amounts $D=95 \mathrm{MeV}$ mb or in units of NIEL $2.03 \mathrm{keV} \mathrm{cm} \mathrm{g}^{-1}$ [29]. Based on this normalization, the NIEL caused by a radiation field is frequently expressed in the unit $\mathrm{n}_{\mathrm{eq}} / \mathrm{cm}^{2}$, which is rather convenient to compute doses from particle fluxes. The radiation damage caused by the most frequent hadrons in silicon can be normalized to units of $n_{\text {eq }} / \mathrm{cm}^{2}$ by means of tables [28]. The related data for protons, neutrons, electrons and pions are plotted in Fig. 3.

Despite of its great usefulness, the validity of the NIEL model is limited. This is because the microscopic aspects of the radiation damage are ignored. Therefore, one assumes implicitly that defect clusters show the same electric properties as multiple individual point defects. Moreover, point defects tend to bind with i) each other and/or ii) with trace elements in the silicon to secondary defects. The electric properties of those secondary effects are again mostly ignored.

Radiation induced bulk defects generate states in the band gap of the silicon. Those states ease the generation and recombination of free charge carriers in the material and thus increase the thermal leakage current of charge collection diodes. This increase is found to scale linearly with the NIEL and not to depend on the specific type of the silicon [29]. A diode with a depleted volume $V$ does thus generate a radiation induced additional leakage 
current of:

$$
\Delta I=\alpha(T) \cdot \Phi \cdot V
$$

Here, $\Phi$ represents the NIEL in units of $\mathrm{n}_{\mathrm{eq}} / \mathrm{cm}^{2}$ and the scaling factor $\alpha(T)$ depends exponentially on the temperature. It is usually given for $T=20^{\circ} \mathrm{C}$ and amounts $\alpha\left(20^{\circ} \mathrm{C}\right)=$ $4.0 \times 10^{-17} \mathrm{~A} / \mathrm{n}_{\text {eq }} / \mathrm{cm}$ after the recommended annealing of $80 \mathrm{~min}$ at $60^{\circ} \mathrm{C}$. Note that $\alpha$ is also reduced by room temperature annealing. The strict scaling of the bulk current with the NIEL suggests that this current is dominantly generated by defect clusters.

In addition, bulk damage is observed to create a loss of minority charge carriers as excited by the impinging particle. This loss may be caused by a recombination (the charge carrier is permanently lost) or by trapping (the electron is captured by a defect state but thermally released after some time). While the first case turns into a loss of signal charge, the second case may delay the arrival of the charge such that it arrives to late for contributing to the sensor signal. For non-irradiated, moderately p-doped silicon as used in CPS, the lifetime of minority charge carriers amounts $\tau_{\mathrm{eff}_{\mathrm{e}, \mathrm{h}}}=1 \mathrm{~ms}$. If the doping exceeds few $p=10^{15} / \mathrm{cm}^{3}$, $\tau_{\mathrm{eff}}{ }_{\mathrm{e}, \mathrm{h}}$ shrinks inverse to the doping concentration [30]. For irradiated sensors, the lifetime of the charge carriers until recombination or trapping scales with:

$$
\frac{1}{\tau_{\mathrm{eff}_{\mathrm{e}, \mathrm{h}}}}=\text { const } \cdot \Phi
$$

For recombination, the constant is identified with the recombination damage constant $K$. A value of $K=2.5 \times 10^{-6} \mathrm{~cm}^{2} / \mathrm{s}$ is given in [31] for electrons in P-doped silicon, which was exposed to $1 \mathrm{MeV}$ neutrons. For trapping, the constant is identified with the trapping time parameter $\beta_{e}$ and numerical values ranging from $3.4 \pm 0.3 \times 10^{-7} \mathrm{~cm}^{2} / \mathrm{s}$ to $4.7 \pm 0.4 \times 10^{-7} \mathrm{~cm}^{2} / \mathrm{s}$ (depending on the detailed P-doped material) are reported for $T=20{ }^{\circ} \mathrm{C}$ in [32].

Non-ionizing radiation modifies the effective doping of silicon, $N_{\text {eff }}=\left|N_{d}-N_{a}\right|$. Both, n- and p-dopants are absorbed and neutralized by radiation induced defects [33]. In parallel, radiation induced defects acting as effective p-dopants, are generated. N-doped silicon looses its initial doping and may become effectively p-doped silicon at some point. This effect is is referred to as charge inversion. The generation rate of the radiation induced p-dopants is reduced by about a factor of three in silicon with strong oxygen doping $\left(>10^{17} / \mathrm{cm}^{3}\right)$. Remarkably, this holds for proton and pion radiation but not for neutron radiation, which forms a prominent exception from the NIEL scaling hypothesis. The effective p-doping of irradiated silicon is observed to increase further once the silicon is annealed, which is referred to as reverse annealing. Reverse annealing is reduced by oxygen doping and may be suppressed by cooling irradiated sensors permanently to moderate negative temperatures.

For p-doped silicon, the acceptor removal is typically faster than the generation of novel states. Therefore, the effective p-doping shows a minimum at some point. Measurements for the p-doped silicon of CPS were carried out recently. According to the results, the effective doping of p-doped silicon can be described with (see e.g. [34]):

$$
N_{\text {eff }}=N_{\text {effo }}-N_{C} \cdot\left(1-\exp \left(-c \cdot \Phi_{e q}\right)\right)+g \cdot \Phi_{e q}
$$

Here, $N_{\text {effo }}$ is the initial acceptor concentration of the substrate, $N_{C}$ the concentration of the removed acceptors, $c$ the removal constant and $g$ the introduction rate of stable deep acceptors for neutron irradiation. 


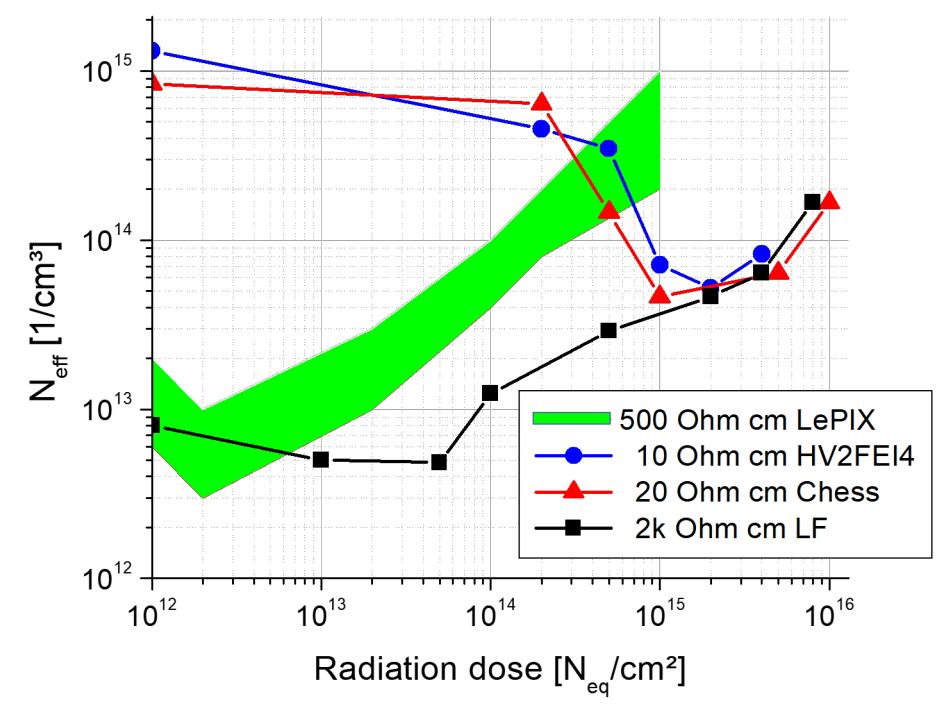

Figure 4: Effective doping of different processes used for CMOS sensors. The points at $10^{12} \mathrm{n}_{\mathrm{eq}} / \mathrm{cm}^{2}$ represents the non-irradiated devices. Data on HV2FE14, Chess and LF from [34, 35]. The LePIX - data was extracted from Fig. 4 of [36]. See original works for details on measurement strategies and uncertainties.

Measured data on the effective doping of the active volume of different CMOS processes is compiled in Fig. 4. One observes that the high initial doping of low resistivity silicon is reduced only by a small factor for doses up to $\sim 10^{14} \mathrm{n}_{\mathrm{eq}} / \mathrm{cm}^{2}$. Hereafter, a relatively massive removal sets in and a minimum of the effective doping is observed at few $\sim 10^{15} \mathrm{n}_{\text {eq }} / \mathrm{cm}^{2}$. For high resistivity silicon, the minimum of $N_{\text {eff }}$ is reached at a lower radiation dose. For higher doses, the $N_{\text {eff }}$ of the silicon presented in [34] is independent of the initial doping within error bars. For the HV2FE14, CHESS and LF data sets, a common parametrization of $c=10^{-15} / \mathrm{cm}^{2}, N_{C} / N_{\text {effo }}=0.6$ and $g_{c}=0.047 \mathrm{~cm}^{-1}$ with uncertainties of around $30 \%$ are reported. The data reported for the LePIX wafer follows qualitatively the observations of the other lines. The discrepancy may be because both measurements of the depletion depth underlying the $N_{\text {eff }}$ were done with rather different approaches (LePIX: C/V - measurements, others: TCT). At the time given, it appears that the effect of acceptor removal and acceptor generation is qualitatively present in various p-doped materials. However, the precise removal and generation rates seem dependent to some extend on the details of the material and the radiation source (protons/neutrons) [37, 38]. This is subject of ongoing research. 


\section{Technology of CPS based on standard CMOS processes}

\subsection{Classical CPS}

\subsubsection{Fundamental design considerations}

Charged particles and photons of sufficient energy generate electron/hole-pairs in silicon. Any kind of silicon radiation detector aims to separate the electrons and the holes, to collect their charge by means of a charge collection diode and to amplify this charge in order to obtain a signal. This signal has to exceed the noise of the amplification chain. In average, a radiation energy of $3.6 \mathrm{eV}$ is needed to generate an e/h pair [22].

$\mathrm{X}$-rays and fast charged particles interact with silicon in different ways. X-rays tend to concentrate their full energy in a tiny volume and thus create a localized charge cloud. Fast charged particles (minimal ionising particles, MIPs) are typically not stopped in the sensor and generate $\sim 80 \mathrm{e} / \mathrm{h}$ pairs per $\mu \mathrm{m}$ along their trajectory in silicon. The amplitude of the signal scales therefore with the length of the particle trajectory in the active silicon of the sensor. A minimum thickness of few $100 \mu \mathrm{m}$ of the sensors was traditionally considered as appropriate for obtaining a good number of electron/hole-pairs. The separation of electrons and holes is done by electric fields as found in the depleted volume of the charge collection diode. Conventional sensor designs extend this depleted zone over the full active sensor volume.

The width $W$ of a reverse biased one-sided abrupt flat junction is given, e.g. in [39], with:

$$
W=\sqrt{\frac{2 \epsilon_{s}\left(V_{b i}-V\right)}{e N_{B}}}
$$

Here, $e$ denotes the elementary charge, $\epsilon_{s}$ the electrical permitivity in silicon, $V_{b i}$ the buildin-voltage of the PN-junction and $N_{B}$ the doping concentration at the lowly doped side of the junction. $V$ is the negative, externally applied reverse biasing voltage of the diod $\AA^{3}$. From this equation, one learns that reaching a $W>100 \mu \mathrm{m}$ requires a combination of lowly doped silicon $\left(N_{B} \lesssim 10^{13} / \mathrm{cm}^{3}\right)$ and high reverse bias voltages of $\sim 100 \mathrm{~V}$. Due to missing commercial interest, this low doping was initially not found in wafers used for CMOS electronics. Moreover, the guaranteed voltage tolerated by CMOS chips does typically not exceed $5 \mathrm{~V}$. Until both limitations vanished thanks to the appearance of optimized CMOS processes (see section 6.4), it was not possible to realize charge collection diodes with thick depleted volume in CMOS technology.

The traditional solution to for handling the limitations consists in realizing the charge collection diode and the amplifiers in two different chips, which are electrically connected hereafter. The diode is placed on a dedicated, very pure and lowly doped wafer, which does not host transistors but may tolerate high voltages instead. The amplifiers are put on a CMOS chip, which is optimized for analogue and digital electronics. This concept allowed for building successful designs like silicon strip detectors or the hybrid pixel detectors used

\footnotetext{
${ }^{3}$ One assumes $N_{B}$ to be negligible w.r.t the doping concentration of the highly doped side. $V_{b i}$ amounts $<1 \mathrm{~V}$ and can thus be neglected in many cases.
} 
e.g. at the $\mathrm{LHC}^{4}$ However, the solution comes with limitations. The material of the sensor chips and the readout chips tends to scatter the charged particles of interest and thus to change their trajectory. Moreover, the necessary interconnections between both chips are expensive, somewhat vulnerable and constrain the size of the sensor cells. Therefore, hybrid pixel and strip detectors are not the first choice, once a physics case requires to measure particle trajectories with highest precision.

CMos monolithic active Pixel Sensors for charged particle tracking (CPS) were developed in order to overcome this limitation. To do so, they integrate the charge collection diode and its amplification chain on one single CMOS chip, which reduces the material and eliminates the interconnections. However, the above mentioned restrictions concerning doping and voltage apply and force a number of compromises in the design. The history of CPS is best understood as a series of increasingly successful trials to bypass and overcome those restrictions. This was among others possible as the CMOS Monolithic Active Pixel Sensors for optical imaging, from which the CPS were initially derived, had a great commercial success, e.g. as cameras of smart phones. This triggered the development of improved CMOS processes, which became also accessible to the particle physics community.

\subsubsection{The classical sensing element}

The classical design of the sensing element of a CPS was designed for being compatible with low resistivity double well CMOS processes relying on wafers with epitaxial layer. A simplified sketch of a CPS implemented in this process is shown in Fig. 5 . The lowest layer of the device is formed from a silicon wafer with a doping of about $p=10^{19} / \mathrm{cm}^{3}$. On top of this wafer, one processes an epitaxial layer, which has a typical doping of few $p=10^{15} / \mathrm{cm}^{3}(\mathrm{P}$ - in the figure) and may show a typical thickness of few $\mu \mathrm{m}$ up to $20 \mu \mathrm{m}$. Above, the designers may add so-called wells $(\mathrm{P}+$ and $\mathrm{N}+$ in the figure), which may have a $\mathrm{p}$ - or $\mathrm{n}$-doping of something like $p=10^{17} / \mathrm{cm}^{3}$. This layer may embed $\mathrm{p}$ - or $\mathrm{n}$-diffusions $(\mathrm{P}++$ and $\mathrm{N}++)$, which show a doping roughly $p=10^{19} / \mathrm{cm}^{3}$. Note that the thickness of the structures as much as the precise doping concentrations vary among different manufacturers. They are, if known at all, subject to non-disclosure contracts.

According to equation 5, a charge collection diode with $N_{B}=3 \times 10^{15} / \mathrm{cm}^{3}$ and $V=5 \mathrm{~V}$ will show $W=1.5 \mu \mathrm{m}$, which is not sufficient to obtain a reasonable signal from an impinging MIP. The limitations of the early CMOS processes in terms of voltage limitation and high doping concentration did not allow for extending this depleted volume. Instead, a typically $\sim 15 \mu \mathrm{m}$ thick epitaxial layer is used as an extension of the diode. This epitaxial layer is not depleted and therefore free of electric fields. The $\sim 1200$ signal electrons generated by a MIP in this layer are therefore not attracted by drift but diffuse to the thin charge collection diodes. This time consuming process succeeds thanks to the $\sim 1 \mathrm{~ms}$ long life-time of the free electrons.

The full sensing element is formed from the epitaxial layer, which is surrounded by the substrate and a p-well layer. The doping gradients between the lowly doped epitaxial layer and each of the highly doped p-well and substrate generate a small potential drop of $\sim 100 \mathrm{mV}$,

\footnotetext{
${ }^{4}$ See e.g. [22] for a comprehensive introduction.
} 


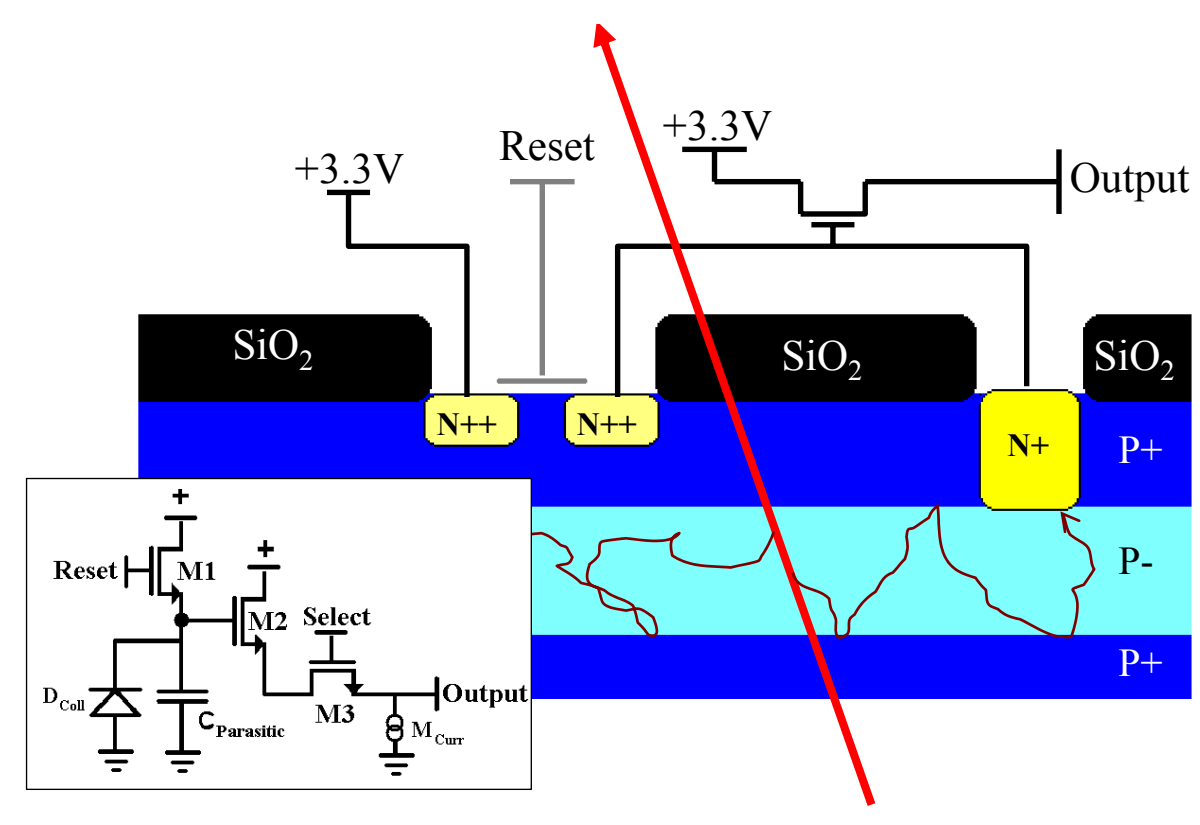

Figure 5: Fundamental layout of a CMOS-sensor. The trajectory of an impinging minimum ionizing particle and the diffusion paths of two free electrons is shown. The diagram of the preamplifier shown is displayed in the lower left box.

which hampers the electrons from leaving the epitaxial layer ${ }^{5}$. The photo-diode is formed from n-well implantations, which penetrate the p-well layer, and the epitaxial layer.

Despite the charge is collected by diffusion, non-irradiated classical CPS pixels may show a rather high charge collection efficiency of fairly above $90 \%$. However, the diffusion creates a significant charge sharing and only about $25 \%$ of the electrons are found in the diode located closest to the impact point of the impinging particle (seed pixel). Most of the remaining signal charge is spread over a cluster of $3 \times 3$ pixels while few electrons recombine before reaching a pixel diode and are lost.

The sensing elements of CPS provides therefore a rather tiny signal charge of typically some 100 electrons. To distinguish this signal from noise, one requires for a very low noise pre-amplifier, which must moreover be suited for being integrated into the individual pixel.

\subsubsection{The 3T-amplifier}

In the standard double-well CMOS processes available in the early days of CPS development, the on-pixel amplifiers had to rely exclusively on NMOS transistors. This is as building a PMOS (p-n-p) transistor requires the use of a n-well implantation. This n-well combines with the epitaxial layer to an unwanted second charge collection diode. This diode competes with the primary charge collection diode in terms of charge collection and such reduces the signal sensed by the latter diode in a rather dramatic way. Still, PMOS transistors could be placed on the same chip in non-sensitive areas aside the pixel matrix. This limitation vanished in

\footnotetext{
${ }^{5}$ See Sec. 6.1 .3 .4 for further information.
} 
modern CMOS processes as the $\mathrm{n}$-well of the PMOS transistor and the active volume may be separated by a deep p-well implantation. However, this feature was not available for the design of the first CPS generations, which limited the design options substantially. This is as building blocks like high gain amplifiers and discriminators require PMOS transistors and can thus not be used in the on-pixel electronics.

The on-pixel amplifier of the traditional 3T-pixel forms a rather successful solution to overcome this limitation. The diagram of the charge-to-voltage amplifier is shown in the box of Fig. 5. It is formed by three transistors, the parasitic capacitance of the pixel diode and a current source, which is typically located outside the sensor-chip. The amplifying process is twofold: First, a charge-to-voltage conversion is performed by charging the collected electrons into the tiny capacitance and generating such a voltage drop seen by the gate of the transistor M2. This transistor and the current source $M_{\text {curr }}$ form a source follower with a voltage gain slightly below one. The latter buffers the signal and such reduces the output impedance 6 of the pixel. The output signal $U_{S}$ of the 3T-pixel is given with:

$$
U_{S}=g \cdot \frac{Q_{s}}{C}
$$

Here, $Q_{S}$ is the signal charge, $C \approx 10 \mathrm{fF}$ the capacitance of the pixel and $g$ the summed gain of the source follower and possible further voltage amplification stages, which are located outside the pixel.

For non-irradiated pixels, the noise of the pixel is dominated by the amplification chain. The signal-to-noise ratio can be increased by reducing $C$ while increasing $g$ by means of adding voltage amplifiers located outside the pixel amplifies both, the signal and the noise. Reducing $C$ suggests to reduce the size of the diode and the transistor gate of M2. This strategy has to be balanced against the bad charge collection capabilities of tiny collection diodes and the occurrence of Random Telegraph Signal (see Sec. 8.1.1) in transistors with too small gates. The typical noise of a good and non-irradiated 3T-pixel amounts $\sim 10$ e ENC (equivalent noise charge).

Besides the signal charge, the leakage current of the collection diodes discharges the pixel capacitance. To avoid a saturation of the pixel, this capacitance is recharged by opening the reset switch M1. The recharging is process somewhat history dependent [40] and, due to kTC-noise, not very accurate. A substantial improvement in the noise performance of the pixel can be achieved by means of correlated double sampling (CDS), which consists in taking a reference for the voltage $U_{1}\left(t_{1}\right)$ of the pixel before starting a particle measurement but after performing the reset cycle. After the integration time of this particle measurement is completed, a sample $U\left(t_{2}\right)$ is taken and the voltage drop in the pixel is derived by subtracting

\footnotetext{
${ }^{6}$ The output impedance of a signal source is a measure for the amount of current, the source may deliver without showing a voltage drop. This is modelled in a most simple picture by a resistor/impedance, which is put into series to an ideal voltage source. As a good rule of thumb, the output impedance of a signal source should remain by one order of magnitude below the input impedance of the next amplification stage.

The capacitance of a CPS pixel stores only few 100 electrons signal charge, which does not allow to deliver any current and thus shows a very high output impedance. The source follower does however not consume current from its input (very high input impedance) and delivers a macroscopic current at its output (low output impedance). This does not amplify the voltage signal but stabilizes it to the level needed to drive the voltage amplifiers of the next amplification stage. This reduction of the impedance is denoted as buffering.
} 
$U_{S}=U\left(t_{2}\right)-U\left(t_{1}\right)$. This eliminates the signal offset introduced by the reset noise. However, the reset of the pixel and the reference measurement introduce a dead time.

The leakage current of the diode can be considered as constant in time. It thus provides a DC-pedestal of $U_{S}$, which can be measured and subtracted. The capability of 3T-pixels to indicate the leakage current of its diode down to a fraction of fA makes those pixels a valuable tool for understanding radiation damage in the active volume and the pixel diode.

\subsubsection{The rolling-shutter readout scheme}

Experiments of sub-nuclear physics aim usually to reach high counting rates in order to provide the statistics needed to observe rare particles and subtle effects. To match this goal, one would preferably connect each pixel with an individual, fast readout chain, which delivers the pixel signal immediately to the outside world. This is however not feasible as the number of electrical connections between the chip and the outside world is restricted to $\sim 100$ while a full size CPS hosts typically $\sim 10^{6}$ pixels. The simple solution used in first generation CPS consists in connecting all pixels consecutively to an analogue output line (multiplexing) and to process the data stream obtained with external resources like an external ADC. To connect and to disconnect the pixel from this common readout line is the mission of the "select" switch of the 3T-pixel (transistor M3 in Fig. 5. In this readout scheme, the beginning $\left(t_{1}(i)\right)$ and the end $\left(t_{2}(i)\right)$ of the integration time of each pixel is given with:

$$
\begin{aligned}
& t_{1}(i)=t_{0}+i / f \\
& t_{2}(i)=t_{1}(i)+N / f
\end{aligned}
$$

Here, $f$ stands for the readout frequency of the readout line, $N$ for the number of pixels connected to this line and $t_{0}$ for time of the readout of the first pixel. The integration time of the detector is given with:

$$
t_{\text {int }}=N / f
$$

As the frequency of the readout $f$ is restricted to $\sim 50 \mathrm{MHz}$ for an analogue readout, the time resolution of this kind of readout remains at $\sim 10 \mathrm{~ms}$. The readout may be accelerated by using a couple of readout lines in parallel, which is however limited in practice by the number of external channels (e.g. high speed ADCs) required.

\subsection{CPS with accelerated time resolution and rate capability}

\subsubsection{Design challenges and approaches for solutions}

The time resolution and the rate capability of the 3T-pixel is limited by two major issues. On one hand, the pixel reset and the need to obtain a reference frame before initiating the particle measurement generates a significant dead time, which is not acceptable for modern particle physics experiments. Moreover, the speed of the over-all system is limited by the data bus transporting the signals obtained from the pixel to the outside world.

The first issue was solved by replacing the reset switch by a system providing a continuous leakage current compensation. Thanks to this, the reset cycle became obsolete and the related dead time was eliminated. This feature is implemented in pixel structures, which are denoted 

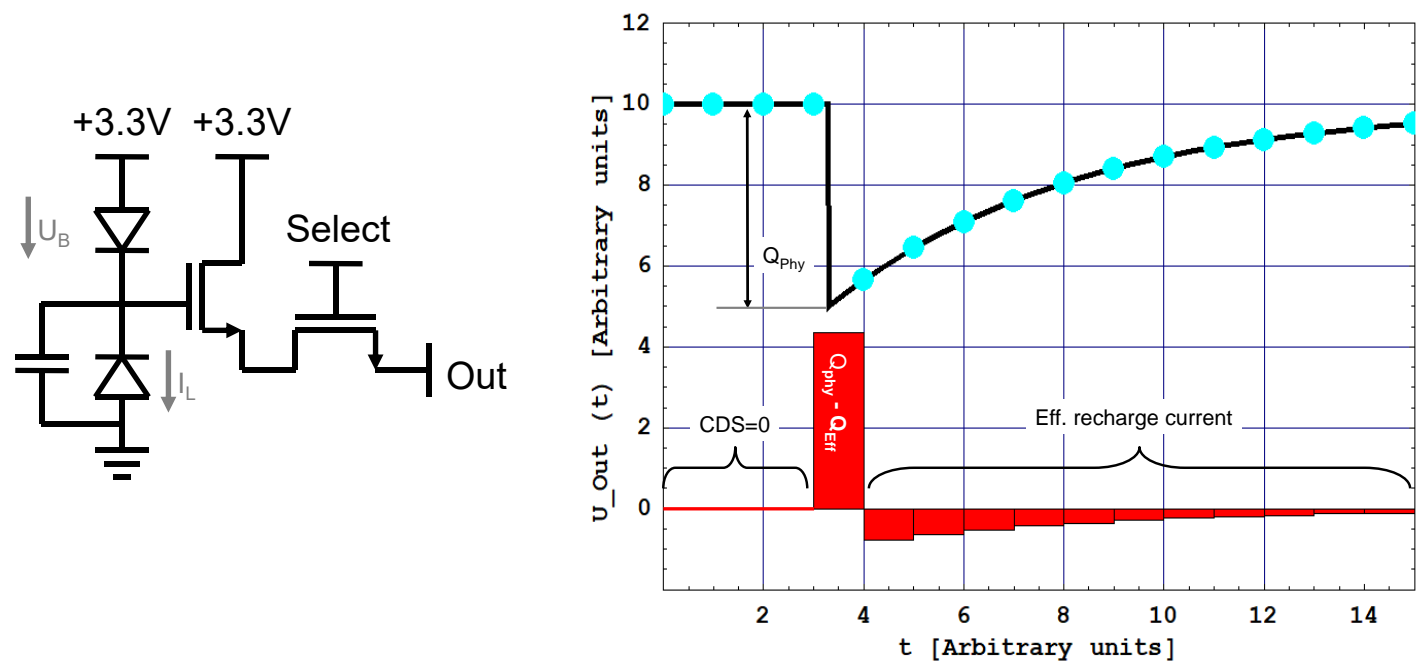

Figure 6: Left: Diagram of a SB-pixel. Right: Response of a SB-pixel to a particle signal. The signal $Q_{p h y}$ is cleared slowly after the hit impinges. Performing CDS shapes the signal.

as Self-Bias (SB-)pixels. The second issue was addressed by digitizing the data if possible already on the pixel, to compress it on the chip and to submit only compressed digital data to the outside world. The compression is in simple terms done by exclusively submitting the address of the few firing pixels instead of charge information of all pixels, which reduces the data volume of frames with low occupancy dramatically.

Realizing this concept is complicated by the fact that the analysis of CPS data includes a number of steps like pedestal correction and correlated double sampling, which were initially done offline and had to be integrated into the ASIC. Copying the traditional digital processing came out as unrealistic as the memory required could not be placed to the sensors. Instead, the functionalities had to be realized with analogue electronic circuits. Designing circuits doing the analogue processing of few 100 electron signals in CMOS technology came out to form a tough engineering challenge. This is as the individual CMOS components show significant production tolerances, which generate offsets in the dark signal of the devices. Compensating those offsets e.g. by means of trim DAQs into highly granular pixels is excluded by space constraints. Therefore, all amplification and discrimination circuits have to be designed with internal analogue offset compensation.

\subsubsection{Eliminating the dead-time - The Self-Bias-Pixel}

In the self-bias pixel (SB-pixel, see Fig. 6, left), the reset transistor is replaced by a highohmic, forward biased diode. The current passing through this diode replaces continuously the charge, which was lost to the leakage current of the collection diode. Therefore, the reset cycle and the related dead time become obsolete. Moreover, the pedestals of the pixels disappear after performing CDS (see Fig. 6, right). In case the pixel is hit, the signal voltage $U_{S}$ increases the voltage drop at the biasing diode and the current passing this diode increases. This increase removes the signal charge from the pixel capacitance. This clearing process can 
be well modelled with an exponential decay of the signal charge:

$$
Q_{S}(t)=Q_{P h y} \cdot \exp \left(-\frac{t}{\tau}\right)
$$

Here, $Q_{P h y}$ denotes the signal charge created by the particle impact at $t=0$ and $\tau$ the time constant of the clearing. This constant should be kept as long as feasible by design. It decreases dynamically in case a fast series of hits creates a sizeable signal charge in the pixel capacitance. Therefore, SB-pixels don't saturate in practical terms and the pixel remains sensitive to further hits during the clearing process. However, the charge injected by the clearing reduces the amplitude of the consecutive particle hit. Moreover, in the case of high occupancies and/or high leakage currents, $\tau$ approaches the integration time of the pixel and a significant fraction of $Q_{S}$ is cleared before the pixel is read out. This and specific radiation damage effects will be discussed in more detail in Sec. 5.1.2.

\subsubsection{From sequential to column parallel - The clamping-pixel}

While the SB-pixel features a leakage current compensation, it is not yet suited to perform CDS and signal discrimination. Those building blocks were added in the clamping pixel (CPpixel) [42]. The CP-pixel is designed for standard CMOS technology, which prohibits the use of PMOS transistors and thus discriminators in the pixel area. Instead, the discriminators are placed on the same chip aside the pixel matrix.

The readout is typically organized in a column parallel way, which means that the signal of all pixels of a column is multiplexed to one common discriminator. This discriminator supports typically a pixel readout frequency of $\sim 5 \mathrm{MHz}$ and is thus one order of magnitude slower than the related external devices. However, as about 1000 readout blocks are operated in parallel, one obtains a speed-up of two orders of magnitude with respect to the traditional readout.

A simplified diagram of a CP-pixel and a switching diagram $7^{7}$ of a pixel readout is shown in Fig. 7. The sensing element may be similar to the one of a 3T- or SB-pixel and shares the related properties. The source follower transistor is replaced by a AC or DC coupled voltage amplifier. This amplifier sends its signal via a second capacitor to the clamping node $N_{2}$. The potential of the latter is fixed prior to each readout cycle by opening the $R s t$ switch. Hereafter, it is buffered by a source follower and forwarded via the Calib switch to an analogue storage capacitor located in the end-of-column discriminator block. After the integration time, the potential is sent via the Read switch to the second capacitor and the potential of both capacitors is compared by the comparator. Thanks to the AC-coupling between the amplifier and the node $N_{2}$, the difference obtained is equivalent to the signal after CDS. If this signal exceeds a threshold, a digital hit indication is sent to the output.

The clamping pixel was found decisively less vulnerable to the production tolerances of the CMOS process than earlier design attempts [41, which aimed to integrate the storage capacitors and differential amplifiers needed for doing CDS into the individual pixels. In good designs, the offsets caused by those tolerances remained substantially below the thermal noise

\footnotetext{
${ }^{7}$ Note that the diagram starts at the end of a pixel integration time and ends at the beginning of the consecutive integration time.
} 


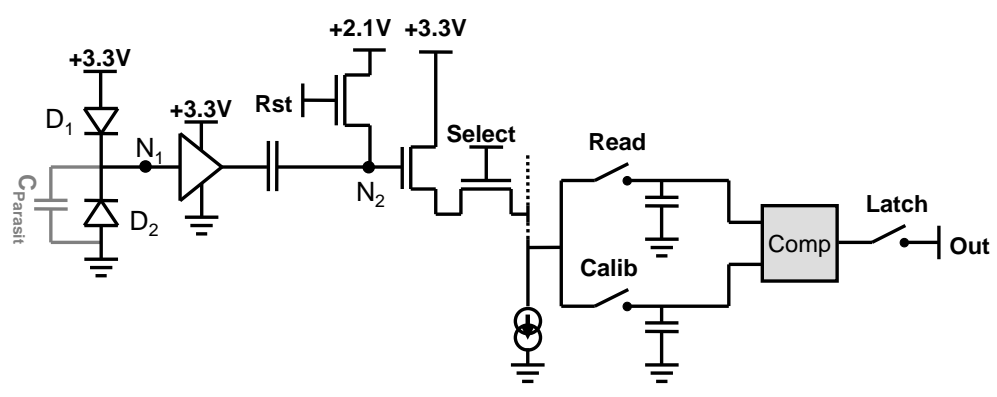

Figure 7: Diagram of the DC coupled SBversion of a clamping pixel and its readout sequence. In the 3Tversion, the diode $\mathrm{D}_{1}$ is replaced by a reset transistor, in the ACcoupled version, a capacitor is added at the point $\mathrm{N}_{1}$. After [42, 43].

of the pixels. After adding a digital data compression stage, the architecture was successfully used by multiple users.

\subsection{Performances of selected CMOS sensors based on standard CMOS processes}

Classical CPS designed for charged particle tracking showed a surprisingly good performance from the start. The first first prototypes of the MIMOSA-series demonstrated a single point resolution of $1-2 \mu \mathrm{m}$ and a detection efficiency close to $100 \%[3$ in $\mathrm{a} \sim 100 \mathrm{GeV} / \mathrm{c}$ pion beam at the CERN-SPS. Those excellent results were soon reproduced with a full size CPS (MIMOSA-5) with up to $10^{6}$ pixels on active areas as large as $4 \mathrm{~cm}^{2}$ [3, 4]. Moreover, it became clear that the devices can be thinned to $50 \mu \mathrm{m}$ without loss of performance. The charge sharing of the pixels allowed to apply centre-of-charge methods, which improved the spatial resolution to $\sim p / 10$, where $p$ is the pixel pitch [44]. This has to be compared with a value of $p / \sqrt{12}$, which holds for pixels showing no charge sharing.

The properties of selected detectors of the MIMOSA-series 8 is shown in Tab. 1. The first sensor listed, MIMOSA-5 was mostly a technology demonstrator, which relied on 3T-pixels. It was not really used for measurements due to its long integration time and the dead-time required for resetting the device. Instead, a prototypes relying on SB-pixels were designed. This R\&D helped a lot to improved our understanding on the radiation hardness of CPS and yielded into sensors for the early EUDET-telescope (MimoTEL) [45. However, the research line was abandoned as fully integrated sensors with clamping pixels became available.

The first of those sensors, MIMOSA-26 [46, 47] was a fully integrated detector featuring column parallel readout, internal data discrimination, internal data sparsification and could

\footnotetext{
6.4

${ }^{8}$ The properties of other sensors, which typically rely on advanced CMOS processes, are discussed in Sec.
} 


\begin{tabular}{|c|c|c|c|c|}
\hline Sensor & MIMOSA-5 & MIMOSA-26 & $\begin{array}{l}\text { MIMOSA-28 } \\
\text { (ULTIMATE) }\end{array}$ & FSBB-M0 \\
\hline Year & 2001 & 2008 & 2011 & 2015 \\
\hline Technology & AMS $0.6 \mu \mathrm{m}$ & AMS $0.35 \mu \mathrm{m}$ & AMS $0.35 \mu \mathrm{m}$ & Tower $180 \mathrm{~nm}$ \\
\hline Epitaxial layer & Low res. & High res. & High res. & High res. \\
\hline User & None & $\begin{array}{c}\text { EUDET } \\
\text { NA61/SHINE }\end{array}$ & STAR & None \\
\hline Pixel & $1024 \times 1024$ & $1152 \times 576$ & $960 \times 928$ & $416 \times 416^{a}$ \\
\hline Pixel size & $17 \times 17 \mu \mathrm{m}^{2}$ & $18.4 \times 18.4 \mu \mathrm{m}^{2}$ & $20.7 \times 20.7 \mu \mathrm{m}^{2}$ & $22 \times 33 \mu \mathrm{m}^{2}$ \\
\hline Pixel type & $3 \mathrm{~T}$ & Clamping & Clamping & Clamping \\
\hline $\begin{array}{l}\text { Spatial resolution } \\
\text { Readout time }\end{array}$ & $\begin{array}{l}\sim 2 \mu \mathrm{m} \\
6.5 \mathrm{~ms}\end{array}$ & $\begin{array}{c}3.5 \mu \mathrm{m} \\
115.2 \mu \mathrm{s}\end{array}$ & $\begin{array}{c}3.8 \mu \mathrm{m} \\
185.6 \mu \mathrm{s}\end{array}$ & $\begin{array}{c}\sim 4.5 \mu \mathrm{m} \\
41.6 \mu \mathrm{s}\end{array}$ \\
\hline Discriminator & External ADC & End of column & End of column & End of column \\
\hline Readout parallelism & 4 pixel (analogue) & Single line & Single line & Double line \\
\hline Power dissipation & $\mathrm{N} / \mathrm{A}$ & $250 \mathrm{~mW} / \mathrm{cm}^{2}$ & $160 \mathrm{~mW} / \mathrm{cm}^{2}$ & $<160 \mathrm{~mW} / \mathrm{cm}^{2}$ \\
\hline Max. data rate & $4 \times 40 \mathrm{MHz}^{\mathrm{a}}$ & $160 \mathrm{Mbps}$ & $320 \mathrm{Mbps}$ & $2 \times 320 \mathrm{Mbps}$ \\
\hline $\begin{array}{l}\text { Ionizinig rad. tol. } \\
\text { Non. Io. rad. tol. }\end{array}$ & $\begin{array}{c}\sim 100 \mathrm{krad} \\
<10^{12} \mathrm{n}_{\mathrm{eq}} / \mathrm{cm}^{2}\end{array}$ & $\begin{array}{c}150 \mathrm{krad} \\
>10^{13} \mathrm{n}_{\mathrm{eq}} / \mathrm{cm}^{2}\end{array}$ & $\begin{array}{c}150 \mathrm{krad} \\
>10^{13} \mathrm{n}_{\mathrm{eq}} / \mathrm{cm}^{2}\end{array}$ & $\begin{array}{c}>1600 \mathrm{krad} \\
>10^{13} \mathrm{n}_{\mathrm{eq}} / \mathrm{cm}^{2}\end{array}$ \\
\hline
\end{tabular}

${ }^{a}$ Three FSBB were intended to form a full reticle size sensor.

Table 1: Performances of reticle size sensors relying on standard CMOS processes (MIMOSAseries) and partially depleted pixel diodes.

be configured by a JTAG interface. The readout is binary but the spatial resolution is better than $p / \sqrt{12}$ as the charge sharing between the pixels turns into different cluster shapes, which may be analysed by means of centre-of-gravity methods. The sensor was available in a version with standard epitaxial layer but also in version with more radiation tolerant high resistivity epitaxial layer. The sensor is being used for the present EUDET-telescope. Moreover, it was used for the prototype of the CBM-Micro Vertex Detector [9, 10] and the first generation (so called small acceptance) vertex detector of the NA61/SHINE experiment [14]. The MIMOSA28 (ULTIMATE) [48] sensor is a larger and slightly slower device, which essentially relies on the same architecture of MIMOSA-26. It can be considered as the most prominent sensor as it was used in the STAR-PiXeL detector [49]. The FSBB-M0 was the most developed sensor relying on clamping pixels. It was developed aiming for a use in the ALICE inner tracker and was optimized for fast readout, among others by reading out two lines in parallel. The sensor relied already on a next generation process (Tower 180nm) but did not use specific features of this process. However, the novel process allowed for a substantially improved tolerance to ionizing radiation. 


\section{Methods for observing radiation damage}

In the most simple approach, the radiation tolerance of a device is evaluated by measuring its properties before and after irradiating it. As any radiation dose creates microscopic radiation damage, one expects the properties to change during irradiation. However, the related modification may remain without impact on the macroscopic properties of the device. A device is considered as tolerant to a specific radiation dose, if it still operates according to its specifications after irradiation. Typical mistakes in radiation tolerance studies consist in not defining the specification to be fulfilled in a clear and comprehensive way. Moreover, the very human wish to observe a high radiation tolerance may turn into confusing a missing instrumental sensitivity to radiation damage with the absence of this damage.

Besides estimating the radiation tolerance of a given device, there is an interest in spotting the damaging mechanism limiting this tolerance. The latter allows to fix the weak points of the design. Those kind of studies are particularly complex in highly integrated devices like CPS. This is because the sensing elements of CPS can only be accessed via the internal amplification chain? which complicates the measurements and limits the number of accessible parameters. Consequently, studies on radiation tolerance are preferably carried out with sensors with a simple readout chain, e.g. with 3T- and SB-pixels. A brief overview over the accessible parameters for those pixels will be given in the following. More detailed information can be found e.g. in [50].

\subsection{Setting up the testing hardware}

As CPS are sensitive to light and many radiation effects are temperature sensitive, they should be tested in a temperature controlled dark chamber. The dark chamber should be air tight to avoid a destruction of the sensor by condensed water. Unless dry nitrogen is injected, the sensors should be started at room temperature and cooled only while running. Few tens of minutes are typically required to reach a stable thermal equilibrium.

In 3T-pixels and SB-pixels, the readout of the pixel properties is typically done via a linear, analogue amplification chain. The signal is hereafter digitized by means of an ADC. Due to the relatively wide spread of the dark signal of the pixels as compared to the pixel noise, this ADC should provide at least a 12-bit resolution. After applying CDS, the pixel amplitude may be indicated negative. The readout chain has thus to support negative numbers. Besides of showing a wide spread from pixel to pixel, the dark signal of the pixels changes significantly with temperature. Caution is required to keep this signal in the linear range of the readout chain as saturated pixels may generate rather funny electronic effects. The symmetry of the charge of the signal clusters is worth checking as an asymmetry in the average charge of all pixels in the order of readout is an indicator for an issue in the analogue readout chain.

\footnotetext{
${ }^{9}$ One may rightfully argue that it is possible to build isolated sensing elements and to connect them with external high precision instruments. However, the capacities and leakage currents of CMOS pixel diodes are tiny and typically below the sensitivity of conventional external instruments. Moreover, trials to follow this approach with groups of diodes revealed that isolated charge collection diodes and charge collection diodes connected to a pixel may show different responses to radiation damage (see Sec. 5.1.1.1).
} 


\subsection{Irradiating the sensors}

A good choice of irradiation sources may ease spotting the origin of radiation damage. Most charged particles generate ionizing and non-ionizing radiation at the same time and generate therefore both, bulk and surface damage. However, X-rays with $\sim 10 \mathrm{keV}$ have too small momentum to displace atoms and to create bulk damage. Neutrons are missing the charge needed to ionize electrons and generate therefore almost exlusively bulk damage. Using $10 \mathrm{keV} \mathrm{X}$-rays and $\sim 1 \mathrm{MeV}$ neutrons does thus allow to separate the effects of bulk and surface damage. The use of charged hadron beams should be considered whenever a specific effect is suspected to vary with the relative amount of point defects and defect clusters in the bulk of the silicon.

\subsubsection{Irradiating CPS with X-rays}

Irradiations with X-rays are typically done with medical X-ray tubes, which are complemented with a dosimetry system. The typical dose rates of $\gtrsim 100 \mathrm{krad} / \mathrm{h}$ allow for fast irradiations but let room for potential dose rate dependent effects. The device under irradiation has to be biased and operated during irradiation to provide the fields required for separating the electron/hole pairs in the $\mathrm{SiO}_{2}$ structures. Not biasing is equivalent to reducing the dose by a significant factor (see [21] for data on the impact of biasing voltages to surface damage). The biasing is best done by mounting the device on a test PCB and operating it. Obviously, it is of advantage to test the device before irradiation. Note that the active components of the PCB are typically not radiation tolerant and have to be shielded against the X-rays, e.g. with $\gtrsim 1 \mathrm{~cm}$ brass.

As a part of the surface damage is reduced by room temperature annealing, the dose rate and the temperature during irradiation [51] may have an impact on the results. To avoid unwanted/uncontrolled annealing, the irradiated device should be tested already during or short after the irradiation. Moreover, one should avoid to expose the sensors to high temperatures. This is of particular concern if the irradiated device has to be transported prior to testing it.

\subsubsection{Irradiating CPS with neutrons}

Neutron irradiations are typically performed with direct reactor neutron beams provided by suited research reactors. In case the neutron beam contains a sizeable amount of additional thermal neutrons, one should consider that P-doped CPS might receive additional damage via the neutron capture reaction ${ }^{10} \mathrm{~B}(\mathrm{n}, \alpha){ }^{7} \mathrm{Li}$ (see Sec. 8.2).

Unlike CPS, test PCBs may be activated by neutrons and should thus not be used for neutron irradiation. Consequently, the chips cannot be powered during irradiation. This is beneficial. It reduces the unwanted surface damage caused by the $\gamma$-ray background of the neutron beam. However, the sensors cannot be tested before or during irradiation. Instead, one compares the properties of irradiated sensors with non-irradiated devices of the same series. This imposes uncertainties due to production tolerances.

The CPS may be wrapped in aluminum or held by Gelpacks during irradiation. The latter were found neither activated nor degraded by doses of $10^{14} \mathrm{n} / \mathrm{cm}^{2}$ (lower limit). However, it 


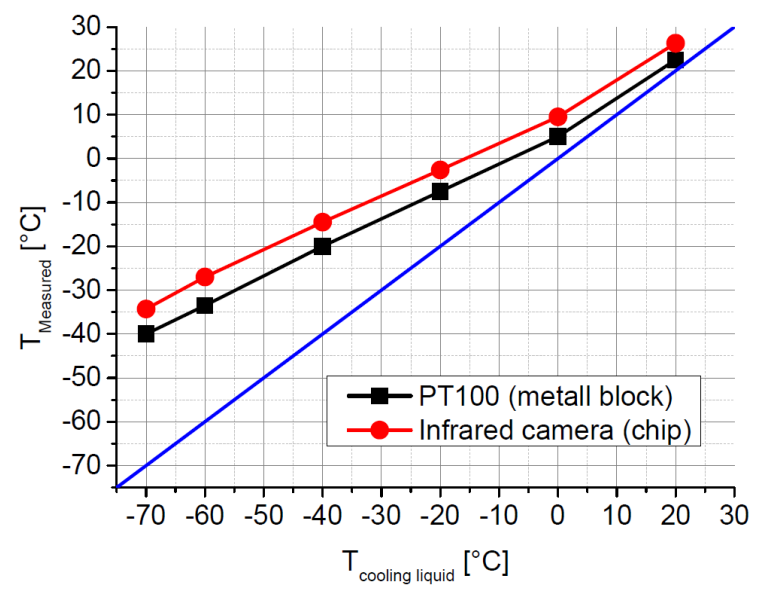

Figure 8: Temperature of a MIMOSA-18 sensor as measured with an IR camera in comparison to the temperature of the supporting metal block as a function of the coolant temperature of the chiller. See text. From [52].

is mostly impossible to remove $50 \mu \mathrm{m}$ thin CPS from the packs. Therefore, thick chips should be chosen. Due to limited production yields, some of the non-tested sensors may be broken already before irradiation. One should thus irradiate several sensors per dose level in order to obtain at least one (initially) working device.

Non-ionizing radiation damage in CPS was found to show less room temperature annealing than ionizing radiation damage. However, few days were typical required for placing and bonding the sensors on PCB, which lets room for overlooking such effects.

\subsection{Accessible quantities and their measurement}

\subsubsection{Chip temperature}

Measuring and controlling the temperature of the chip appears simple but comes out to be tricky as the chip is self-heating during operation and as the heat contact via the supporting PCB toward the water cooled cooling support is far from perfect. Moreover, it is hard to install a temperature sensor on a CPS prototype without endangering the bonds. The use of IR-thermometers is hampered by the high reflectivity of CMOS chips and indicates the arrangement and density of the metal lines of the chip rather than its temperature [53]. This issue was solved by spraying a MIMOSA-18 sensor with non-conductive, black colour (TETENAL 105202 camera varnish) [52]. Figure 8 shows the temperature measured for this chip in comparison with the coolant temperature of the cooling system and the indication of a PT100 sensor placed nearby the sensor PCB on the cooling support. The plot can be considered as representative for most MIMOSA sensors relying on serial analogue readout. It should be mentioned that, in the absence of better options, coolant temperature was used as a temperature reference in a couple of early publications on the radiation tolerance of MIMOSA series CPS. As the IR-measurement is too complex for a daily use, later works indicate the reading of the PT100 sensor.

\subsubsection{Leakage currents}

The leakage current of the charge collection diodes of CPS is typically too small for a direct measurement by means of an ammeter. The procedure may only be applied with large groups 
of diodes connected in parallel, which increases the current proportional to the number of diodes. Doing this with isolated diodes, which are not surrounded by the remaining pixel structures, was found non-reliable. A mismatch of a factor 20 was observed when comparing the directly measured leakage current of isolated charge collection diodes with the one of their counter parts, which were integrated in a pixel (see [40], P. 213). Despite the origin of the effect has never been clarified in detail, it is recommended to use the indirect measurement approach introduced below. Still, direct measurements may be used to estimate the leakage current of SB-pixels. This is feasible provided that the biasing of the pixel diodes is separated from the general bias lines of the chip.

The suggested measurement procedure for leakage currents consists in extracting the leakage current of the pixel diodes from the dark charge being indicated by 3T-pixels after CDS processing. Knowing the gain of the pixels from measurements with a ${ }^{55} \mathrm{Fe}-$ source (see below), this dark signal can be translated from voltage to charge in units of electrons. The current is computed accounting for the known integration time of the pixel. In order to reduce the impact of the noise, one does typically take $\sim 100$ samples and computes the mean value. Note that the leakage current indicated by this kind of measurement increases during a few seconds long in the warm-up phase of the sensor. It is measured for the the individual pixels and subject to significant production tolerances of their diodes. The result is typically displayed as the mean leakage current of all pixels. The width of the typically asymmetric distribution is indicated as error bars, which should not be confused with the substantially lower uncertainty of the mean value measurement. The leakage current measurement of the diode may be biased by i) the drain-to-source leakage current and ii) the bulk-to-source leakage current of the reset transistor.

\subsubsection{Pixel noise (analogue readout)}

CPS exhibit multiple kinds of noise, which are caused by the noise of their intrinsic components (high frequency noise), pick-up from external sources (common mode noise) and Random Telegraph Signal (RTS, see Sec. 8.1.1. Moreover, the pixel-to-pixel fluctuation of the dark signal of the pixels, despite technically not a noise, is sometimes referred to as fixed pattern noise. It is of relevance in case the signal discrimination thresholds cannot be tuned for the individual pixels. The different noise components may be measured as follows:

4.3.3.1 High frequency noise The measurement of high frequency noise is done in analogy to the measurement of the leakage currents of the pixels. Instead of the mean value of multiple pixel signals after CDS, the related standard deviation is measured. As for the leakage current, the high frequency noise varies from pixel to pixel and the variation follows a rather broad distribution, which is often expressed in the error bars of the related measurements. Note that a low high frequency noise is not necessarily turning into a low dark rate as the latter is often determined by hot pixels being affected by RTS. This consideration is of particular importance as strategies for reducing the capacitive, average noise of the pixels tend to increase the number and noise of hot RTS-pixels (and vice versa). Besides of RTS, the noise measurement may be biased by the noise of the external readout chain including the discretization noise of the ADC and common mode noise. 
4.3.3.2 Common mode noise For 3T- and SB-pixels with serial analogue readout, a low frequency pick up (e.g. $50 \mathrm{~Hz}$ from the electric grid) manifests itself as a slow modulation of the pixel signal as function of the pixel number in the order or the readout. To some extend, the pixel dark signal (before and after CDS) samples the pick up somewhat similar to an oscilloscope, where the $\mathrm{y}$-axis is the amplitude of the pick up and the $\mathrm{x}$-axis corresponds to the pixel number and thus to the time of sampling. This width of the signal is determined by relation between the frequency of the noise and the frequency of the readout clock. As the pick up injects a somewhat similar signal into multiple pixels, it is often referred to as common mode noise.

The common mode noise may be estimated by computing the average pixel signal after CDS of a group of neighbouring pixels (in the sense of readout) within one frame. A filtering of the noise can be done in software by subtracting this average from the signal of the pixels in the group. A significant common mode noise points to a missing filtering of sensitive biasing lines and may be reduced in hardware by adding a low pass (e.g. a capacitor) to this line.

In pixels with digital rolling shutter readout, common mode noise manifests itself as groups of firing, consecutive pixels or lines. This effect can only be alleviated by hardware improvement. Note that under bad circumstances, CPS generate common mode by themselves. This was observed for chips with integrated data sparsification circuits, which were biased with long flex print cables. Once the sparsification circuit obtained a high data load, its current consumption increased and the resistance in the mass bias line modified the common digital and analogue mass potential. This indirectly increased the threshold, which reduced the data load. As an outcome, the system started to oscillate.

4.3.3.3 Random Telegraph Signal (RTS) The term RTS reflects the observation that the current passing diodes and MOSFETs may be modulated between different discrete states . To observe this effect, the dark signal of the individual pixel has to be sampled for up to several minutes and to be plotted as the function of time. Sampling the signal after CDS is particularly sensitive to RTS in the sensing diode of 3T-pixels, sampling the raw pixel signal may exhibit RTS in the input stage of the on-pixel amplifyier (typically the source-follower transistor). More details on RTS are found in Sec. 8.1.1.

4.3.3.4 Fixed pattern noise The term fixed pattern noise describes the spread of the dark signal of multiple pixels and is thus technically not a noise. This spread is rather excessive in CPS but is reliably eliminated by CDS processing for pixels with analogue output. For pixels with in-pixel CDS circuits, the quantity forms an important performance parameter and may be extracted from the spread of the discrimination thresholds of the individual pixels by means of standard S-courve measurements.

\subsubsection{The ${ }^{55} \mathrm{Fe}$ amplitude spectrum - gain and charge collection efficiency}

The term charge collection efficiency (CCE) denotes the number of signal charge carriers collected as compared to the number of signal charge carriers created in the active medium of the sensor. In CPS, signal charge carriers may escape from the charge collection diode of the pixel penetrated by a particle by i) recombination and ii) by the diffusion of charge carriers 


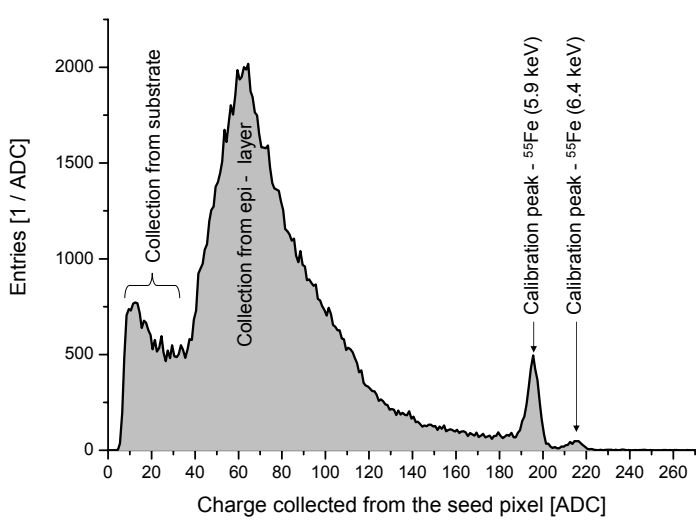

(a) Signal from the seed-pixel.

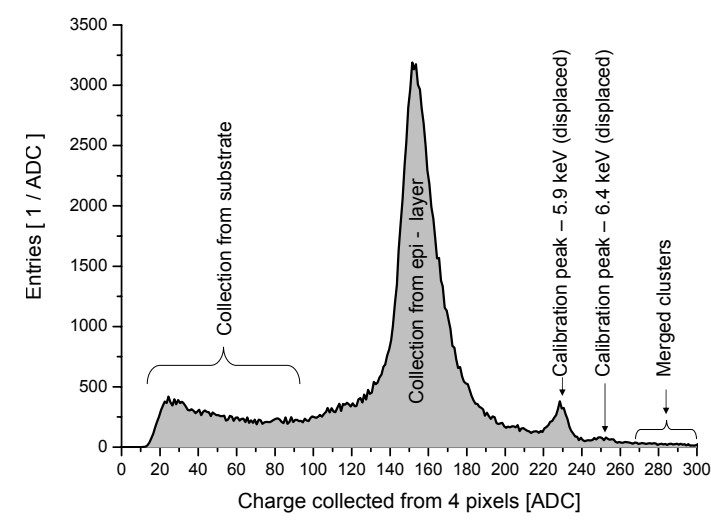

(b) Summed signal of the 4 pixels with highest signal of a signal cluster. Summing the signal of all pixels would move the main "collection" peak to the "calibration" peak.

Figure 9: Typical amplitude spectrum as recorded by illuminating a CPS with low resistivity epitaxial layer with photons from a ${ }^{55} \mathrm{Fe}$-source.

towards neighbouring pixels. The second effect is typically dominant and the full charge is only seen if the signal of all pixels of a pixel cluster is added. Any statement on the related $\mathrm{CCE}$ is only meaningful if the number of pixels used for building this sum is stated.

The CCE of CPS is measured by injecting a known number of signal charge carriers by means of a monochromatic X-ray source. The most suited source is ${ }^{55} \mathrm{Fe}$, which emits dominantly $5.9 \mathrm{keV}$ photons and thus injects 1640 signal electrons. The related hits are recorded and the signal amplitudes are histogrammed to an amplitude spectrum, which is sometimes also called charge collection spectrum. A textbook example of such spectra is shown in Fig. 9. The x-axis of those spectra represents the amplitude of a signal as recorded by a pixel. The $\mathrm{y}$-axis defines, how often this amplitude was recorded. It is instructive to assume that the number of X-ray interactions in a sub-volume of the active medium of the pixel does not depend on the location of this sub-volum 10 . If this assumption of a uniform illumination is made, one may interpret the spectrum as a measure of the volume (y-axis) providing a certain charge collection efficiency ( $\mathrm{x}$-axis).

The spectrum in Fig. 9a represents the amplitudes recorded by the central, so-called seed pixel of a pixel cluster. This pixel collects by definition the highest fraction of the signal charge. The spectra was taken with a sensor relying on 3T-pixels and a low resistivity epitaxial layer. Only a small fraction of the active volume is depleted and most charge is collected by thermal diffusion. The spectrum of such sensors shows two distinguished peaks, the so-called "charge collection peak" and the so-called "calibration peak". Those peaks are generated by two major sub-volumes of the active medium, which show each an about uniform charge collection efficiency (CCE). The "collection peak" is associated with a charge collection from the non-depleted epitaxial layer, which forms the major active volume of the sensor. The

\footnotetext{
${ }^{10}$ Note that the attenuation length of X-rays in silicon is in the order of the thickness of the epitaxial layer of CPS. Therefore, this assumption forms a rather rough approximation.
} 
"calibration peak" is associated with the depleted volume of the sensor. The related CCE is commonly accepted to amount $100 \%$. This is as signal electrons cannot escape from the strong electric fields found in this depletion zone.

The "calibration peak" is used to measure/calibrate the gain of the pixels and the readout chain of the CPS. In the example, the charge of 1640 electrons corresponds to an amplitude of 195 AD-units. The position of the "collection peak" is found at 65 ADU. Comparing it with the value for the calibration peak, one obtains the average $\mathrm{CCE}_{\text {Seed }}$ (roughly $30 \%$ ) of the epitaxial layer. Fig. 9b shows the spectrum as obtained from the summed amplitude of four pixels. Here, the "collection peak" is found at a position of about 155 ADU, which points to a $\mathrm{CCE}_{4}$ of about $80 \%$. By summing up the signal of all, typically less than $5 \times 5$ pixels of a cluster, one would typically obtain a $\mathrm{CCE}_{25}$ of $100 \%$ for good, non-irradiated sensors. Note that the calibration peak position found in the summed amplitude spectra is not reliable. For SB-pixels, the position of all peaks it biased by pixel charge clearing (see Sec. 3.2.2 and the reading is only reliable if this effect is suppressed e.g. by cooling.

The charge collection spectra may also be used to obtain an indicative measure of the depleted volume of the pixel. To do so, the number of entries of the calibration peak $\left(N_{1}\right)$ and the number of entries of the charge collection peak $\left(N_{25}\right)$ are extracted from the charge collection for the seed pixel and the one for 25 summed pixels respectively. The depleted volume $V_{d e p l}$ of the pixel is then given with:

$$
V_{\text {depl }} \approx \frac{N_{1}}{N_{25}} V_{\text {pixel }}
$$

The active volume $V_{\text {pixel }}$ of the pixel has to be extracted from the known pixel pitch and thickness of the epitaxial layer.

\subsubsection{The ${ }^{90} \mathrm{Sr}$ amplitude spectrum - signal charge and SNR}

The best laboratory measure for the signal charge and the SNR of the sensors is provided by illuminating the sensors with hard $\beta$-rays as for example emitted by a ${ }^{90} \mathrm{Sr}$. The $\beta$ rays interact approximatively like minimum ionizing particles and the related amplitude spectrum follows a Landau-distribution. As for the ${ }^{55} \mathrm{Fe}$ spectrum, the indicated charge changes if the signal of multiple pixels of a hit cluster are summed up. Note moreover that, depending on the authors, either the most probable value or the mean value of the asymmetric distribution is communicated.

A ${ }^{90} \mathrm{Sr}$ amplitude spectrum may be biased as $\beta$-rays injecting a charge at the lower side of the Landau distribution may not be detected at all by irradiated detectors. This scenario may be excluded by checking the number of entries of the plots. One may use the signal to noise ratio (SNR) of an ${ }^{90} \mathrm{Sr}$ spectrum, which is defined as the most probable charge according to the Landau distribution divided by the average high frequency noise (see above) as a first indicator for the capability of a sensor to detect minimum ionizing particles. As a good rule of thumb, an SNR $>15$ is required for a good particle detection. 


\subsubsection{Pixel clearing constant}

Pixels with SB-structure (see Sec. 3.2.2 rely on a controlled clearing of the pixel capacitance of the signal $U_{S}$, which is in the simplest case done by a biasing diode. This clearing follows in good approximation an exponential function:

$$
U_{S}(t)=U_{S}(t=0) \cdot \exp \left(-\frac{t}{\tau}\right)
$$

Measuring the clearing time is complicated by the fact that the effect is steered by an interplay between the charge collection diode and the entrance node of the on-pixel pre-amplifier. The relevant scale of signal charges is $\sim 100 e$. Both aspects exclude a test of the amplifiers with external instruments.

A solution consists in pulsing the pixel and observing the signal decay by means of the standard readout chain. In the frequent case that the pixels don't integrate a dedicated pulse generator, the pulsing may done by detecting photons from a ${ }^{55} \mathrm{Fe}$-source, which provide a signal amplitude corresponding to the one of the calibration peak. Hereafter, the indicated signal after CDS is recorded for multiple integration times. This signal shows negative sign as it corresponds to the charge injected by the clearing mechanism. Fitting the results over time provides a good estimate of $\tau$, provided that this constant is reasonably slower than the integration time. 


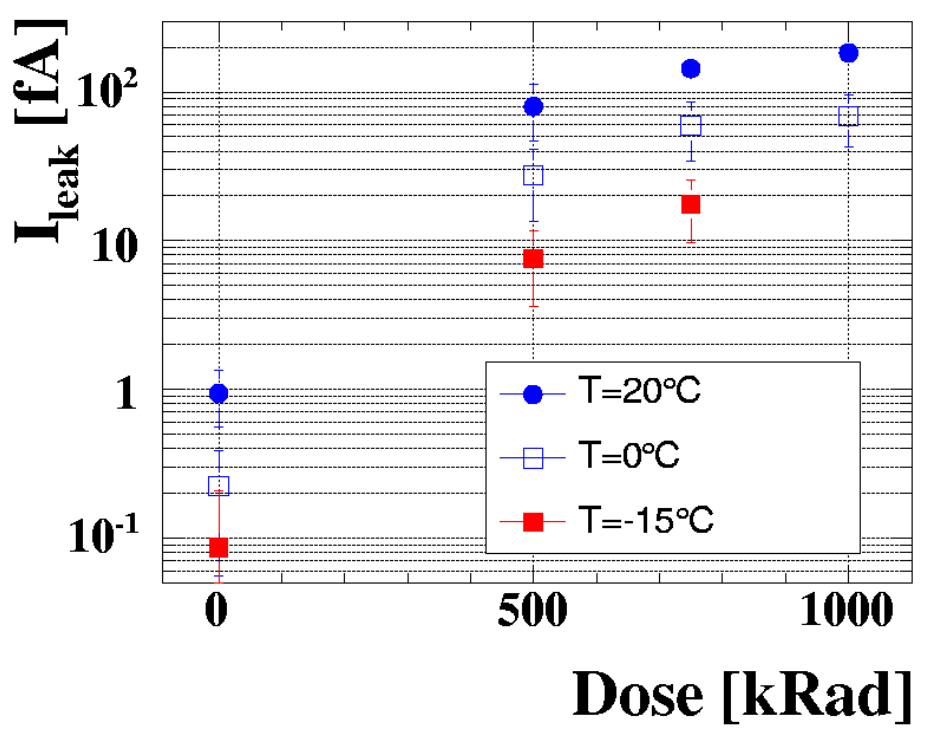

Figure 10: Leakage current of SUCCESSOR-1 a CPS imager with low resistivity epitaxial layer as function of total ionizing dose and temperature $\left(T_{\text {Coolant }}\right)$. From [54]. See original work for details on the device.

\section{$5 \quad$ Effects of integrated ionizing radiation}

\subsection{Increase of leakage currents and shot noise}

\subsubsection{T-pixel}

5.1.1.1 Leakage current The most prominent effect of ionizing radiation in CPS consists in an increase of the leakage currents of the pixel. This was first reported in a study with CPS used for optical imaging, which were exposed to $30 \mathrm{Mrad}$ [55]. The amount of the increase is in general specific to the individual CPS design and the production process. As illustrated in Fig. 10, the current may scale a fraction of a fA to few $\sim 100 \mathrm{fA}$ per diode after a dose of $1 \mathrm{Mrad}$ [54]. Despite this is not easy to see in the logarithmic plot, the leakage current increases, within an anticipated $10 \%$ dosimetry uncertainty, linearly with the total ionizing dose. A proportionally factor of $1.9 \mathrm{aA} / \mathrm{krad}, 6.6 \mathrm{aA} / \mathrm{krad}$ and $17.5 \mathrm{aA} / \mathrm{krad}$ may be extracted for a $T_{\text {Coolant }}$ of $-15{ }^{\circ} \mathrm{C}, 0{ }^{\circ} \mathrm{C}$ and $20{ }^{\circ} \mathrm{C}$ respectively. Note that those values are considered as device specific and provided without any claim of generality.

In 3T-pixels, the additional leakage current may discharge the pixel capacitance prior to the readout and thus saturate the pixel. This is not of worry if the integration time of the pixel is sufficiently fast to reset it in time. Alternatively, the current may be reduced by cooling. Note that also the pixel-to-pixel fluctuations of the current increases. This feature may challenge the dynamic of the amplification chain. Moreover, it makes it impossible to discriminate the signal of irradiated 3T-pixels with a common threshold.

A plausible source of the additional leakage current is generated by the surface defect states located at the interfaces between $\mathrm{Si}$ and thick $\mathrm{SiO}_{2}$ located nearby the diode. This is illustrated at the right side of Fig. 11a, which displays a simplified design of a related charge collection diode and indicates the location of the $\mathrm{N}$-well implantation and of the thick oxide in a qualitative way. One observes that the radiation induced surface defects found at the interface between the thick $\mathrm{SiO}_{2}$ and the $\mathrm{Si}$ generate thermal currents, which may be collected by the $\mathrm{n}$-well implantation. This would be further eased in case the positive charge build-up 

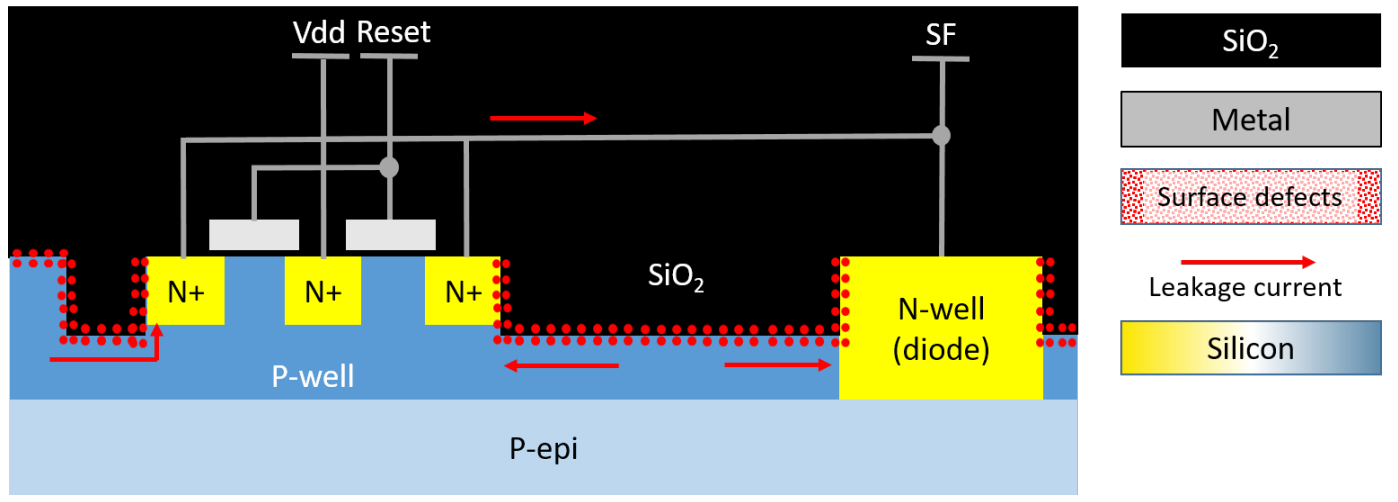

(a) Radiation soft pixel: A cross-section of an enclosed reset transistor (left, see also Fig. 1d) and of a charge collection diode (right) is shown. Vulnerable, thick $\mathrm{SiO}_{2}$ structures are placed nearby the diode. The radiation induced defects at the surface of those structures generate leakage currents, which are collected by the diode. Additional leakage current is collected by the outer ring of the reset transistor and forwarded to the pixel node.

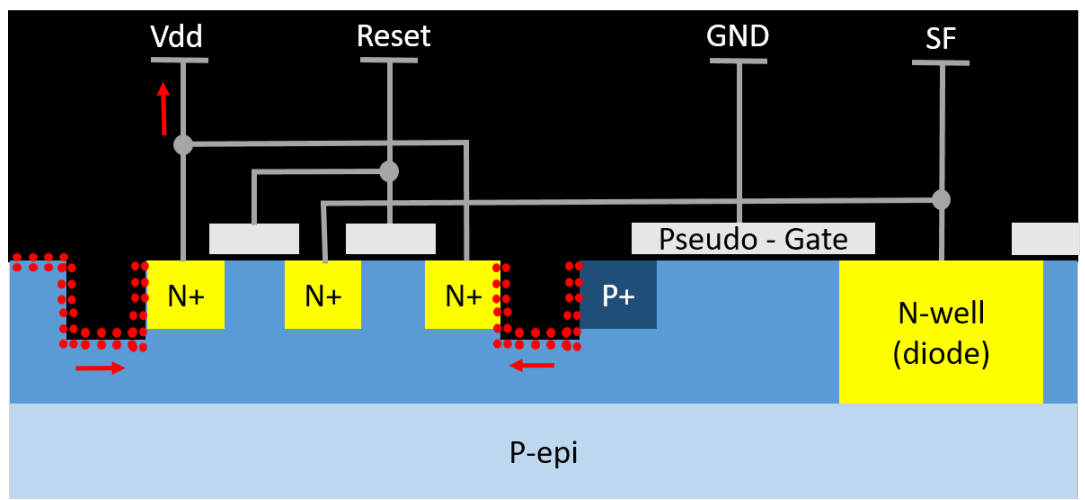

(b) Radiation hardened pixel: Here, the protected inner ring of the reset transistor is connected to the pixel node. Leakage currents collected by the outer ring are thus evacuated to the power supply. A p-diff $(\mathrm{P}+$, alternatively $\mathrm{n}$-diff may be used) guard ring is placed around the n-well diode. A "pseudo gate" guard ring forces the generation of thin and thus radiation hard $\mathrm{SiO}_{2}$ between this guard ring and the $\mathrm{N}$-well diode.

Figure 11: Cross-section of a radiation soft and a radiation hardened 3T-pixel with enclosed reset transistor. Surface damage is indicated by red dots, radiation induced leakage currents by red arrows, the black surfaces represent $\mathrm{SiO}_{2}$, the dark blue surfaces the p-well. 

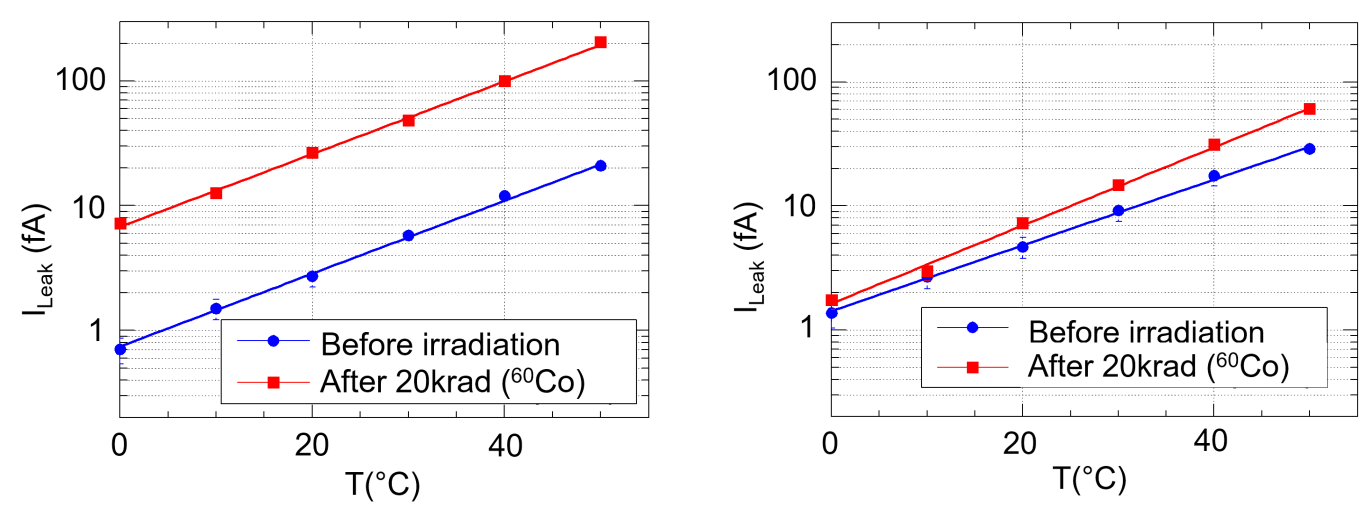

Figure 12: Leakage current on the radiation soft (left) and the radiation hard (right) 3T-pixel of MIMOSA-9 before and after an irradiation with $\sim 20 \mathrm{kRad} \gamma$ - rays from a ${ }^{60} \mathrm{Co}$ - source. One observes the increase of the leakage current to be substantially reduced in the radiation hard design. From [50].

related to the defects deforms the bands and creates thus a conduction channel along the surface toward the diode.

Design efforts to reduce the leakage current of the collection diodes aimed to remove thick $\mathrm{SiO}_{2}$ structures like the STI from the vicinity of the diode [56]. A successful design is illustrated in the right part of Fig. 11b. It consists in surrounding the n-well of the diode by a circular gate, which is terminated in a p-diff guard ring. By doing so, the thick silicon located nearby the diode is replaced by the thin, radiation hard gate oxide. The doping gradient generated with the p-diff guard ring generates a built in voltage, which repulses free electrons from the diode. Alternatively, a n-diff guard ring may be used, which absorbs the currents. In the most successful attempt to realize this structure, the gate voltage was set to the internal GND of the sensor. Applying other voltages by means of external voltage sources was tested but found to inject first of all an unacceptable pick-up noise. Note that the implementation of the above mentioned strategy with a standard CMOS process may require to bend design rules, which caused issues in some cases. A comparison between the measured leakage currents of a 3T-pixel with standard and radiation hardened diode is shown in Fig. 12. In this case, the guard ring was realized as n-diff. One observes that the radiation induced increase of the leakage current is reduced by factors.

Besides the diode, the reset transistor of a 3T-pixel collects (bulk-to-source) leakage currents. This is as the source of the reset transistor and the surrounding p-doped bulk form another pn-junction, which may be larger in size than the diode. For 3T-pixels using a "radiation hard" enclosed transistor, this transistor may from the dominating source of leakage currents. A cross-section of the non-optimized reset transistor is shown at the left side of Fig. 11a One observes that the outer ring of the transistor is placed once more in direct contact with thick $\mathrm{SiO}_{2}$ structures, which turns into a collection of leakage currents. In the non-optimized version of the transistor, this current enters the pixel node and adds to the 
diode leakage current. The easy solution to this issue consists in connecting the pixel node to the protected, inner ring of the transistor (see Fig. 11b. Indeed a pixel using a radiation protected diode and an optimized reset transistor layout was found to have a leakage current $31.6 \pm 0.2 \mathrm{fA}$ after $1 \mathrm{Mrad}$ and at $T_{\text {Coolant }}=10{ }^{\circ} \mathrm{C}$ while an otherwide identical pixel with non-optimized reset transistor with showed a leakage current of $286.9 \pm 1.7 \mathrm{fA}$ under the same conditions (see [50], P. 148). This suggests that the leakage current of a non-optimized transistor may dominate the one of a charge collection diode by far.

5.1.1.2 Noise The radiation induced increase of the leakage current $I_{l}$ may increase the shot noise of the pixel. This noise is caused by the fact that the current is transported by discrete charge carriers. The charge $Q_{L}$ collected during one integration time $t_{\text {int }}$ of the pixel is composed from $N$ individual electrons. Therefore:

$$
Q_{L}=N \cdot e=\int_{0}^{t_{\text {int }}} I_{l} d t
$$

According to Poisson statistics, the uncertainty of $\mathrm{N}$ amounts $\Delta N=\sqrt{N}$. The electron equivalent noise $Q_{L}$ created by this current can be considered as the fluctuation of the collected charge. It amounts:

$$
\Delta Q_{L}=e \cdot \sqrt{N}=\sqrt{e \cdot I_{l} \cdot t_{\text {int }}}
$$

The shot noise adds quadratically to the initially dominating noise $\Delta Q_{A} \approx 10$ e of the preamplifier:

$$
\Delta Q \approx \sqrt{Q_{A}^{2}+e \cdot I_{l} \cdot t_{\text {int }}}
$$

It is suited to push the total noise of the amplifier to an unacceptable value, which restricts the functional radiation tolerance of the devices. As $\Delta Q_{L}$ depends on the leakage current and on the integration time, the radiation hardness limit of a device may be extended with cooling and accelerating the readout. Despite both strategies are restricted by practical constraints, the radiation tolerance of a given sensor may vary by more than one order of magnitude depending on the operation conditions.

\subsubsection{SB-pixel}

5.1.2.1 Leakage current In first order, SB-pixels react rather friendly to radiation induced increases of the leakage current $I_{L}$. The current is compensated by the biasing diode, which cancels it from the output signal of the pixel after applying CDS and hampers the pixel from saturating. It is thus possible to discriminate the signal of multiple, irradiated SB-pixels with a common threshold. However, the variations in $I_{L}$ create a number of rather relevant, indirect effects, which should be considered.

The best way to approach the indirect leakage current effects of a SB-pixel is to consider it as a combination of the forward biased diode and a current source, which is realized by the reversed biased collection diode. Both components form a voltage divider ${ }^{11}$. The working point is set by the voltage drop $U_{B}$ caused by $I_{L}$ in the biasing diode. As the $I_{L}$ of cooled

\footnotetext{
${ }^{11}$ See schematics in Fig. 6 .
} 


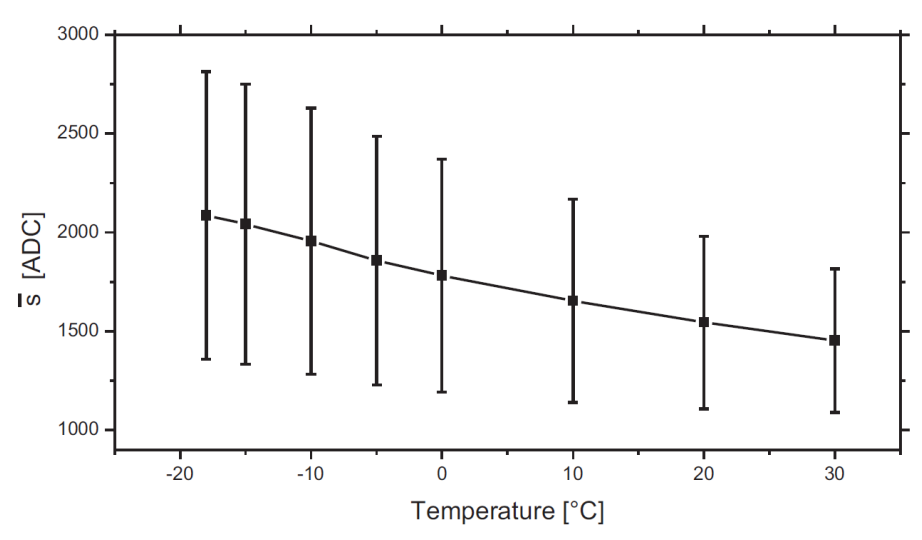

Figure 13: Mean value and RMS (error bars) of the dark signal distribution of SB-pixels on a non-irradiated CPS based on a $0.35 \mu \mathrm{m}$ process. $I_{L}$ increases with $T$. The shift of the working point and the reduction of the RMS is visible. Saturated pixels were removed. $1 \mathrm{ADC}$ unit $\approx 4 e$. From [57].

and non-irradiated diodes is measured in individual electrons per readout cycle, $U_{B}$ tends to be smaller than the $\sim 0.7 \mathrm{~V}$, which are usually considered as a good value for macroscopic currents passing a forward biased diode. This working point moves once $I_{L}$ increases due to increasing temperature or irradiation (see Fig. 13), which should be considered in the design of the amplification chain. Moreover, the working point is subject to important pixel-to-pixel fluctuations in $I_{L}$. In contrast to the situation in the 3T-pixel, this variation tends rather to decrease with increasing $I_{L}$.

The leakage current of SB-pixels is hard to measure. As similar diodes are used, their leakage currents should match the numbers known from 3T-pixels the related radiation hardening strategies apply. Quite obviously, no leakage current from a reset transistor is expected in SB-pixels.

5.1.2.2 Signal clearing The combination of the biasing diode and the pixel capacitance $C$ can be considered as a kind of RC-element, where the resistivity of the diode is steered by the leakage current. This element removes the signal charge $Q_{S}$ from the pixel capacitance. This removal process follows in good approximation [56]:

$$
Q_{S}(t)=Q_{S}(t=0) \cdot \exp \left(-\frac{t}{\tau}\right)
$$

In this equation, $t$ is the time after the signal generation and $\tau$ the time constant of the clearing process, which is given with:

$$
\tau \approx \frac{n \cdot k_{B} \cdot T \cdot C}{e} \cdot \frac{1}{I_{L}+I_{S}}
$$

Here, $I_{S}$ is the saturation current of the biasing diode, $I_{L}$ the leakage current of the collection diode, $1<n<2$ represents the emissivity of the biasing diode, $e$ the elementary charge, $k_{B}$ the Boltzmann constant and $T$ the temperature. For ideal diodes with the size and doping of a typical biasing diode, $I_{S}$ is roughly in the order of $1 \mathrm{fA}$, which can be neglected with respect to the leakage current of irradiated pixels.

The clearing process of the pixel can be measured with the procedure discussed in Sec. 4.3.6. A result of this measurement is shown in Fig. 14a, which shows the related curve for a SB-pixel before and after irradiation. As expected, the higher leakage current of the 


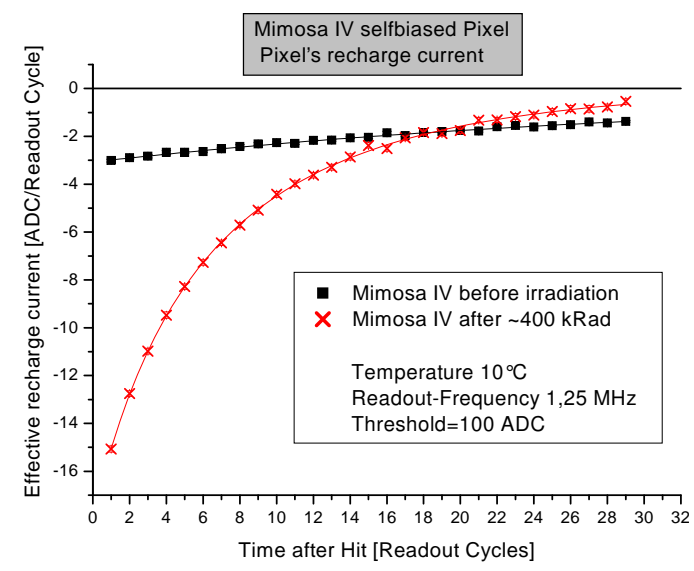

(a) Measured recharge current of a nonirradiated and irradiated SB-pixel as function of the radiation dose.

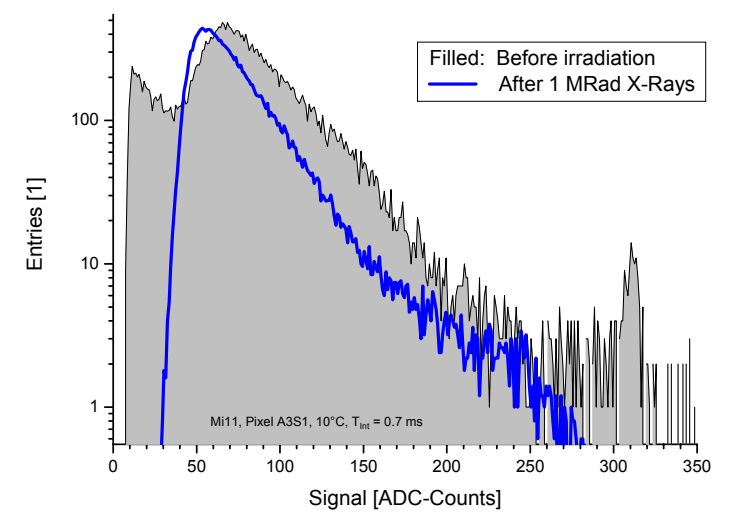

(c) Seed spectrum of a SB-pixel before and after irradiation with X-rays. A mild accelerated clearing smears the calibration peak and reduces the signal amplitudes.

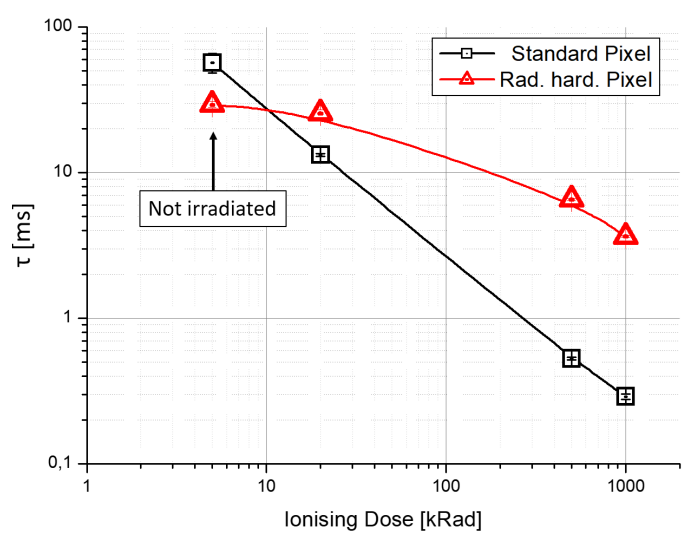

(b) Clearing time constant $\tau$ as a function of the radiation dose at $T=10^{\circ} \mathrm{C}$ for a standard SB-pixel on a LR epitaxial layer.

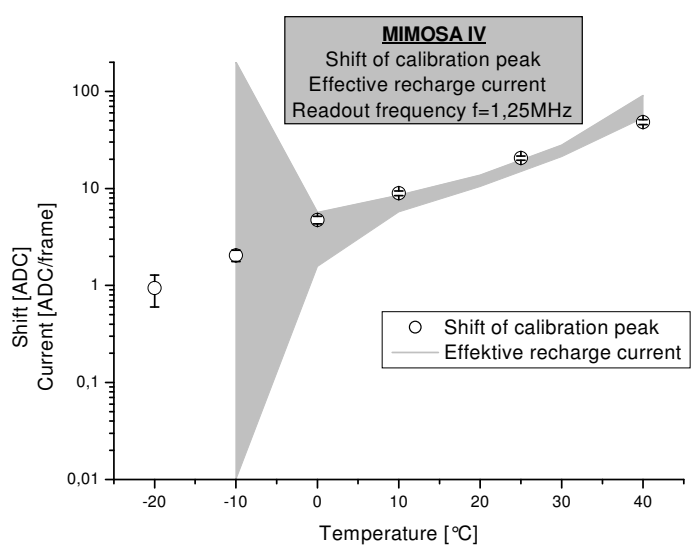

(d) Correlation between the displacement of the calibration peak and the amplitude of the recharge current. See text. From [50.

Figure 14: Illustration of the major effects caused by accelerated signal clearing. The plots are based on measurements taken with different sensors, more details are found in [50, 56]. See text. 
irradiated collection diode turns into an increase of the amplitude of the recharge current and a shortening of the clearing constant $\tau$. The evolution of the clearing time with increasing radiation dose is illustrated in Fig. 14b. One observes the clearing time $\tau$ of the non-irradiated sensor to amount several $10 \mathrm{~ms}$ before irradiation. The SB-pixel with radiation hardened diode shows a slight disadvantage with respect to the standard design prior to irradiation. After irradiation, the time constant is accelerated by orders of magnitude and the radiation hardened pixel shows better performance already after small doses. This is as the increase of leakage current is suppressed in this pixel.

Once $\tau$ approaches the integration time $t_{i n t}$ of the pixel, a part of the signal is cleared prior to the readout. This is illustrated in Fig. 14c, which shows the charge collection spectrum of an affected pixel. One observes a shifting of small calibration peak toward smaller values 12 . This reflects the clearing of a fraction of the signal between the time of the particle hit and the time of readout. As the time of particle impact is random, this time span avaliable for signal clearing follows a flat distribution, which ranges from zero to $t_{\text {int }}$. This creates the smearing of the peak.

The modification of the spectrum due to signal clearing is sometimes confused with a loss of signal amplitude due to reduced CCE. However, both are separate processes. This is illustrated in Fig. 14d, which shows an example of the correlation between the observed displacement of the calibration peak (points) and the effective recharge current generating the clearing as shown in Fig. 14b. Both quantities were recorded as a function of the temperature in order to modify the leakage current and thus $\tau$. The effective recharge current is displayed as a grey shaded area. The width of this area which reflects the uncertainties of the related measurement. One observes that the amplitude of the clearing process is reduced with decreasing temperature. This is as $\tau$ becomes longer while the integral of the exponential, which relates to the charge co be cleared, remains constant. The observed and expected clearing are in good accordance, which supports the above mentioned understanding. Doing this test allows to separate signal clearing from reduced CCE, mostly as effects reducing the CCE are typically not strongly temperature dependent. Note that besides cooling, reducing the integration time forms an obvious mean to reduce unwanted signal clearing.

5.1.2.3 Noise As for the 3T-pixel, the radiation induced increase of the leakage current $I_{L}$ generates shot noise in SB-pixels. Due to the continuous bias, the shot noise is not described with the model used for the 3T pixel (equation 15). However, one may still assume the leakage current $I_{L}$ removes a charge $Q_{\text {out }}=I_{L} \cdot t_{\text {int }}$ out of the pixel capacitance during the integration time $t_{i n t}$. At the same time, this charge is replaced by the biasing diode, which injects $Q_{i n}=I_{L} \cdot t_{\text {int }}$. Obviously, in thermal equilibrium, the total charge injected to the capacitor $Q_{L}$ is given with:

$$
Q_{L}=Q_{\text {in }}-Q_{\text {out }}=0
$$

While there is no net charge transfer, the noise $\Delta Q$ can be estimated by means of Gaussian error propagation with:

$$
\Delta Q_{L}=\sqrt{\left(\Delta Q_{\text {in }}\right)^{2}+\left(\Delta Q_{\text {out }}\right)^{2}}=\sqrt{2 \cdot \Delta Q_{\text {in } / \text { out }}}
$$

\footnotetext{
${ }^{12}$ See Sec. 4.3 .4 on how to read ${ }^{55} \mathrm{Fe}$ charge collection spectra.
} 


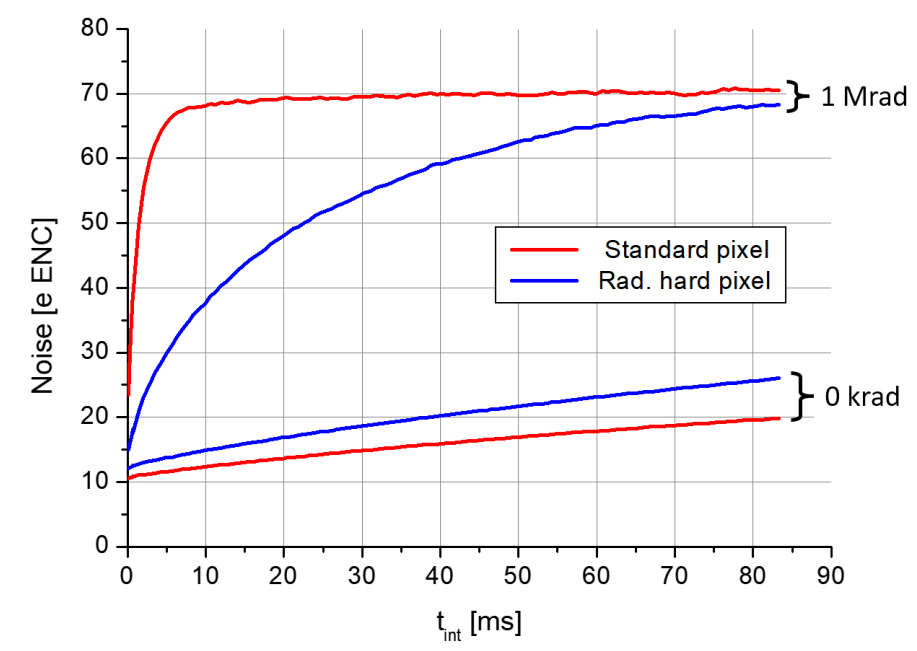

Figure 15: Noise of a standard and a radiation hardened SBpixel as function of the radiation dose and the integration time. The data was taken at $T=10^{\circ} \mathrm{C}$. After [56].

By using the relation between shot noise and leakage current from equation 14 , one obtains:

$$
\Delta Q_{L}=\sqrt{2 e \cdot I_{l} \cdot t_{\text {int }}}
$$

Consequently, one expects SB-pixels to show a by $\sqrt{2}$ higher shot noise then related 3T-pixels.

Unlike to 3T-pixels, the shot noise of SB-pixels does not increase toward infinity with increasing integration time. This is as the signal clearing of the pixel acts also for the small "signals" of the noise fluctuations. In a rough picture, one can therefore distinguish two regimes. In case $t_{\text {int }} \ll \tau$, equation 20 may be applied. In case $t_{\text {int }} \gg \tau$, the integration time of the pixel is effectively limited by the recharge process. In this case, equation 20 becomes:

$$
\Delta Q_{L}=\sqrt{2 e \cdot I_{l} \cdot \tau}
$$

This equation predicts a saturation of the noise for very high leakage currents (determining a very fast $\tau$ ) and long integration times. By using equation 17 and assuming $I_{S} \ll I_{L}$, one may estimate the saturation value:

$$
\Delta Q_{L}=\sqrt{2 n \cdot k_{B} \cdot T \cdot C}
$$

Unfortunately, the saturation of the pixel noise remains of modest practical interest as the saturation occurs by construction only if the clearing time becomes too short for effective charged particle detection.

As shown in Fig. 15, this saturation effect is indeed observed. The figure shows the noise of a SB-pixel with standard diode and one with hardened diode as a function of the radiation dose and the integration time. The related measured values of $\tau$ can be extracted from Fig. 14b As suggested by Equation 22, the noise runs into saturation for the radiation soft, irradiated pixel. Going into detail, one notes that the numerical value of the saturation level of $\sim 70 e$ ENC suggests a plausible pixel capacitance of $\sim 10 \mathrm{fF}$. The full saturation is only reached after $t_{i n t} \approx 10 \tau$. The same observation is made for the radiation hardened diode, which saturates 13 at the highest integration times shown thanks to its lower $I_{L}$ and longer $\tau$. The non-irradiated pixels do not reach saturation.

\footnotetext{
${ }^{13}$ The saturation of this pixel is more rigorously shown by additional measurements presented in [50], which also vary the temperature.
} 
As expected, the previously described approaches for reducing the leakage current of the diodes of 3T-pixels also reduce the noise of the SB-pixel. In the region of interest with less than few ms integration time, the difference is rather sizeable. This holds despite the fact that the radiation hardened pixels show a slightly higher noise before irradiation.

\subsubsection{Clamping pixel}

A number of studies were carried out in order to understand the tolerance of clamping pixels to ionizing radiation. A comparative study on the radiation tolerance of different sensing elements shows that a combination of the clamping structure with a 3T-sensing element turns into an unacceptable fixed pattern noise already after moderate radiation doses of $300 \mathrm{krad}$ [58]. This may be as the clamping structure is not suited to compensate the leakage currents and, more importantly, the pixel-to-pixel fluctuations of thus current. The increase of the fluctuations with increasing radiation dose turns into an increased fixed pattern noise. This feature was not observed for a pixel design relying on a SB-structure, which resisted reasonably well to doses of up to $300 \mathrm{krad}$.

Radiation studies beyond this dose revealed that the clamping pixel shows a shift of the threshold voltages and once more a increase of fixed pattern noise [52] in a pixel realized in a $0.35 \mu \mathrm{m}$ CMOS process. This effect could be reduced by means of thermal annealing. A potential origin of this effect is a radiation induced bulk to source leakage current of the clamping transistor, which discharges the clamping node. This effect was not observed in clamping pixels relying on a $0.18 \mu \mathrm{m}$ production process, which showed satisfactory performances up to $1.6 \mathrm{Mrad}$, which was the highest radiation dose applied [59].

\subsection{Effect of ionizing radiation to the charge collection}

As ionizing radiation does in first order not create bulk damage, which would reduce the lifetime of signal electrons, one does not expect this radiation to deteriorate the charge collection efficiency of a sensor. However, an impact on the charge collection efficiency was observed in a specific case [54] with a sensor manufactured in a $0.35 \mu \mathrm{m}$ CMOS process with an only $\sim 4 \mu \mathrm{m}$ thick epitaxial layer. The 3T-pixels had a pitch of $\sim 20 \mu \mathrm{m}$ and all transistors of the pixels were designed as enclosed transistors. Due to this feature and the use of an at least moderately deep sub-micron process, the sensor was hoped to be particularly radiation tolerant. Instead, a about 50\% drop of the CCE was observed after an irradiation of $400 \mathrm{krad}$. This effect was associated to a depletion of the p-well layer, which was intended to isolate the enclosed reset transistor from the sensing element. According to this interpretation, the depletion manifested itself once the fields of the $3.3 \mathrm{~V}$ potential of the outer ring of the transistor were complemented by the fields caused by radiation induced positive oxide charge. Due to this depletion, the transistor acted as parasitic collection diode. The issue was solved by inverting the layout of the transistor such that outer ring of the transistor was put on a lower potential. Other sensors with similar layout, which were produced in a different CMOS process, did not show this feature for doses of up to 1 Mrad [50]. The effect is therefore considered as specific to a, today obsolete, production process. However, it should be remembered as a warning, that ionizing may cause unexpected effects, which sometimes depend on 
undocumented features and modifications of the CMOS-processes used.

\subsection{Results from beam test campaigns}

A detailed study comparing the performances of SB-pixels with standard and radiation hardened design in beam was made with the sensor MIMOSTAR-2, which featured two matrices with each $128 \times 64$ pixels with $30 \times 30 \mu \mathrm{m}^{2}$ pitch [60]. The readout was done in serial analogue mode and the integration time was varied between 4.1 and $0.8 \mathrm{~ms}$ by changing the readout clock frequency. The test was carried out with a focus of using CPS in the STAR PiXeL detector, which was believed to require a dose of up to $50 \mathrm{krad}$ at this time. Operating the sensors after this dose was complicated by the long integration times and the need for a high, typically $+30{ }^{\circ} \mathrm{C}$ operation temperature, which created a relatively high leakage current and the related shot noise and signal clearing issues. It was observed that both, the standard and radiation hardened pixels reached a detection efficiency of $>99 \%$ if being operated at this unfavourable temperature and at a integration time of $4.1 \mathrm{~ms}$. Once being irradiated to $20 \mathrm{krad}$, the standard sensors showed only a poor detection efficiency of $<90 \%$. This performance drop was first of all created by an increase of the shot noise, which increased from initially $10 e \mathrm{ENC}$ to slightly below $35 e \mathrm{ENC}$. The pixels with radiation hardened diode remained operational up to the highest dose applied. As expected from the above discussions on the properties of the SB-pixel, best performances were reached at short integration times and low temperatures as both steps reduce the shot noise and the pixel clearing. A better than $99 \%$ detection efficiency was observed for the radiation hard pixel after applying a dose of $50 \mathrm{krad}$ at a temperature of $30{ }^{\circ} \mathrm{C}$ and a integration time of $2 \mathrm{~ms}$. For a temperature of $40{ }^{\circ} \mathrm{C}$, this efficiency dropped to about $96.6 \%$. Despite this was not analysed explicitly, ${ }^{55} \mathrm{Fe}$ spectra recorded for the same chip in [61] suggest that the primary failure mechanism of the standard pixel was a too fast signal clearing. This effect was substantially alleviated by radiation hard design.

Results of a beam test with an MIMOSA-26 (see Sec. 3.3 for technical data) with high resistivity epitaxial layer, which was irradiated with $150 \mathrm{krad}$ X-rays is reported in [62]. The sensor was operated intentionally under unfavourable conditions $\left(T=+35{ }^{\circ} \mathrm{C}\right.$ and an integration time of $230 \mu \mathrm{s}$ instead of the nominal $115 \mu \mathrm{s}$ ). This was done once more in the prospective of operating the sensor at the STAR PiXeL detector. The longer integration time was chosen to emulate the properties of the final sensor (MIMOSA-28/ULTIMATE), which was not yet available at the time. A detection efficiency for $120 \mathrm{GeV} / \mathrm{c}$ pions of $99.8 \%$ in combination with a dark rate of $10^{-5}$ was reported for the sensor flavour hosting a $15 \mu \mathrm{m}$ thick $400 \Omega \cdot \mathrm{cm}$ high resistivity wafer. This exceeds the performances of the non-irradiated MIMOSA-26 relying on a low resistivity epitaxial layer.

The FSBB-M0 prototype (see Sec. 3.3 for technical data), which integrated clamping pixels into the Tower $0.18 \mu \mathrm{m}$ process resisted to doses of up to $1.6 \mathrm{Mrad}$ in combination with a non-ionizing dose of $10^{13} \mathrm{n}_{\mathrm{eq}} / \mathrm{cm}^{2}$ with $99 \%$ detection efficiency and a dark hit rate of $10^{-5}$ according to a beam test with [59]. 


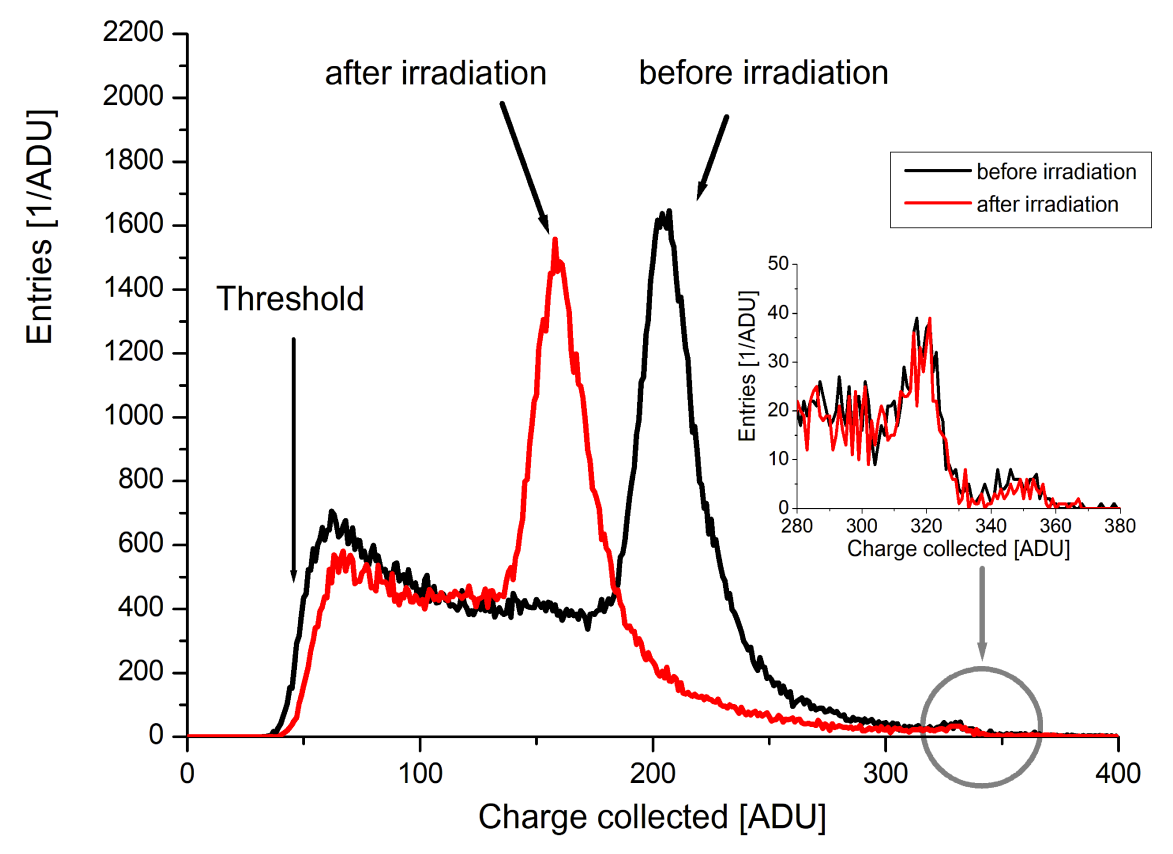

Figure 16: Illustrative picture of the impact of bulk damage on CPS with $10 \Omega \cdot \mathrm{cm}$ epitaxial layer. The ${ }^{55} \mathrm{Fe}$ amplitude spectrum (sum signal of four pixels) of a virgin and a sensor irradiated with $3 \times 10^{12} \mathrm{n}_{\text {eq }} / \mathrm{cm}^{2}$ neutrons is shown. The zoom shows the single pixel amplitude spectrum in the region of the calibration peak. From [64]. See text.

\section{Effects of integrated non-ionizing radiation}

\subsection{Sensors with $10 \Omega \cdot \mathrm{cm}$ epitaxial layer}

The first generation CPS relied on non-optimized CMOS processes, which provided a sensitive volume of about $10 \Omega \cdot \mathrm{cm}$ resistivity and a nominal bias voltage of 3 or $5 \mathrm{~V}$. Most sensors were equipped with 3T-pixels. The depletion depth of the related charge collection diodes is $\lesssim 1 \mu \mathrm{m}$ [63]. Most of the signal charge is collected slowly by thermal diffusion. In this early phase, a number of different combinations of pixel pitches and epitaxial layer thicknesses were explored. The typical values for the pixel pitch amounted $10-40 \mu \mathrm{m}$, the thickness of the epitaxial layer started from $4 \mu \mathrm{m}$.

\subsubsection{Charge collection efficiency, gain and noise}

First observations were made with non-optimized prototype sensors, which relied on 3T-pixels with $20 \mu \mathrm{m}$ pitch [64]. The sensors were irradiated with $\sim 1 \mathrm{MeV}$ reactor neutrons and their charge collection efficiency (CCE) was measured with a ${ }^{55} \mathrm{Fe}$-source . For practical reasons, this CCE was expressed for groups of four pixels, which should not be confused with the CCE of the seed pixel. An illustrative example of the amplitude spectra obtained is shown in Fig. 16. One observes that the charge collection peak 14 found in the spectrum is shifted along $\mathrm{x}$-axis, which is related to a reduced CCE in the non-depleted active volume in the medium.

\footnotetext{
${ }^{14}$ See Sec. 4.3 .4 for an introduction on how to read ${ }^{55} \mathrm{Fe}$ amplitude spectra.
} 


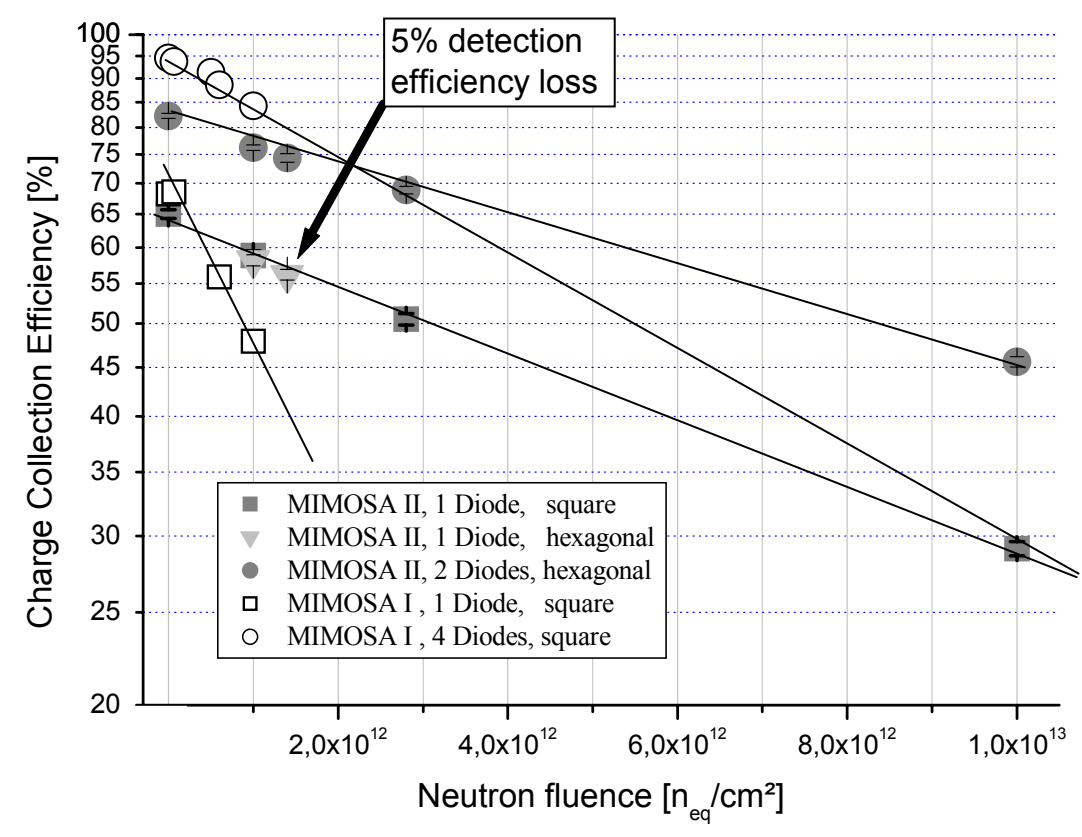

Figure 17: Charge collection efficiency for groups of 4 pixels as a function of the radiation dose. The data was taken with an ${ }^{55} \mathrm{Fe}$-source with MIMOSA-1 (14 $\mu \mathrm{m}$ epitaxial layer) and MIMOSA-2 (4 $\mu \mathrm{m}$ epitaxial layer). Both sensors had a pixel pitch of $20 \mu \mathrm{m}$. Some pixels hosted more than one diode, which were connected in parallel. Lines to guide the eye. From [50].

The seed pixel spectrum shown in the zoom does not indicate any displacement or significant modification in terms of entries. This shows that the gain of the CPS did not change as consequence to the irradiation. Moreover, it suggests that the properties of the depleted volume of the charge collection diode was not modified and thus excludes, in accordance with measurements on the doping concentrations performed much later, significant modifications in the doping concentration of the epi-layer.

The sensors showed a deterioration of the charge collection efficiency (CCE) after being irradiated with doses of $\sim 1 \mathrm{MeV}$ reactor neutrons beyond some $10^{11} \mathrm{n}_{\mathrm{eq}} / \mathrm{cm}^{2}$. As shown in Fig. 17, this $\mathrm{CCE}_{4}$ dropped to $30-50 \%$ after $10^{13} \mathrm{n}_{\mathrm{eq}} / \mathrm{cm}^{2}$. The functional radiation tolerance limit of this kind of devices was evaluated with a beam test and found to amount $\sim 10^{12} \mathrm{n}_{\mathrm{eq}} / \mathrm{cm}^{2}$ [50]. A S/N=15 (most probably value) was extracted as necessary requirement to obtain the $95 \%$ detection efficiency for minimum ionizing particles, which were considered as minimum for considering the sensor as operational.

From the findings, it was suspected that, due to the radiation induced reduction of the minority charge carrier life time, the signal charge carriers generated in the epitaxial layer might have recombined before being collected. Indeed, Eq. 3 suggests a charge carrier lifetime of $\sim 400 \mathrm{~ns}$ for a radiation dose of $10^{12} \mathrm{n}_{\mathrm{eq}} / \mathrm{cm}^{2}$. This value can be compared with a mea- 
surement on the charge collection time, which was carried out with a similar sensor relying on a $14 \mu \mathrm{m}$ thick epitaxial layer. The sensor was illuminated with pulses from a $1060 \mathrm{~nm}$ IR laser and the delay of the pixel signal was measured. This delay was interpreted as the charge collection time of the pixel, which is reported to amount $\sim 100 \mathrm{~ns}$ for pixels incorporating one diode and $\sim 60 \mathrm{~ns}$ in case the pixel hosts four diodes, which are connected in parallel [40, 65]. Accounting for this charge collection time, one expects to collect $86 \%$ or $77 \%$ of the initial charge after a dose of $10^{12} \mathrm{n}_{\mathrm{eq}} / \mathrm{cm}^{2}$ for a pixel with four and one diode per pixel respectively. This simple assumption reproduces the data shown on the related sensor (MIMOSA-1 in Fig. 17) with reasonably good precision.

A much higher radiation tolerance was reported for a sensor built in a $0.25 \mu \mathrm{m}$ CMOS process with an $8 \mu \mathrm{m}$ epitaxial layer [66]. It hosted different kinds of 3T-pixels with $15 \mu \mathrm{m}$ pitch and diodes of either $3 \times 3 \mu \mathrm{m}^{2}$ or $1.2 \times 1.2 \mu \mathrm{m}^{2}$ surface. One structure was equipped with enclosed transistors and one with four parallel diodes per pixel. Some samples were irradiated with $24 \mathrm{GeV} / \mathrm{c}$ protons at the CERN-SPS and intentionally not powered during irradiation. They were kept at $-20{ }^{\circ} \mathrm{C}$ hereafter for both, test and storage. The test demonstrated that the sensors remained operational up to the highest dose applied $\left(10^{15} \mathrm{n}_{\mathrm{eq}} / \mathrm{cm}^{2}\right)$, which demonstrated CPS to tolerate very high non-ionizing doses before breaking down. Surprisingly, no significant increase of the leakage currents is reported. Based on the $S / N$ as measured with a $\beta$-source, the authors concluded that the sensors still operate reasonably well after a dose of $10^{14} \mathrm{n}_{\mathrm{eq}} / \mathrm{cm}^{2}$.

This controversial conclusion is supported somewhat by the fact that one irradiated structure does indeed indicate a $\mathrm{S} / \mathrm{N}$ of 15 , which one can consider as a reasonable limit for efficient MIP detection. On the other hand, the measurements presented do not necessarily follow a clear trend. For example an insufficient $\mathrm{S} / \mathrm{N}$ of 7 is reported for the successful structure for the lower dose of $5 \times 10^{13} \mathrm{n}_{\mathrm{eq}} / \mathrm{cm}^{2}$. Moreover, the predicted charge carrier life time for a dose of $10^{14} \mathrm{n}_{\mathrm{eq}} / \mathrm{cm}^{2}$ amounts $\sim 4 \mathrm{~ns}$, which is dramatically shorter than the above reported charge collection time 15 .

It should be mentioned that measuring the small signal charge of CPS with $\beta$-rays alone creates room for a specific mistake. The charge deposit of those particles follows a broad Landau-fluctuation. Irradiated sensors may fail to detect $\beta$-rays contributing to the the lower region of this distribution, which moves the indicated mean value up. In addition, early experiments computed an individual discrimination threshold for each pixel, which smears the lower edge of the remaining Landau-distribution such the the remaining tail appears as a good distribution. The biased measurement indicates therefore an apparently perfect Landau distribution, which is however reduced in number of entries and indicates a too high $\mathrm{S} / \mathrm{N}$. It is well possible that the authors of the study would have arrived at different conclusions if they have had the possibility to cross-check their findings with a ${ }^{55} \mathrm{Fe}$-source or a particle beam. 


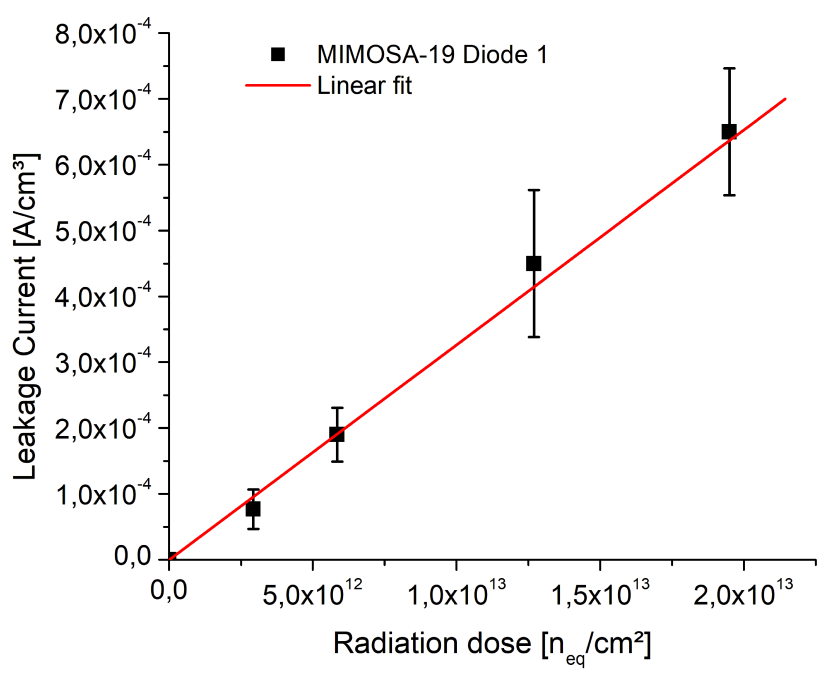

Figure 18: Measured increase of the radiation induced leakage current for a CPS with $12 \mu \mathrm{m} \times 12 \mu \mathrm{m}$ pixel pitch, a $39.6 \mu \mathrm{m}^{2}$ L-shaped diode and $\mathrm{a} \sim 11 \mu \mathrm{m}$ thick, low resistivity epitaxial layer. The uncertainty on the dosimetry amounts $\sim 10 \%$. The slope amounts $\alpha\left(20^{\circ} \mathrm{C}\right)=3.3 \times 10^{-17} \mathrm{~A} / \mathrm{cm}$ with an uncertainty of a small factor two (see text). Computed based on data from [67, 68].

\subsubsection{Leakage currents}

As introduced in Sec. 2.2, the radiation induced bulk leakage current $\Delta I$ of a PN-junction which was irradiated with a flux $\Phi$ of $\sim 1 \mathrm{MeV}$ reactor neutrons, is given with:

$$
\Delta I=\alpha(T) \cdot \Phi \cdot V
$$

A value of $\alpha\left(20^{\circ} \mathrm{C}\right)=4.0 \times 10^{-17} \mathrm{~A} / \mathrm{n}_{\mathrm{eq}} / \mathrm{cm}$ is given for $T=20^{\circ} \mathrm{C}$ and an annealing of $80 \mathrm{~min}$ at $60^{\circ} \mathrm{C}$. For depleted silicon detectors, $V$ denotes the depleted volume of the diode. For CPS relying on charge collection by diffusion, the equation states that electrons that electrons, which were thermally excited to the conduction band of the non-depleted epitaxial layer are not collected by the charge collection diodes. At the same time, this charge collection is obviously taking place for electrons, which were excited by a particle. It is a priori not obvious, why the charge collection process should be selective to the history of the electrons.

An experiment aiming to confirm the validity of Eq. 23 was carried out with the CPS prototype MIMOSA-19, which hosts 3T-pixels with $12 \mu \mathrm{m} \times 12 \mu \mathrm{m}$ pixel pitch, a $39.6 \mu^{2}$ L-shaped diode and a $\sim 11 \mu \mathrm{m}$ thick, low resistivity epitaxial layer. The leakage currents of this sensor were reported in [67] and the data was combined with recent measurements [68] on the depleted volume, which were performed according to the procedure discussed in Sec. 4.3.4 No particular care for a controlled annealing $\left(80 \mathrm{~min}\right.$ at $\left.60{ }^{\circ} \mathrm{C}\right)$ of the sensors was undertaken as the study aimed initially toward a different objective.

The result of the study is displayed in Fig. 18, which was plotted under the assumption that $V$ is identified with the depleted volume of the photo-diode. One finds the expected linearity of the leakage currents and $\alpha\left(20^{\circ} \mathrm{C}\right)=3.3 \times 10^{-17} \mathrm{~A} / \mathrm{cm}$, which confirms Eq. 23 . The difference with respect to the literature value may be caused by the uncertainties of the measurement of the depleted volume. More likely, it reflects that the sensors under test were subject to a (too) long room temperature annealing of radiation damages prior to the experiment.

\footnotetext{
${ }^{15}$ The $15 \mu \mathrm{m}$-pixels discussed here would plausibly show a moderately faster charge collection time than the $20 \mu \mathrm{m}$-pixels used for measuring the charge collection time. However, this is unlike to change the picture.
} 


\subsubsection{Radiation hardening strategies for CPS with low resistivity active medium}

Radiation hardening strategies for CPS with low resistivity active medium aim to alleviate the impact of the losses in signal amplitude. The strategies presented were developed for a use with simple CMOS processes, which do not allow for depleting the pixel, which reflects the technology available in the early stage of the R\&D on CPS. Instead of depleting, it was tried to accelerate the charge collection by shortening the diffusion paths of the signal electrons. This was done by reducing the thickness of the epitaxial layer, the pixel pitch and/or using multiple diodes per pixel. Moreover, it was tried if extending the epitaxial layer may increase the initial number of charge carriers generated to an extend, which overcompensates the reduced charge collection efficiency.

6.1.3.1 Thickness of the active medium The latter approach was driven to its extreme by implementing a CPS on a CMOS wafer without epitaxial layer. Instead, the pixels with $20 \mu \mathrm{m}$ pitch used the few $100 \mu \mathrm{m}$ thick and moderately $\left(\sim 10^{14} \mathrm{p} / \mathrm{cm}^{3}\right)$ doped substrate as active medium. The sensor showed a rather good initial $\mathrm{S} / \mathrm{N}$ and detection efficiency, which was considered as a proof that CPS do not necessarily require an epitaxial layer [69]. However, the width of the hit clusters was increased and a reduced spatial resolution for MIPs was observed and the radiation induced drop of the signal charge was found exceed the one known from the standard sensors. According to beam tests, the MIP detection efficiency of sensors being previously exposed to a neutron dose of $10^{12} \mathrm{n}_{\mathrm{eq}} / \mathrm{cm}^{2}$ dropped to only $\sim 90 \%$. Therefore, the modified sensors showed a lower radiation tolerance than the standard sensors with epitaxial layer (see [50], P. 160).

This weakness is plausibly related to the interface between the epitaxial layer and the substrate. In sensors with epitaxial layer, this interface creates a built in voltage, which hampers signal electrons from leaving the epitaxial layer and from getting lost in the deep substrate. Such, it increases the CCE of the sensor. In the absence of the interface, the CCE of the upper part of the active medium is reduced but the effective thickness of the active medium is increased. Consequently, one observes initially a higher signal charge. After irradiation, the life time of the electrons is reduced and electrons created in the deep substrate do not reach the charge collection diodes. Therefore, the disadvantage of the missing interface persists while the advantage of the larger effective thickness of the active medium vanishes.

The limitations toward the edge of thinner epitaxial layers is discussed in a study [70], which compared the performances of sensors build on epitaxial layers with a thickness of $4 \mu \mathrm{m}$, $11 \mu \mathrm{m}$ and $14 \mu \mathrm{m}$. The study estimates the charge signal and the $\mathrm{S} / \mathrm{N}$ of sensors (based on the known thickness of the epitaxial layer and the CCE as measured with ${ }^{55} \mathrm{Fe}$ ) as a function of the radiation dose. In accordance with Fig. 17, it is found that for pixels with $20 \mu \mathrm{m}$ pitch, the $\mathrm{CCE}$ of a $14 \mu \mathrm{m}$ thick epitaxial layer deteriorates faster than the one of a $4 \mu \mathrm{m}$ thick epitaxial layer. However, a higher signal is created by MIPs in the thicker epitaxial layer. This over-compensates the losses in CCE for all doses below the functional radiation limit of both devices. Therefore, the thicker epitaxial is found to be more radiation tolerant.

6.1.3.2 Pixels with multiple, parallel collection diodes The use of multiple diodes has repeatedly been considered as a mean to improve the charge collection performances 


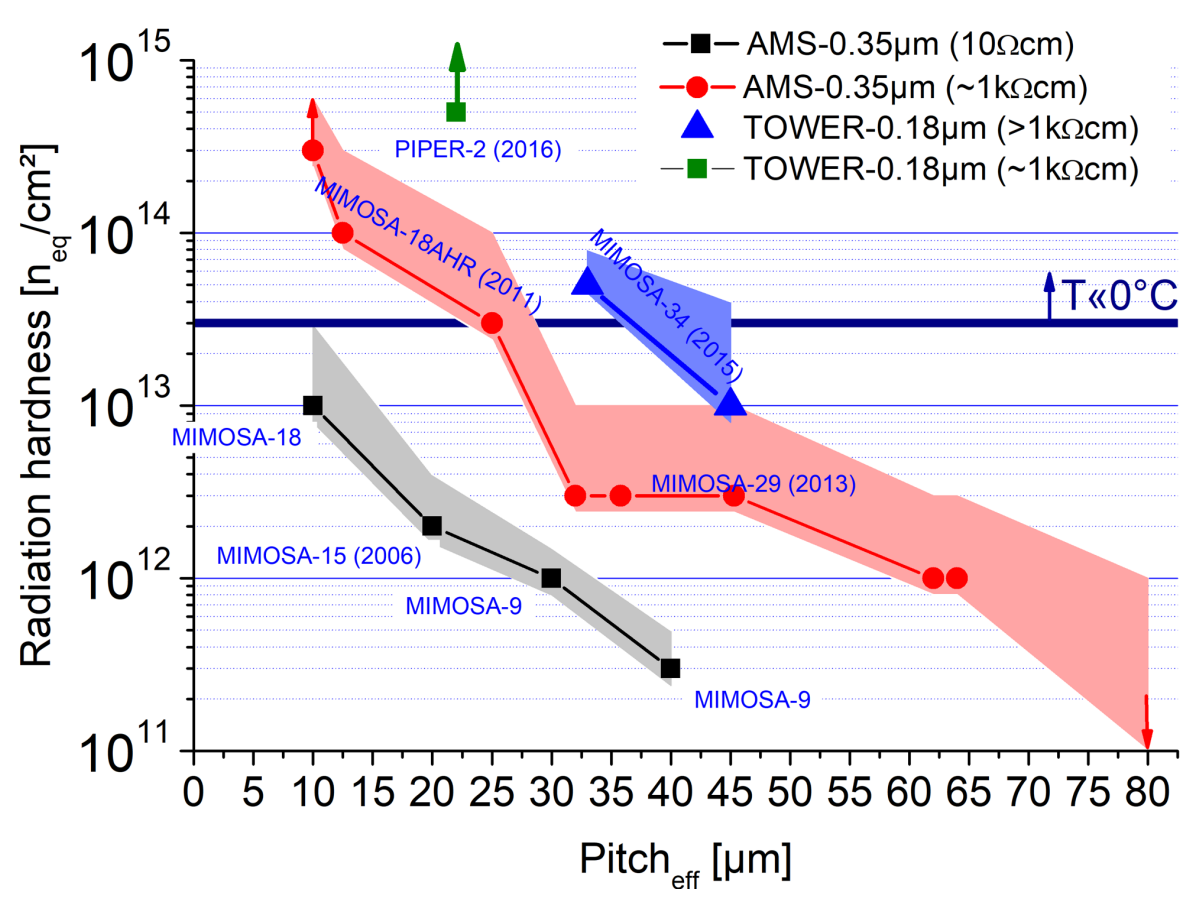

Figure 19: Compilation of the tolerance of CPS of the MIMOSA-series as a function of the effective pixel pitch and the technology. Compiled from [52, 71, 70, 72, 75, 73].

of CPS. Therefore, the technology was implemented into various early sensor prototypes [2, 40, 66]. The obvious draw back of the approach consists in the additional leakage current and increased pixel capacitance, which can be considered to scale approximatively with the number of diodes connected in parallel. The additional capacitance decreases the charge-tovoltage conversion gain of the pre-amplifier of the pixels and increases in consequence the noise of the device. E.g. in [2, a noise of $15 e$ ENC is reported for a pixel with $20 \mu \mathrm{m}$ pitch and one diode with a surface of $3.1 \times 3.1 \mu \mathrm{m}^{2}$. This compares to a noise of $31 \mathrm{e}$ ENC for the related version of this pixel hosting four diodes. In [70, it is estimated, if the advantage of the better charge collection efficiency dominates the disadvantage caused by the higher noise. It is concluded that the pixels hosting multiple diodes show a higher signal and that their CCE shrinks slower than the one of the pixels with individual diode. However, due to the higher noise, this advantage does not turn into a relevant advantage in terms of $\mathrm{S} / \mathrm{N}$ and radiation tolerance. Similar conclusions were drawn for pixels hosting diodes with a very high surface 67.

6.1.3.3 Pixel pitch Decreasing the pixel pitch reduces the diffusion paths between the impact point of the particles and the collection diodes. Moreover, the relation between the surface of the charge collection diode and the pixel surface is improved. One expects both modifications to accelerate the charge collection process and thus the CCE of irradiated pixels. As the capacitance of the charge collection diodes remains unchanged, no increase of the pixel noise is to be expected. Indeed, a strong correlation between the pixel pitch and the radiation 
tolerance of the sensors was observed (see Fig. 19). This was first discussed in [70] and [72] for SB-pixels relying on the AMS $0.35 \mu \mathrm{m}$ OPTO process and a $11 \mu \mathrm{m}$ thick epitaxial layer with a high doping $(\sim 10 \Omega \cdot \mathrm{cm})$ and confirmed with beam tests [50. As shown in the figure, the radiation tolerance of pixels with $40 \mu \mathrm{m}$ was found limited to $3 \times 10^{11} \mathrm{n}_{\mathrm{eq}} / \mathrm{cm}^{2}$ while sensors with $10 \mu \mathrm{m}$ pixel pitch were found to reach a radiation tolerance above $10^{13} \mathrm{n}_{\text {eq }} / \mathrm{cm}^{2}$. By fitting the data (and with all caution given the uncertainties), one may state that the radiation tolerance $\Phi_{\max }$ of the sensors as a function of the pixel pitch $10 \mu \mathrm{m} \leq \mathrm{p} \leq 40 \mu \mathrm{m}$ is given with:

$$
\Phi_{\text {max }} \approx 10^{15} \mathrm{n}_{\mathrm{eq}} / \mathrm{cm}^{2} \cdot\left(\frac{\mathrm{p}}{1 \mu \mathrm{m}}\right)^{-2.3}
$$

According to the equation, one may reach an impressive radiation tolerance by shrinking the pixels to a small pitch of few $\mu \mathrm{m}$, which is technologically possible in case a simple preamplifier and a sequential analogue pixel readout is employed. However, this approach turns into a large number of pixels per unit surface and thus the number per readout line (see Sec. 3.1.4. Consequently, sensors relying on pixels with a $p$ of few $\mu \mathrm{m}$ would show a rather slow time resolution, which is not acceptable for most applications.

6.1.3.4 Doping gradients The Fermi energy of doped semiconductors depends slightly on the doping concentration of this semiconductor. Consequently, doping gradients may be used to modify the bands of silicon and to generate built in voltages also outside PN-junctions. To understand this, one remembers that the location of the Fermi energy $E_{F}$ with respect to the intrinsic energy $E_{i}$ of p-doped silicon is given (see [39], P. 27) with:

$$
d E=E_{i}-E_{F}=k_{B} T \log \left(\frac{N_{A}}{n_{i}}\right)
$$

In semiconductors, the distance between $E_{i}$ and the potential of the valence and the conduction band depends by definition not on the doping concentration. Moreover, in thermal equilibrium and in the absence of depletion fields, the absolute potential of $E_{F}$ is constant over the full semiconductor. A change in $N_{A}$ creates therefore a shift for the absolute potential of $E_{i}$ and consequently for the potential of the bands. The potential difference between silicon doped with the concentrations $N_{A_{1}}$ and $N_{A_{2}}$ is given with:

$$
V=\frac{d E\left(N_{A_{1}}\right)-d E\left(N_{A_{2}}\right)}{e}=\frac{k_{B} T}{e} \log \left(\frac{N_{A_{1}}}{N_{A_{2}}}\right)
$$

The related electric field $\mathcal{E}$ amounts:

$$
\mathcal{E}=\frac{d V}{d x}=\frac{k_{B} T}{d x \cdot e} \log \left(\frac{N_{A_{1}}}{N_{A_{2}}}\right)
$$

Here, $d x$ denotes the distance between the points with the related doping concentrations. The drift speed of a signal electron exposed to a doping gradient is then given with:

$$
v=\mu \cdot \mathcal{E}=\frac{\mu}{d x} \frac{k_{B} T}{e} \log \left(\frac{N_{A_{1}}}{N_{A_{2}}}\right)
$$


At room temperature, the electron mobility $\mu$ is slightly above $1000 \mathrm{~cm}^{2} / \mathrm{V} / \mathrm{s}$ and the built in voltage caused by doping gradient of one order of magnitude amounts about $60 \mathrm{meV}$.

Assume $\mathrm{a} \sim 14 \mu \mathrm{m}$ thick epitaxial layer with ideal exponential doping gradient. Be the doping close to the diode for example of seven decades (e.g. from $10^{12} / \mathrm{cm}^{3}$ to $10^{19} / \mathrm{cm}^{3}$ ) between the most lowly doped point of the epitaxial layer (near the diode) and the highly doped substrate, this would turn into a sizable average field of about $30 \mathrm{kV} / \mathrm{m}$. This drift field is sufficient to move the electrons to a speed of $\sim 3 \mu \mathrm{m} / \mathrm{ns}$. This drift complements the diffusion and allows for crossing the epitaxial layer within less than $\sim 5 \mathrm{~ns}$, which may speed up the charge collection by one order of magnitude with respect to pure diffusion. One may therefore consider, that fields introduced even by moderately big doping gradients may be rather relevant.

An explicite attempt to profit from this effect was made at IPHC with the sensor MIMOSA21 ([58], P. 133). Two flavours of this sensor were manufactured in an $0.25 \mu \mathrm{m}$ BiCMOS process provided by an anonymous vendor. The sensor relied on a $9 \mu \mathrm{m}$ thick epitaxial layer with doping gradient. The precise doping profile and concentration was not documented. The process provided deep p- and n-well implantations and a part of the pixels was covered with deep p-well. Various pixel designs with a pixel pitch of $10 \mu \mathrm{m}$ and $20 \mu \mathrm{m}$ were tested. Among the parameters, which were modified in a systematic way, were the diode surface and the fraction of deep p-well in the pixel cell. In particular, it was tried to build a pixel, which is surrounded by a deep p-well ring. This design had the conceptual potential to concentrate the signal charge in one pixel diode and such to increase the $\mathrm{S} / \mathrm{N}$ of the seed pixel.

The results of the study were somewhat disappointing. Most of the different pixel designs showed an insufficient charge collection efficiency. This is interpreted as a consequence of a layer with increased p-doping concentration between the epitaxial layer and the collection diode. Due to the above mentioned built in voltages generated, this layer has the potential to deflect the signal charge away from the diode. Moreover, most pixels showed a sizeable leakage current, which was by significant factors above the ones reported for conventional pixels. A leakage current of several pA per pixel is reported for sensors, which were exposed to $500 \mathrm{kRad}$ and operated at room temperature. This high number exceeds the one observed in standard CMOS processes by at least one order of magnitude and reflects plausibly that the CMOS process was not optimized for building CPS.

However, an improved CCE was reported for a specific pixel with $2 \times 2 \mu \mathrm{m}^{2}$ pixel diode. In this pixel, most of the pixel surface was covered with ordinary p-well and deep p-well was arranged to a ring surrounding the pixel. This design had the conceptual potential to concentrate the signal charge in one pixel diode and such to increase the $\mathrm{S} / \mathrm{N}$ of the seed pixel, which complements a potential effect of the doping gradients. Indeed, an increase of the CCE from about $22 \%$ (seed pixel, reference pixel manufactured in $0.35 \mu \mathrm{m}$ AMS Opto) to $34 \%$ was reported. This CCE dropped to about 20 after a dose of $6 \times 10^{12} \mathrm{n}_{\mathrm{eq}} / \mathrm{cm}^{2}$, which appears by its own a rather good value. Unfortunately, the pixel showed a rather high noise of $19 e \mathrm{ENC}$ already prior to the irradiation. This, and the relatively thin epitaxial layer reduced the $\mathrm{S} / \mathrm{N}$ of the pixel to an unacceptable value ( 13 before irradiation, 7 after $6 \times 10^{12} \mathrm{n}_{\text {eq }} / \mathrm{cm}^{2}$ ). As by far better results were obtained with sensors relying on high resistivity epitaxial layers, the concept was abandoned. 


\subsection{Sensors with high resistivity $\sim 1 \mathrm{k} \Omega \cdot \mathrm{cm}$ epitaxial layer}

The tolerance of standard CPS to non-ionizing radiation is dominantly limited by the slow charge collection time of the sensor. This process may be accelerated by extending the depleted volume of the charge collection diodes, which increases the geometrical "capture cross-section" of the diodes for diffusing charge carriers. As discussed in Sec. 3.1.1, this extension may be reached by reducing the doping of the epitaxial layer. Doing so requires to process wafers featuring lowly doped epitaxial layers with existing CMOS processes. Since this option became available, it forms the standard technology for building CPS. To the best of the author's knowledge, all CPS used in big experiments of particle and heavy ion physics until nowadays, namely the STAR-PXL, the NA61-SAVD and the upcoming upgrade of the ALICE-ITS, rely on this technology.

\subsubsection{Charge Collection Efficiency}

The impact of using high resistivity (HR-) epitaxial layers as active medium of a CPS was first studied with TCAD [74] simulations. A pixel, which bases on a $0.6 \mu \mathrm{m}$ CMOS-process with a $15 \mu \mathrm{m}$ thick epitaxial layer was simulated assuming a standard low resistivity (LR, $\sim 10 \Omega \cdot \mathrm{cm})$ and a $\mathrm{HR}(1 \mathrm{k} \Omega \cdot \mathrm{cm})$ epitaxial layer [63]. Reducing the doping concentration is found to increase the thickness of the depleted layer of the collection diodes from $\sim 1 \mu \mathrm{m}$ to about $7 \mu \mathrm{m}$ at the available depletion voltage of $5 \mathrm{~V}$. The lateral radius of the depleted zone is increased from essentially the diode radius of $\sim 3 \mu \mathrm{m}$ (LR) to $\sim 7 \mu \mathrm{m}$ (HR). The depleted volume of the diode is thus dramatically increased. However, it remains insufficient to deplete the simulated $20 \mu \mathrm{m} \times 20 \mu \mathrm{m}$ pixels fully. The simulated sensor (MIMOSA-25) was realized. It exhibited an exceptionally high $\mathrm{S} / \mathrm{N}$ for MIPs of about 50. After being irradiated to a dose of $3 \times 10^{13} \mathrm{n}_{\mathrm{eq}} / \mathrm{cm}^{2}$, the sensors showed a remaining $\mathrm{S} / \mathrm{N}$ between 30 and 35 , which is a dramatic improvement as compared to LR-sensors and sufficient for a good MIP detection efficiency [63].

A more quantitative comparison between LR- and HR-sensors became possible once the AMS $0.35 \mu \mathrm{m}$ - OPTO process could be combined with both, a $10 \Omega \cdot \mathrm{cm}$ and a $400 \Omega \cdot \mathrm{cm}$ resistivity epitaxial layer. This allowed to realize sensors with both process flavours and to compare the performance of the otherwise identical devices [52, 71]. According to those studies, the charge collection efficiency of HR-sensors does not really exceed the one of good LR sensors. This is as the latter approaches $100 \%$ already. However, the charge is concentrated into the decisive seed pixel of the cluster. This is illustrated in Fig. 20a, which shows the average accumulated charge of pixel clusters generated by X-rays from a ${ }^{55} \mathrm{Fe}$-source. The $\mathrm{X}$-axis of the plot represents the number of pixels in the cluster considered for the charge measurement and the $\mathrm{Y}$-axis the related charge. One observes that the seed pixel $(N=1)$ collects a charge of 600 electrons in the LR-pixel and of slightly above 1000 electrons in the HR-pixel. The full signal charge is distributed to about 20 pixels for the LR-sensor is concentrated to roughly 4 pixels in the HR-sensor. The importance of this re-shaping of the clusters consists in the fact that the discrimination threshold of CPS, which is used for indicating the impact of one particle, acts mostly on the seed pixel.

Going into detail, one finds that the impact of using HR-epitaxial layers depends on the 


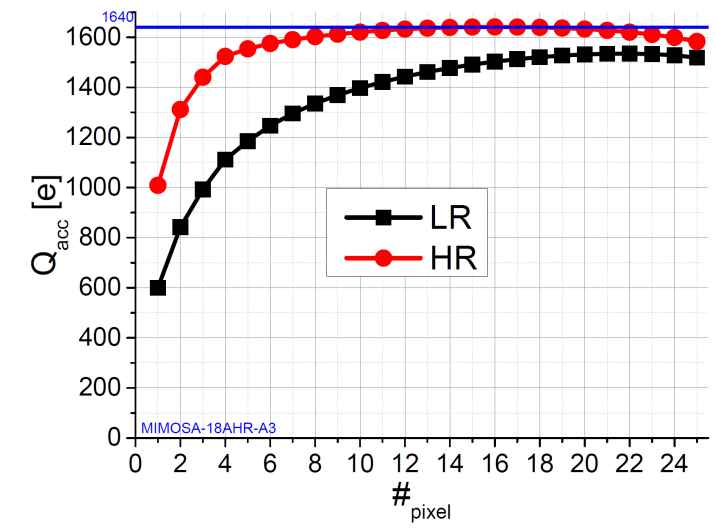

(a) Average summed signal charge from groups of $N$ pixels. MIMOSA-18, $10 \mu \mathrm{m} \times$ $10 \mu \mathrm{m}$ pixels. From [52].

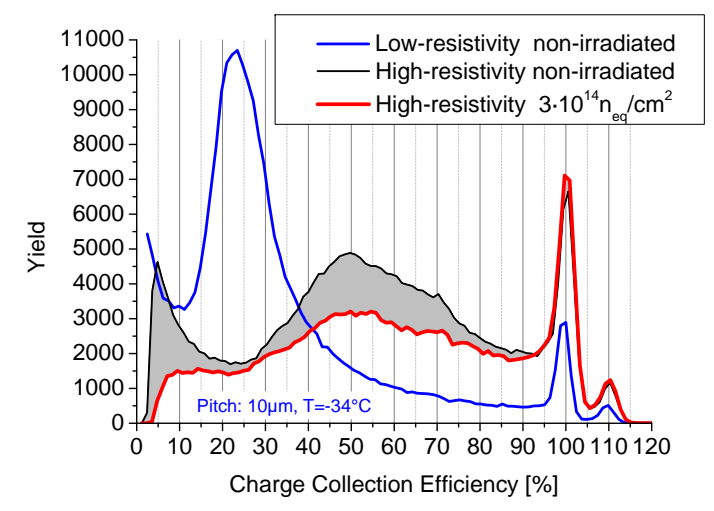

(c) Seed pixel spectrum of MIMOSA-18 with $10 \times 10 \mu \mathrm{m}^{2}$ pixels. From [71].

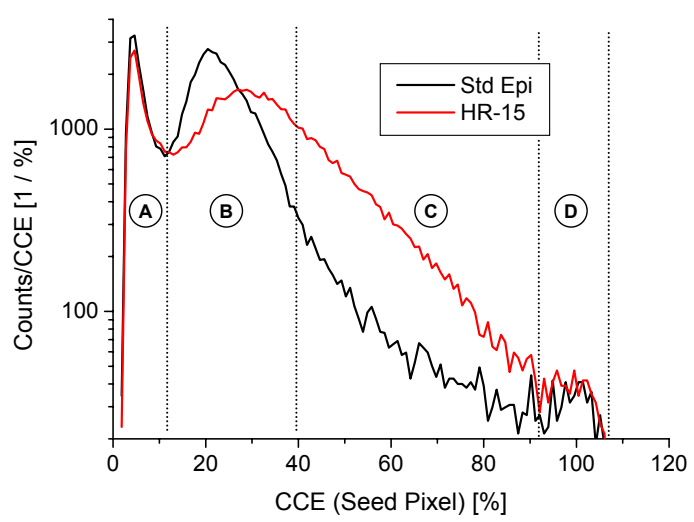

(b) Amplitude spectra of the clamping pixel of MIMOSA-26 $\left(18.4 \mu \mathrm{m}\right.$ pitch, $\left.V_{\text {depl. }}<1 \mathrm{~V}\right)$. From [43.

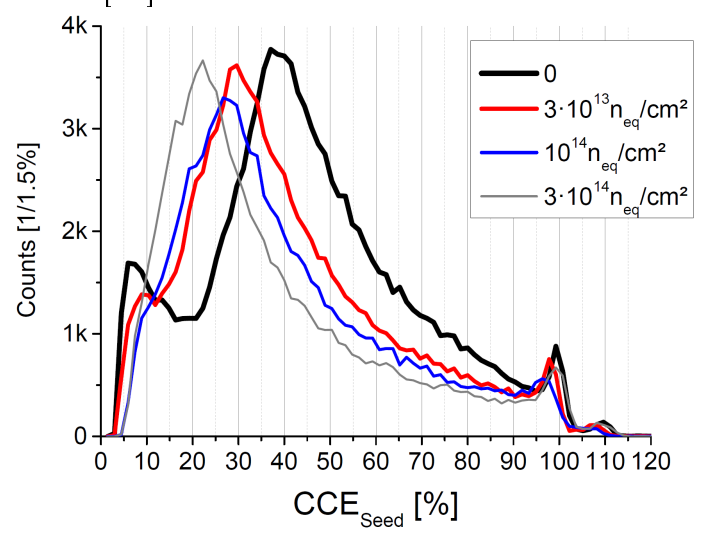

(d) Seed pixel spectrum of MIMOSA-18 with $25 \times 25 \mu \mathrm{m}^{2}$ pixels. From [52].

Figure 20: Comparison amplitude specta recorded with an ${ }^{55} \mathrm{Fe}$-source for pixels with low (Std.) and high resistivity (HR) epitaxial layer. 
sensor design. A first comparison, was made with the popular MIMOSA-26 [46, 52, 43 ] prototype $\left(18.4 \times 18.4 \mu^{2}\right.$ pixel pitch, clamping pixels with an effective depletion voltage $V_{\text {depl }} \lesssim 1 \mathrm{~V}$ ) and the related ${ }^{55} \mathrm{Fe}$ spectra are displayed in Fig. $20 \mathrm{~b}$. One observes that the number of entries in the calibration peak (region $\mathrm{D}$ in the figure) does not change significantly. However, the average charge collection efficiency is increased for the HR-sensor, which is in particular illustrated by the increased number of entries in region $\mathrm{C}$. Therefore one observes an improved $\mathrm{CCE}$ as expected from enlarging the depleted volume of the charge collection diodes but one does not observe this enlargement.

Additional studies were made with the MIMOSA-18AHR [52, 72] prototype $\left(10 \times 10 \mu \mathrm{m}^{2}\right.$ and $25 \times 25 \mathrm{\mu m}^{2}$, SB-pixels with $\left.V_{d e p l} \approx 3 \mathrm{~V}\right)$. The related spectra as recorded with the $10 \mu \mathrm{m}$ pixel of this chip are shown in Fig. 20c. One observes that the number of entries in the "calibration peak" of the spectrum of the HR-pixel exceeds the one of the LR-pixel by factors, which reflects the expected enlargement of the depleted volume. Again, the shift of the big peak of the spectrum indicates the expected increase of the CCE of the non-depleted active volume of the sensor. One may thus conclude from the observations that the use of a HR-epitaxial layer may or may not enlarge the depleted volume of the charge collection diode but the CCE from the non-depleted epitaxial layer is improved anyway.

A speculative explanation of those contradictory finding assumes that the p-doping concentration of the epitaxial layer increases nearby the wells, potentially due to a out-diffusion of p-dopands from the p-wells ${ }^{16}$. As this layer is created during the processing of the p-wells, its doping does not depend on the initial doping of the epitaxial layer. The n-well implantations of the diodes are embedded into this layer. Due to the small depletion voltage, the depleted volume of the charge collection diode of MIMOSA-26 remained within this layer. Therefore, the size of the depleted volume of the diode remains independent of the doping of the remaining epitaxial layer. The depletion voltage of MIMOSA-18AHR is sufficient to deplete the layer, to reach the lowly doped HR epitaxial layer and to generate a sizeable depleted volume there. The size of this depleted volume depends thus on the doping of the epitaxial layer. This does also improve the CCE from the epitaxial layer.

The reason for the improvement the CCE from the non-depleted volume of MIMOSA-26 remains speculative in this scenario. It might well be that the mentioned p-layer forms a shallow potential barrier, which hampers the signal electrons from entering the charge collection diode of the sensor and that this barrier is reduced/eliminated in the HR-sensor. Alternatively, one may speculate that the HR-sensor shows a beneficial doping gradient between epitaxial layer and substrate in analogy to what is discussed in Sec. 6.1.3.4. Testing both hypothesis would require a detailed knowledge on the doping profile of the epitaxial layer, which is unfortunately not available.

As illustrated in Fig. 20d for the MIMOSA-18AHR pixel with $25 \mu \mathrm{m}$ pitch, the CCE of the HR-sensor decreases with increasing non-ionizing radiation. On a qualitative level, this is equivalent to the observations from LR-pixels but higher doses are required for reducing the CCE. The latter suggests that the ambitioned acceleration of the charge collection time

\footnotetext{
${ }^{16}$ The existence of such a kind of layer was suggested by the observation that a minimum diode size is required in the AMS $0.35 \mu \mathrm{m}$ technology to connect the n-well and the epitaxial layer to a working charge collection diode, see [50] for details.
} 
was reached. It is also possible to build working pixels with a pitch above $40 \mu \mathrm{m}$. Studies on the radiation tolerance of such pixels are found in [52, 75] and cover pixels with a pitch of up to $80 \times 80 \mu \mathrm{m}^{2}$ as required for an accelerated rolling shutter readout. It is observed that the radiation tolerance of the pixels decreases in first order as a function of the surface of the pixels. A staggering of the pixels, which consists in shifting an elongated pixel by a half pitch, seems not to have significant impact on the radiation tolerance. The use of two or four diodes per pixel was also studied and the results confirm the mechanisms discussed in Sec. 6.1.3.2. However, a slight preference toward two diodes per pixel is observed for strongly elongated pixels. In pixels with four diodes, a region with good signal to noise and low charge sharing is indicated by a double peak in the amplitude spectrum. This region is likely located between the connected diodes at the center of the individual pixel. A region with worse performance is likely located in the periphery of the pixels and gives room for the creation of blind spots of poor CCE after moderate irradiation.

As shown in Fig. 20c the specific HR-version of the $10 \mu \mathrm{m}$ pixel of MIMOSA-18AHR showed an unusual response to a neutron dose of $3 \times 10^{14} \mathrm{n}_{\mathrm{eq}} / \mathrm{cm}^{2}$. Instead of the expected drop in the average CCE of the sensor, one observes a reduced number of entries in the charge collection peak. This suggests that the size of the active volume was reduced while the charge collection capabilities of the remaining volume remained unchanged. A more detailed analysis carried out with X-rays and $\beta$-rays [72] suggests that the effective thickness of the active medium was reduced by about $20 \%$ while most likely no blind spots are formed in the pixel.

An overview on the maximum radiation tolerance of pixels realized in HR-processes as a function of the pitch is once more given in Fig. 19. Most of the underlying test were made in the laboratory. A significant cooling of the sensors exposed to doses above $10^{13} \mathrm{n}_{\mathrm{eq}} / \mathrm{cm}^{2}$ was applied to control the leakage currents. A sensor was considered to resist to a given radiation dose if i) the $\mathrm{S} / \mathrm{N}(\mathrm{MPW})>15$ was observed for $\beta$-rays from a ${ }^{90} \mathrm{Sr}$-source, ii) the number of counts in the related amplitude spectra was not significantly reduced, iii) both, the ${ }^{90} \mathrm{Sr}$ and the ${ }^{55} \mathrm{Fe}$ amplitude spectra did not show hints for local inefficiencies. For the points of MIMOSA-29, the effective pixel pitch is given. This effective pitch is defined as $p_{\text {eff }}=\sqrt{A}$, where $A$ is the surface of the pixel. One finds that increasing the resistivity of the epitaxial layer from $10 \Omega \cdot \mathrm{cm}$ to $400-1000 \Omega \cdot \mathrm{cm}$ (AMS $0.35 \mu \mathrm{m}$ ) increases the tolerance of the pixels by roughly one order of magnitude. Again with all caution, one may fit the data of the sensors produced in AMS $0.35 \mu \mathrm{m}$ technology and state that those sensors show a reasonable radiation tolerance up to a dose of :

$$
\Phi_{\text {max }} \approx 4.5 \times 10^{17} \mathrm{n}_{\mathrm{eq}} / \mathrm{cm}^{2} \cdot\left(\frac{\mathrm{p}_{\mathrm{eff}}}{1 \mu \mathrm{m}}\right)^{-3.2}
$$

Increasing the resistivity substantially above $1000 \Omega \cdot \mathrm{cm}$ was studied with sensors based on the Tower/Jazz $0.18 \mu \mathrm{m}$. The use of this resistivity does not change the qualitative properties of the sensors but yields moderate additional factor in terms of radiation tolerance. 


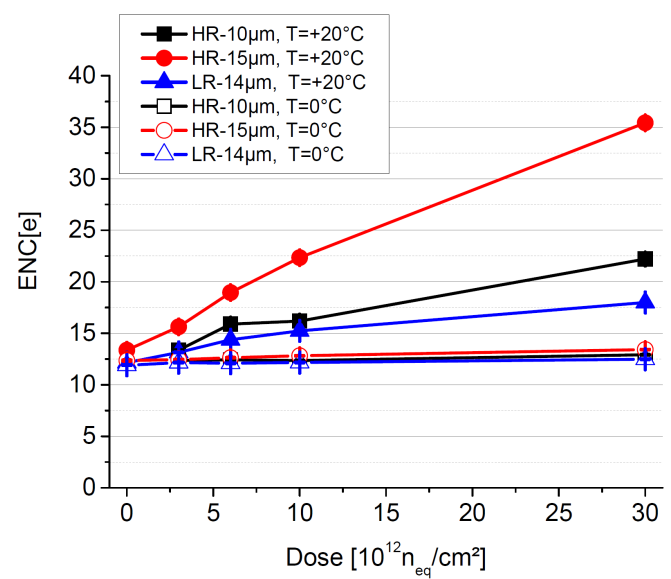

(a) Comparison of the noise of MIMOSA-26 sensors with HR- and LR-pixels as a function of the temperature and the radiation dose. The standard high resistivity sensor MIMOSA-26AHR relies on the $15 \mu \mathrm{m}$ thick HR epitaxial layer. From [52.

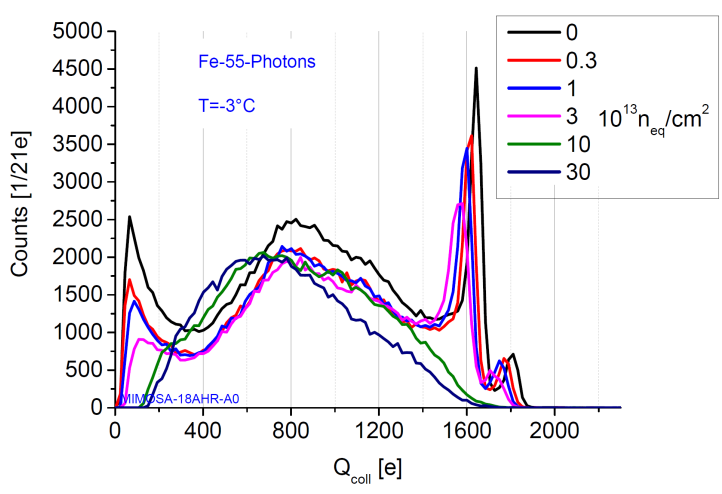

(b) Amplitude spectrum of a $10 \mu \mathrm{m}$ pixel of MIMOSA-18 as a function of the radiation dose. The increasing impact of the pixel clearing with increasing dose is visible [52].

Figure 21: Indirect consequences of the leakage current in CPS with HR-epitaxial layer.

\subsubsection{Noise and leakage current}

Only few systematic studies were performed in order to compare the leakage currents and the noise of irradiated LR- and HR-sensors of the MIMOSA-series. This is on one hand as the sensors of this generation were not designed to allow for a quantitative access to those currents (SB-pixels without direct external access to the biasing lines of the diodes). On the other hand, the leakage current increases observed at the doses of interest $\left(\sim 10^{13} \mathrm{n}_{\text {eq }} / \mathrm{cm}^{2}\right)$ were not considered as crucial as they remained on a level, which could be managed by means of moderate cooling and moderate fastly readout.

One study [52] comparing the leakage current and noise properties of LR- and HR-pixels relied on the (shot-) noise as an indirect measure for the current. A result of the study is shown in Fig. 21a, which displays the noise of MIMOSA-26 prototypes as a function of the epitaxial layer, the radiation dose and the temperature. Three epitaxial layers (low resistivity with $14 \mu \mathrm{m}$ thickness, high resistivity with $10 \mu \mathrm{m}$ and $15 \mu \mathrm{m}$ respectively) were considered. At a coolant temperature of $0{ }^{\circ} \mathrm{C}$, all options show the same noise. This suggests that the shot noise of the pixel diode is negligible at this temperature. For a temperature of $20^{\circ} \mathrm{C}$, the noise of the pixel relying on a $15 \mu \mathrm{m}$ thick HR epitaxial layer (HR-15) exhibits the highest noise, while the noise of the HR-10 pixel and of the pixel with low resistivity silicon remains substantially lower. This noise increase is likely caused by shot noise and points to a related increase of the leakage currents.

The increase of the leakage currents observed in the HR-10 and HR-15 sensors with respect to the reference sensor remains somewhat puzzling. On one hand, it is expected as one expects the HR-sensors to provide a more sizeable depleted volume and thus a higher radiation induced leakage current (see Sec. 6.1.2. On the other hand, the related increase of the depleted volume 
was not observed in the related ${ }^{55} \mathrm{Fe}$ amplitude spectra (see previous section). One might once more consider that the finding is driven by build-in-fields caused by doping gradients. As the doping profile of the different epitaxial layer was however not disclosed, this remains speculative and the sole conclusion of the study consists in the fact that the increase of the leakage currents of moderately irradiated sensors can be comfortably controlled by moderate cooling.

The limits of this strategy is illustrated by the amplitude spectrum of MIMOSA-18AHR pixels with $10 \mu \mathrm{m}$ pitch, which is shown in Fig. 21. One observes that the spectrum of the sensor operating at $t_{\text {int }}=6.6 \mathrm{~ms}$ and a temperature of $T=-3{ }^{\circ} \mathrm{C}$ is getting deformed with increasing radiation dose. This deformation becomes unacceptable above a dose of $3 \times 10^{13} \mathrm{n}_{\mathrm{eq}} / \mathrm{cm}^{2}$. It was associated with the clearing of the pixel and could be recovered by cooling the sensor to $T=-34{ }^{\circ} \mathrm{C}$ up to the highest dose (see Fig. 20c).

Given that the charge collection of HR-pixels was significantly improved as compared to LR-pixels, the radiation induced leakage current and the related pixel clearing becomes the dominating issue limiting the tolerance of HR-CPS to radiation. The limit of the radiation tolerance is determined by the temperature dependent leakage current and the integration time of the sensor. As a good rule of thumb, one may state that room temperature operation might be possible up to $\sim 10^{13} \mathrm{n}_{\mathrm{eq}} / \mathrm{cm}^{2}$. Above this limit, the use of a moderate cooling should be considered a loss in charge collection might set in for sizeable pixels. Starting from a dose of $10^{14} \mathrm{n}_{\mathrm{eq}} / \mathrm{cm}^{2}$, either a massive cooling or a very fast sampling of the pixel signal is required to handle the leakage current and only small pixels may show a sufficient charge collection.

\subsection{Results from beam tests}

Beam test with CPS being exposed to non-ionizing radiation were routinely undertaken and only a small subsection of the total results were published. Results shown in [50] confirm the above discussed dependency of the limits of the radiation tolerance of sensors with low resistivity epitaxial layer and the pixel pitch and demonstrate the radiation hardness limits of non-depleted CPS relying on high resistivity substrates.

A comparative study of the performances of MIMOSA-26 sensors (see Sec. 3.3) being implemented on a wafer with low and high resistivity epitaxial layers are shown in [43] and the related beam test results are displayed in Fig. 22. One observes that both sensor flavours create about the same spatial resolution of $\sim 4 \mu \mathrm{m}$ (a slighly better of $\sim 3.5 \mu \mathrm{m}$ is typically reached with a refined analysis). The "HR-15" sensors relying on the high resistivity epitaxial layer exhibit a substantially better combination of high detection efficiency and low dark rate than their low resistivity counter part. The sensor irradiated to $10^{13} \mathrm{n}_{\mathrm{eq}} / \mathrm{cm}^{2}$, which was the highest dose applied in this study, shows superior detection capabilities than the nonirradiated standard sensor. It is worth mentioning that the spatial resolution of the sensor is mostly not affected by the radiation damage, which may be considered as a general rule.

In [76], beam test performances of a chip with SB- and clamping pixels of $20 \times 20 \mu \mathrm{m}^{2}$ and $20 \times 40 \mu \mathrm{m}^{2}$ are reported. The sensor named MIMOSA-32 relied on the Tower/Jazz 180 nm CMOS process and was shown to resist once more to the highest radiation doses applied 

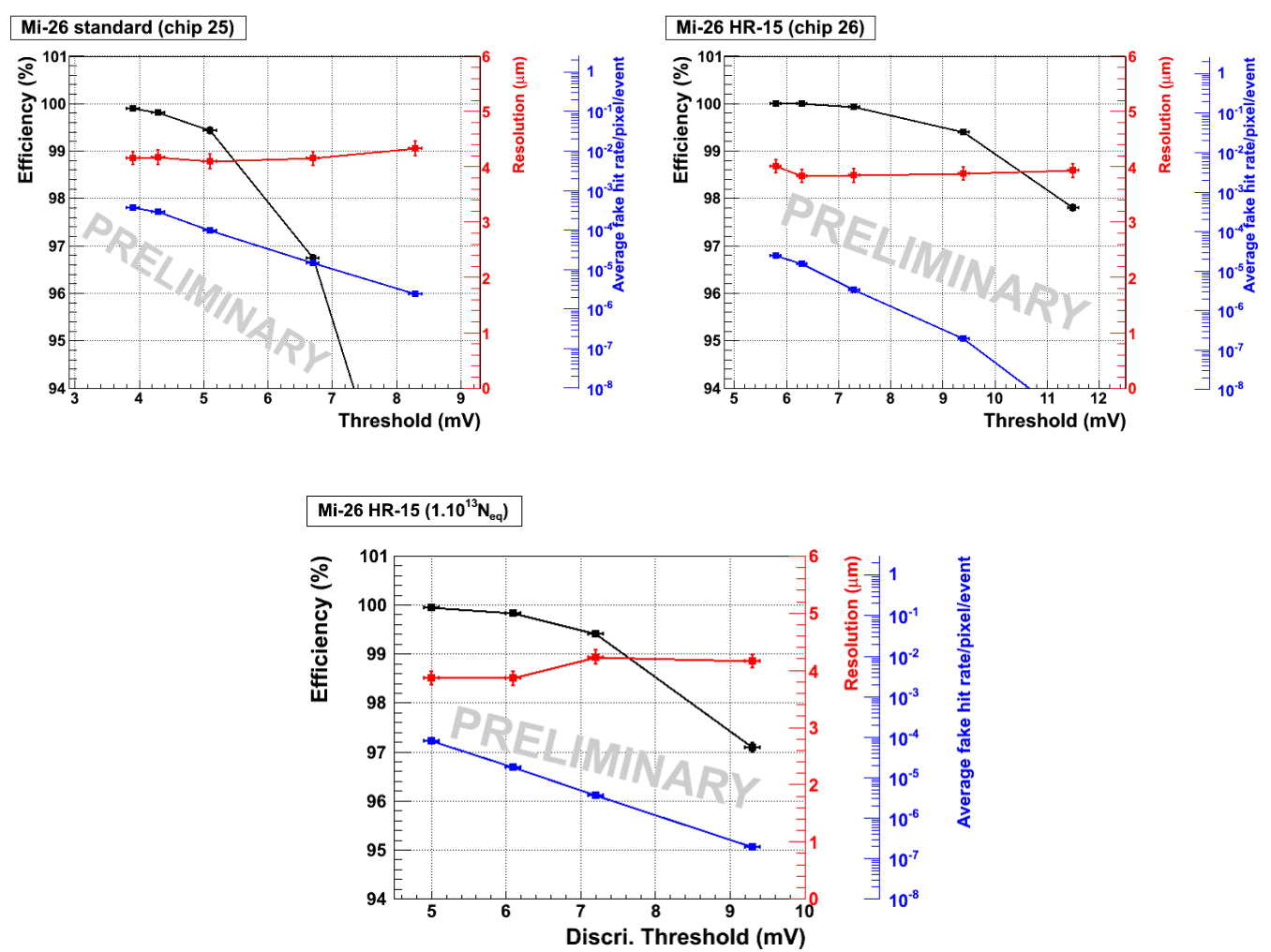

Figure 22: Beam test results for MIMOSA-26 with standard epi (upper left panel), HR-15 epi (upper right panel) and HR-15 epi irradiated with $10^{13} \mathrm{n}_{\mathrm{eq}} / \mathrm{cm}^{2}$ (lower panel). From [43].

( 1 Mrad plus $10^{13} \mathrm{n}_{\mathrm{eq}} / \mathrm{cm}^{2}$ at $T=+30^{\circ} \mathrm{C}$ ). Its tolerance to effects of high leakage current effects was improved by the relatively fast integration time of $t_{i n t}=32 \mu \mathrm{s}$. The sensor showed a good performance of $>99.5 \%$ after irradiation with exception of the $20 \times 40 \mu^{2}$ size pixel, which showed a $98 \%$ efficiency at $T=+30{ }^{\circ} \mathrm{C}$ but recovered to $99.5 \%$ once being cooled to $T=+15{ }^{\circ} \mathrm{C}$. Once more, the spatial resolution of the detector did mostly not change with radiation. A relatively high dark rate is reported, which was caused by RTS in the entrance stage of the in-pixel amplifier (see Sec. 8.1.3). A sensor relying on clamping pixels (FSBB-M0) resisted as well to a radiation dose of $10^{13} \mathrm{n}_{\mathrm{eq}} / \mathrm{cm}^{2}$, which was the highest dose applied [59]. 


\subsection{Outlook: Depleted MAPS}

\subsubsection{Depleted CPS, fundamental considerations and technological approach}

Depleting CPS comes with the need to apply high voltages to a substrate to a substrate with comparably high resistivity. While this was mostly impossible in the early days of CPS, the availability of modern CMOS processes with high resistivity substrate gave room for substantial progresses in the field. This work restricts itself on the results obtained with MIMOSA-series sensors. It should be mentioned that a large number of valuable activities were successfully carried out by other groups.

The particular challenge of depleting the active medium of CPS consists in the fact that the epitaxial layer of the sensors is thin as compared to the pixel pitch. Therefore, depleting a sensor is rather an issue of lateral depletion than of depleting into the depth. Moreover, applying a classical back bias in a straight forward way is hampered by the fact that the p-subst/p-epi/p-well structure of the standard CPS sensing element is conductive unless a full depletion is reached.

One option to overcome this issue is to replacing the initially small charge collection diode by a sizeable diode, which may contain a major part of the electronics. This creates a mostly planar junction, which is known to reach a depletion depth scaling with $\sqrt{U_{d e p l}}$. However, the big diodes come with sizeable capacities, which complicate the design of low noise sensors. Alternatively, it is tried to apply a high voltage to the conventional, tiny diodes. This allows for reaching very low diode capacities but the depletion the pixel, namely to the side, is harder. This is as the field lines of the small diodes follow rather a radial than a parallel geometry. As a consequence, the depletion depth reached scales rather $\sqrt[n]{U_{\text {depl }}}$. The precise value of $n$ is so far not established. Given the complex geometry of CPS with small diodes, non-trivial findings have to be expected. As argued more below in this work, first observations with MIMOSA-series prototypes favour $n \approx 3$.

From the experimental point of view, one has to consider that the classical $\mathrm{C}-\mathrm{V}$ test for full depletion, as known from planar detectors, might not be reliable. This is as the underlying assumption of a flat junction is not fulfilled. Mapping out the active volume with an ${ }^{55} \mathrm{Fe}$-source (see Sec. 4.3.4 appears as a reasonable complement for estimating the depleted volume. Useful additional information may be obtained from the leakage currents of mildly neutron irradiated samples (see Sec. 6.1.2 [78]. In any case, one has to consider that a saturation of the above mentioned parameters only indicate that the depleted volume is not further extended with increasing depletion voltage. This does not necessarily mean that a full depletion is reached.

The preliminary results presented below were obtained with the prototypes MIMOSA34, PIPPER-2, which were made at the IPHC Strasbourg. They rely on the Tower/Jazz $180 \mathrm{~nm}$ quad-well CMOS process, which provides a high resistivity epitaxial layer. The concept of applying a high voltage to the charge collection diode despite of the voltage limitations of the CMOS process accounts for the fact that this limitation is mostly defined by the vulnerable transistor gates while other components are more voltage tolerant.

The schematics developed based on this assumption is shown in Fig. 23. The sensors contain a standard SB-sensing element, which relies on a biasing diode and the usual charge 


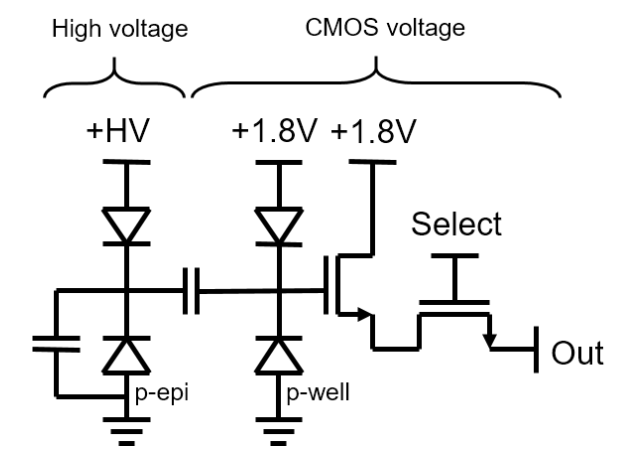

Figure 23: Schematics of the AC-coupled pixel of PIPPER-2.

collection diode. The biasing diode is contacted exclusively by metal lines, which are isolated by massive and voltage tolerant $\mathrm{SiO}_{2}$. The forward biased biasing diode as such does not require a particular voltage tolerance as the potential drop of this component with respect to the surrounding n-well of the charge collection diode remains unchanged. The structure of the charge collection diode was however modified in the PIPPER prototypes by extending the opening of the p-well around the n-well implantation of the diode. Such, a ring with low p-epi doing is created, which increases the punch through limit of the diode. The maximum voltage allowed increased successively and reached about $40 \mathrm{~V}$ for the PIPPER-2 sensor. The high voltage sensing element is separated from the low voltage pre-amplifier by means of ACcoupling. This is realized with a high voltage tolerant capacitor. The DC potential of the low voltage side of the capacitor is set by another pair of forward and backward biased diodes, which serve as a kind of voltage divider.

\subsubsection{Observations on MIMOSA-34}

A first study on this kind of pixel was carried out with the imager MIMOSA-34 HR30. The sensor based on a customized wafer, which shows a doping of about $P \approx 3 \times 10^{11} / \mathrm{cm}^{3}$ with a slight minimum at a depth of $18 \mu \mathrm{m}$. Below this minimum, the doping increases rapidly until it reaches $P \approx 10^{19} / \mathrm{cm}^{3}$ at a depth of $28 \mu \mathrm{m}$. One pixel of MIMOSA-34 (P13) is suited to apply depletion voltages of up to $\sim 9 \mathrm{~V}$. Its pitch is $33 \mu \mathrm{m}$ in both dimensions the octagonal $\mathrm{N}$-Well forming the collection diode features a "radius" of $r=1.5 \mu \mathrm{m}$. The distance between this N-Well and the P-Well layer covering the remaining pixel amounts $0.4 \mu \mathrm{m}$.

The depleted volume of the pixels of MIMOSA-34 HR30 was mapped out with an ${ }^{55} \mathrm{Fe}-$ amplitude spectra as introduced in Sec. 4.3.4 and computed based on Eq. 11. A pixel volume of $V_{\text {pixel }}=33 \times 33 \times 28 \mu \mathrm{m}^{3}$ was anticipated based on the known design values of the sensor. As the attenuation of the X-rays in silicon is not negligible, a correction factor was applied to $N_{\text {all }}$. This was not done for $N_{\text {depl }}$ because this zone is comparably shallow (which reduces the attenuation effect) and that a precise knowledge on its geometry would be needed to find the appropriate factor. This is assumed to create a $\sim 10 \%$ bias toward a too high depleted volume $V_{d e p l}$. An approximate depletion depth $r_{d}$ was obtained by assuming that the depleted 


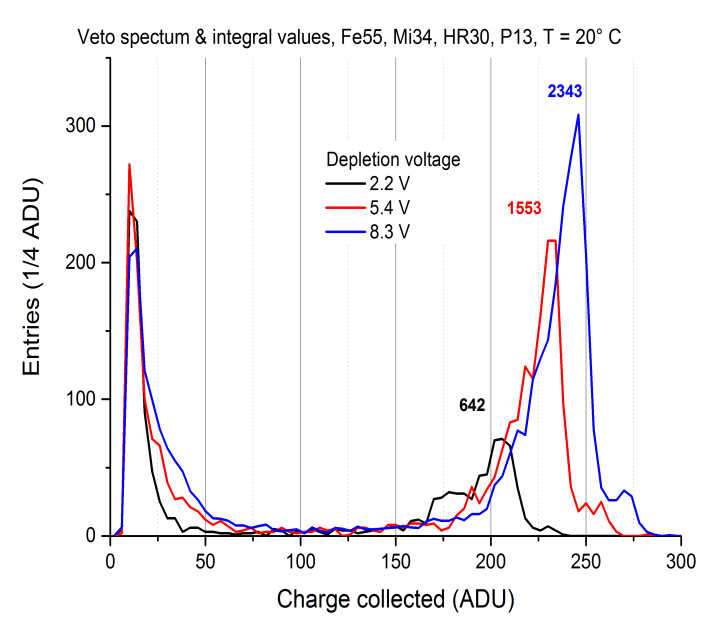

Figure 24: Amplitude spectrum indicating the response of MIMOSA-34 HR30 to $5.9 \mathrm{keV}$ photons. Clusters showing charge sharing were removed in order to highlight hits occurring in the depleted zone of the pixel.

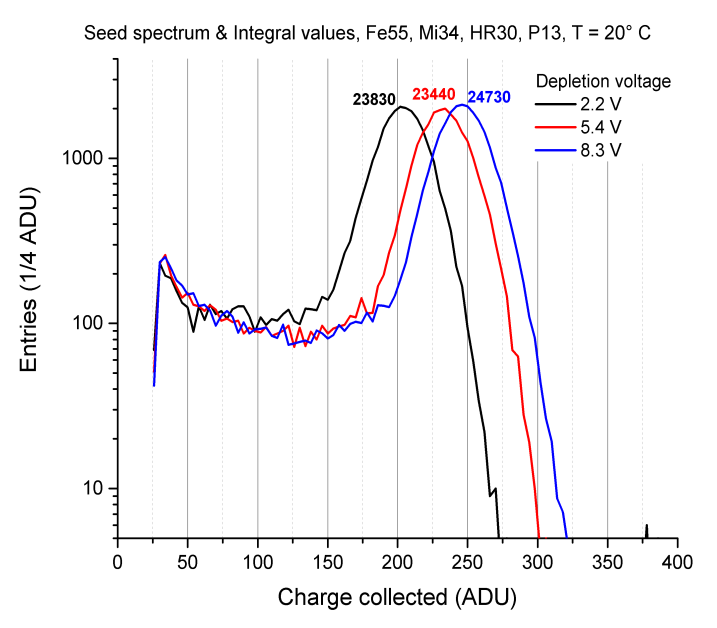

Figure 25: Amplitude spectrum indicating the response of MIMOSA-34 HR30 to $5.9 \mathrm{keV}$ photons. The charge of all pixels of the cluster was added.

volume is hemispheric and computing the radius of this hemisphere according to:

$$
r_{d} \approx \sqrt[3]{\frac{3}{2 \pi} V_{d e p l}}
$$

According to TCAD [74] simulations, this assumption of a hemispheric depleted volume is only approximatively fulfilled [77].

Examples for the ${ }^{55} \mathrm{Fe}$ amplitude spectra recorded are shown in Fig. 24 and Fig. 25 , which show the seed pixel spectrum and a spectrum summing up the signals of all pixels respectively. Note that the seed spectrum was modified by rejecting all clusters, which show charge sharing. This rejects essentially the charge collection peak of the seed spectrum and highlights the calibration peak as required for this study. One observes that the X-position of the calibration peak of the seed pixel spectrum is moving toward higher values with increasing depletion voltage. This might be interpreted as a reduction of the pixel capacity as expected for charge collection diodes with an increasing depleted volume. Note, however, that this capacity is a priori not suited to measure for the size of the depleted zone in a quantitative way. This is as the capacity of elements other than the charge collection diode cannot be neglected. Besides of being displaced, the calibration peaks gain in number of entries while the charge collection peaks shown in Fig. 25 remain essentially unchanged. This is as the depleted volume of the charge collection diode increases while the total volume of the pixel as defined by the pitch and the thickness of the epitaxial layer does not.

The results for the are displayed in Fig. 26, which shows also the depletion depth of the collection diode according to TCAD simulations of the device. The results were fitted with a power law according to

$$
r_{d}=A \cdot \sqrt[n]{U_{d e p l}}
$$




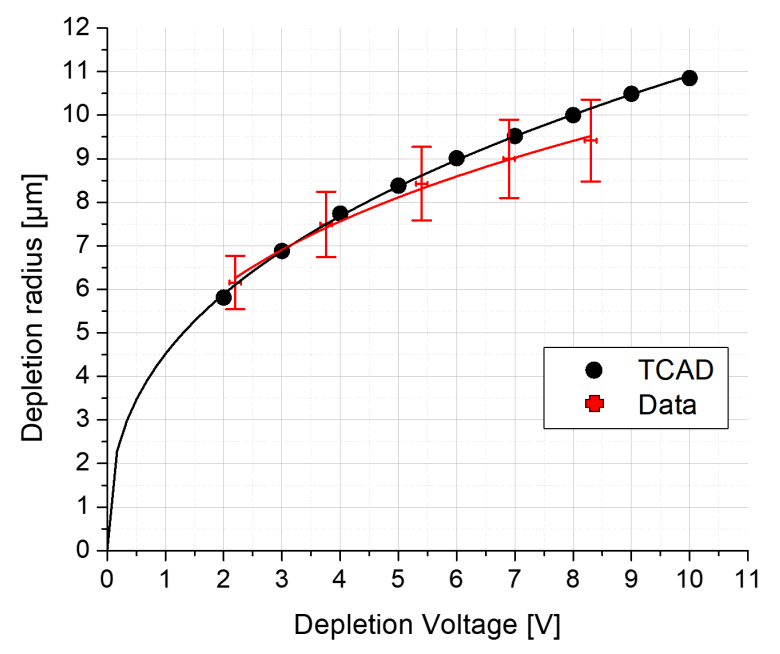

Figure 26: Depletion radius of the AC-coupled pixel of MIMOSA-34 as a function of the depletion voltage. Measured data from [73], and the results of TCAD simulations from [77] are compared and fitted with a power function. See text.

A result of $n=2.6$ and $n=3.2$ is found for the simulated and the measured depletion depth. Given the numerous uncertainties and approximations of the analysis, both values are considered as compatible with the value $n=3$, which would be expected for an abrupt and tiny hemispherical junction. Note that this result holds for depletion zones, which do neither touch the lower limit of the epitaxial layer nor the deplete zone of a neighbouring pixel. Moreover, one should mention that the absolute size of the depleted zone as defined by $A$ is unexpectedly small.

\subsubsection{Observations of PIPPER-2}

A second round of measurements was carried out with the PIPPER-2 prototype, which featured pixels with a pitch of $22 \times 22 \mu \mathrm{m}^{2}$ and a minimum integration time of $12.8 \mu \mathrm{s}$. The prototype was realized in a Tower/Jazz $180 \mathrm{~nm}$ with $18 \mu \mathrm{m}$ high resistivity epitaxial layer. With respect to MIMOSA-34, this chip was modified by extending the radius of the diode but more importantly by choosing a substantially more sizeable opening of the p-well surrounding it. As reported in [79], the chip operated at depletion voltages of up to $40 \mathrm{~V}$. Based on ${ }^{55} \mathrm{Fe}$ amplitude spectra and TCAD simulations, it is concluded that the chip becomes fully depleted once the depletion voltage exceeds about $15 \mathrm{~V}$. The simulation depth reported is $\sim 12 \mu \mathrm{m}$, which corresponds to about $66 \%$ of the volume of the epitaxial layer. Based on TCAD simulations, the authors argue that a depletion layer is formed in this epitaxial layer. According to this, a $\sim 6 \mu \mathrm{m}$ thick layer within the epitaxial layer remains non-depleted. This layer may be identified with the border of the lowly doped region of this epitaxial layer toward the highly doped substrate. This area is known to show a sizeable and steadily increasing doping toward the substrate, which makes it hard to deplete.

A first pilot study on the tolerance of PIPPER-2 to non-ionizing radiation was carried out 


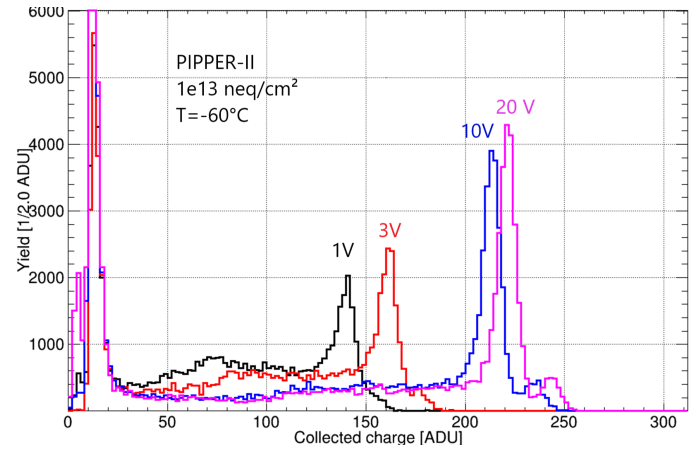

(a) ${ }^{55} \mathrm{Fe}$ spectrum of a PIPPER-II prototype, which was irradiated with $10^{13} \mathrm{n}_{\mathrm{eq}} / \mathrm{cm}^{2}$ as a function of the bias voltage applied.

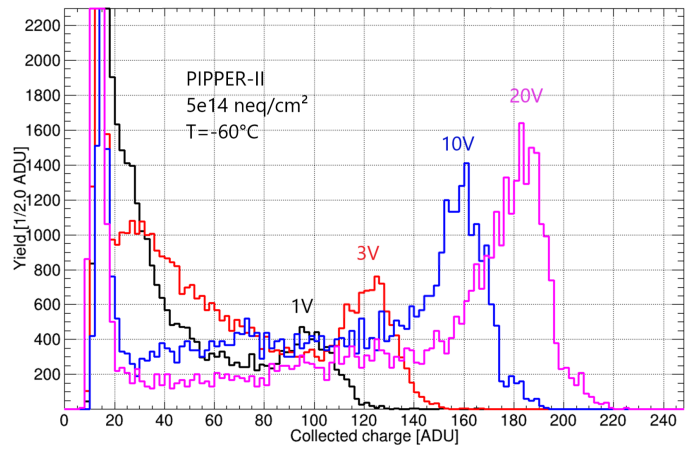

(b) ${ }^{55} \mathrm{Fe}$ spectrum of a PIPPER-II prototype, which was irradiated with $5 \times 10^{14} \mathrm{n}_{\mathrm{eq}} / \mathrm{cm}^{2}$ as a function of the depletion voltage applied.

Figure 27: Preliminary results on the charge collection efficiency of the PIPPER-II prototype as a function of the depletion voltage and radiation dose. Mind the scale. From [78].

with sensors being irradiated to $10^{13} \mathrm{n}_{\mathrm{eq}} / \mathrm{cm}^{2}$ and $5 \times 10^{14} \mathrm{n}_{\mathrm{eq}} / \mathrm{cm}^{2}$. The thickness of the depleted zone of the sensor was mapped out with a ${ }^{55} \mathrm{Fe}$ source. Moreover, a ${ }^{109} \mathrm{Cd}$ source was used. This choice reflects the fact that the $22 \mathrm{keV}$ photons of this source have a higher penetration power in $\mathrm{Si}$ than the $5.9 \mathrm{keV}$ photons of ${ }^{55} \mathrm{Fe}$. Therefore, no correction for the attenuation of the X-rays within the epitaxial layer is required.

An ${ }^{55} \mathrm{Fe}$ seed pixel spectrum is shown in Fig. 27 for sensors irradiated to both radiation level. As expected from the above mentioned literature, the charge collection peak does mostly vanish after applying a bias voltage of $10 \mathrm{~V}$ to the diode and no significant modification of the spectrum is observed for the sensor irradiated to $10^{13} \mathrm{n}_{\mathrm{eq}} / \mathrm{cm}^{2}$. This holds to a reduced extend for sensor exposed to $5 \times 10^{14} \mathrm{n}_{\mathrm{eq}} / \mathrm{cm}^{2}$.

Results from measurements of the leakage current of PIPPER-2 as displayed in Fig. 28. Fig. 28a indicates the absolute leakage current per pixel as measured with an ammeter from at the biasing line of the diodes. Note that the results are somewhat biased by the fact that PIPPER-2 integrates three slightly different pixel structures, among which one has four charge collection diodes. As those pixels were connected in parallel, the summed leakage current of all three pixel flavours is shown. This should however have few effect on the results as soon as full depletion is reached. One observes that the leakage current per diode reaches values of $\sim 6 \mathrm{pA}$ after a dose of $10^{13} \mathrm{n}_{\mathrm{eq}} / \mathrm{cm}^{2}$ and $120 \mathrm{fA}$ after a dose of $5 \times 10^{15} \mathrm{n}_{\mathrm{eq}} / \mathrm{cm}^{2}$. Due to the higher depleted volume, those leakage currents are huge as compared to the leakage currents observed with irradiated, non depleted sensors. Therefore, one expects serious issues with shot noise and signal clearing. Indeed, the sensors had to be cooled to temperatures as low as $-60{ }^{\circ} \mathrm{C}$ before reasonable tests could be carried out. Despite this low temperature and the fast integration time of the device, signal clearing remained an issue. A remarkable observation is made once one computes the thickness of the depletion layer from the leakage currents in accordance with Eq. 2 (see Fig. 28b). While the highly irradiated sensor reproduces the previously reported thickness of the depleted layer, the indication of the sensor irradiated to $10^{13} \mathrm{n}_{\mathrm{eq}} / \mathrm{cm}^{2}$ overshoots and converges to an indicated thickness of $34 \mu \mathrm{m}$. 


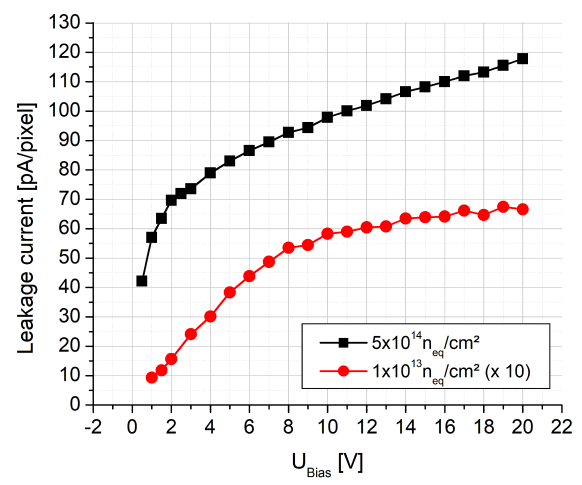

(a) Combined leakage current of the three pixel matrices of PIPPER-2 at a temperature of $20{ }^{\circ} \mathrm{C}$. The leakage current of the sensor irradiated with $10^{13} \mathrm{n}_{\mathrm{eq}} / \mathrm{cm}^{2}$ was multiplied with a factor of 10 for clearity. See text.

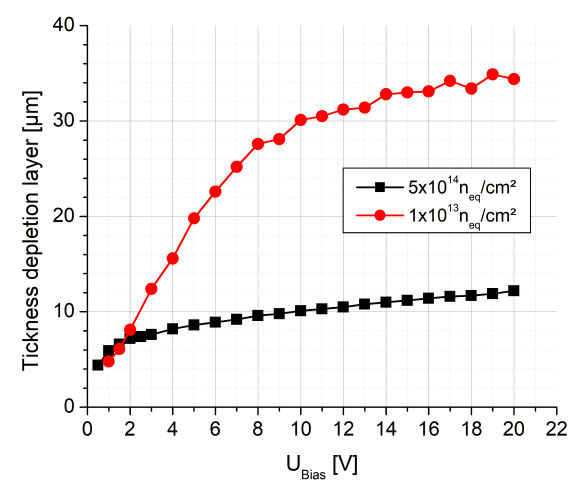

(b) Thickness of the depleted layer of PIPPER-2 as indicated by the leakage current.

Figure 28: Preliminary results on the leakage current of neutron irradiated PIPPER-2 sensors.

Data from [78].

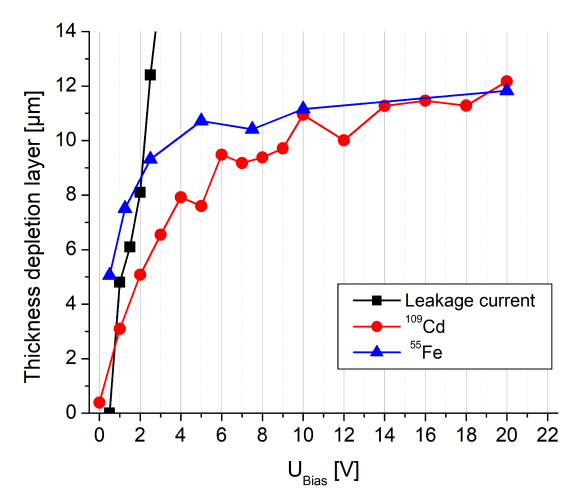

(a) Thickness of the depletion layer of PIPPER-2 as function of the bias voltage for a dose of $10^{13} \mathrm{n}_{\mathrm{eq}} / \mathrm{cm}^{2}$.

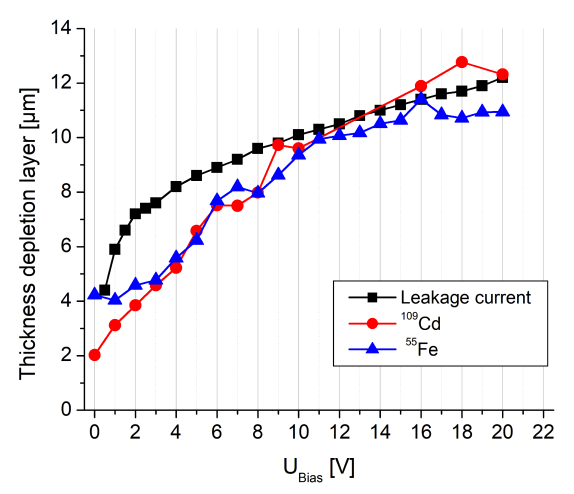

(b) Thickness of the depletion layer of PIPPER-2 as function of the bias voltage for a dose of $5 \times 10^{14} \mathrm{n}_{\mathrm{eq}} / \mathrm{cm}^{2}$.

Figure 29: Preliminary results on the thickness of the depletion layer of neutron irradiated PIPPER-2 sensors as obtained from amplitude spectrum measurements. Data from [78]. 
Preliminary results on the thickness of the depleted layers as obtained from mapping the sensor with a ${ }^{109} \mathrm{Cd}$ and a ${ }^{55} \mathrm{Fe}$ source are shown in Fig. 29. The plots map the estimated thickness of the depleted layer as a function of the voltage applied to the diode. The indications of the leakage current measurements are shown for comparison. The results displayed in Fig. $29 \mathrm{a}$ hold for the sensor irradiated with $10^{13} \mathrm{n}_{\mathrm{eq}} / \mathrm{cm}^{2}$. The amplitude spectra indicate a layer thickness of $\sim 12 \mu \mathrm{m}$ at a voltage of $20 \mathrm{~V}$, which can be considered as full depletion within the known limits of the sensor. The results match the expectation and contrast the results of the leakage current. The latter is thus considered as biased by a non-identified source of current.

For the sensor irradiated to $5 \times 10^{14} \mathrm{n}_{\mathrm{eq}} / \mathrm{cm}^{2}$, one observes all three measurements to show similar indications as soon as a reasonable degree of depletion is reached. The indication suggests that the sensor depletes slower than the $10^{13} \mathrm{n}_{\mathrm{eq}} / \mathrm{cm}^{2}$ sensor but a full depletion is reached once the highest voltage is applied.

It should be mentioned that the sensor irradiated with $10^{13} \mathrm{n}_{\mathrm{eq}} / \mathrm{cm}^{2}$ shows a noise of 23 electrons at a temperature of $-55{ }^{\circ} \mathrm{C}$ and an integration time of $12.5 \mu \mathrm{s}$. The most probable charge collected from the $\beta$-rays of a ${ }^{90} \mathrm{Sr}$ source was found to amount $1290 \mathrm{e}$ at a depletion voltage of $20 \mathrm{~V}$, which turns into a $\mathrm{S} / \mathrm{N}$ of 55 . For the sensor irradiated with $5 \times 10^{14} \mathrm{n}_{\mathrm{eq}} / \mathrm{cm}^{2}$, a noise of 27 electrons and a most probable charge of $\gtrsim 860 e$ was seen. The remaining $\mathrm{S} / \mathrm{N}$ of 31 and the fact that a full depletion appears reached once a sufficient voltage is applied suggests that the sensor did withstand the radiation load without loss of sensitivity for minimum ionizing particles. However, it remains to be rigorously shown that the rule " $>99 \%$ detection efficiency if $\mathrm{S} / \mathrm{N}>15$ " holds also for highly irradiated, depleted sensors. 


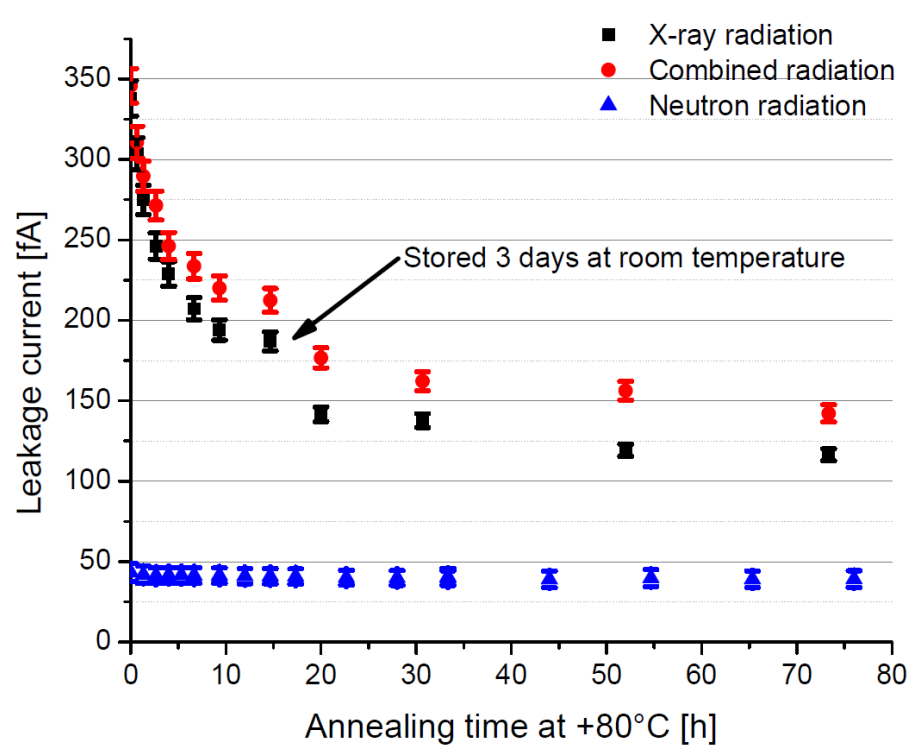

Figure 30: Leakage current of irradiated sensors as a function of the annealing time. See text. From [80].

\section{Thermal Annealing}

Both, ionizing and non-ionizing radiation damage are known to be modified by annealing. During this process, a part of the microscopic defects are recovered. In case of surface damage, this may e.g. happen in case a hole trapped at the interface between $\mathrm{Si}$ and $\mathrm{SiO}_{2}$ is filled by a thermally excited electron. In the case of bulk damage, an interstitial atom may for example recombine with a vacancy in the crystal. On the other hand, some defects may bind to more complex defect molecules, which are sometimes more damaging than the initial defect. In this case, the annealing generates additional macroscopic damage and is referred to as reverse annealing [22].

In a first study on annealing in CPS [40], charge collection diodes and pixels implemented in a $0.25 \mu \mathrm{m}$ CMOS process were irradiated with $800 \mathrm{krad}$. During half the irradiation, the sensor was not powered. The leakage currents were found to increase by a factor of 200 after irradiation and decreased by a factor of three after three weeks of room temperature annealing. 24 hours of forced annealing at a temperature of $100^{\circ} \mathrm{C}$ reduced the leakage currents by another factor of five.

A more systematic study was carried out with a CPS named MIMOSA-19, which based on an AMS 0.35 Opto process with $12 \mu \mathrm{m}$ pixel pitch and a $14 \mu \mathrm{m}$ low resistivity epitaxial layer [80, 81. The L-shaped diodes of the sensor have a surface of $40 \mu \mathrm{m}^{2}$ and are not hardened against ionizing radiation damage. The sensor was irradiated with $\sim 1 \mathrm{MeV}$ neutrons to a dose of $1.95 \mathrm{n}_{\mathrm{eq}} / \mathrm{cm}^{2}$ and with soft X-rays to a dose of $200 \mathrm{krad}$. One sensor was exposed consecutively to both radiation doses.

During and after the neutron irradiation, the sensors were stored for few weeks at room temperature until they were bonded and ready for test. Hereafter, their charge collection efficiency, gain, leakage current and noise were studied. This test was repeated after one year of room temperature storage. According to the test results, no significant modification of the charge collection efficiency and of the sensor gain were observed (within $\sim 5 \%$ uncertainty). Concerning the leakage current and the noise, a slight but non-significant trend 
toward beneficial annealing was observed.

Once the measurements were concluded, a virgin sensor and a sensor, which was previously irradiated with neutrons, were irradiated with $200 \mathrm{krad}$ soft X-rays. The irradiation was done at room temperature and the properties of the irradiated sensors was measured two hours after irradiation. As expected, the ionizing dose caused a strong increase of the leakage currents $I_{L}$ in both chips. The sensor, which was exposed to both, X-rays and neutrons, showed an $I_{L}$, which amounted about the sum of the leakage currents observed at sensors suffering from the individual radiation damages.

During the following $280 \mathrm{~h}$, the pixels were stored at room temperature and leakage current of their pixels was monitored. About $20 \%$ of the total current was observed to vanish according to an exponential decay with a time constant of about $90 \pm 50 \mathrm{~h}$. Both sensors showed a similar behaviour within error bars, which suggests that the annealing acted on the surface damage. After the room temperature annealing went into saturation, a forced annealing was performed by heating the sensors to $T=80^{\circ} \mathrm{C}$. As illustrated in Fig. 30, the leakage currents of the $\mathrm{X}$-ray irradiated sensors was reduced by more than a factor of two. The annealing procedure was also applied to a sensor, which was irradiated with $1.95 \mathrm{n}_{\mathrm{eq}} / \mathrm{cm}^{2}$ neutrons only. This sensor shows a significant $9 \%$ decrease of the leakage currents after annealing. Moreover, a significant $5 \%$ improvement of the charge collection efficiency are reported.

Over all, one may conclude that thermal annealing is suited to reduce the leakage currents as caused by ionizing radiation. Beneficial effects on bulk damage have been seen but they are too small to be of practical relevance. More importantly, no reverse annealing was observed for sensors with low resistivity epitaxial layer. Note, that no statement is made on potential reverse annealing of sensors with high resistivity epitaxial layer. 


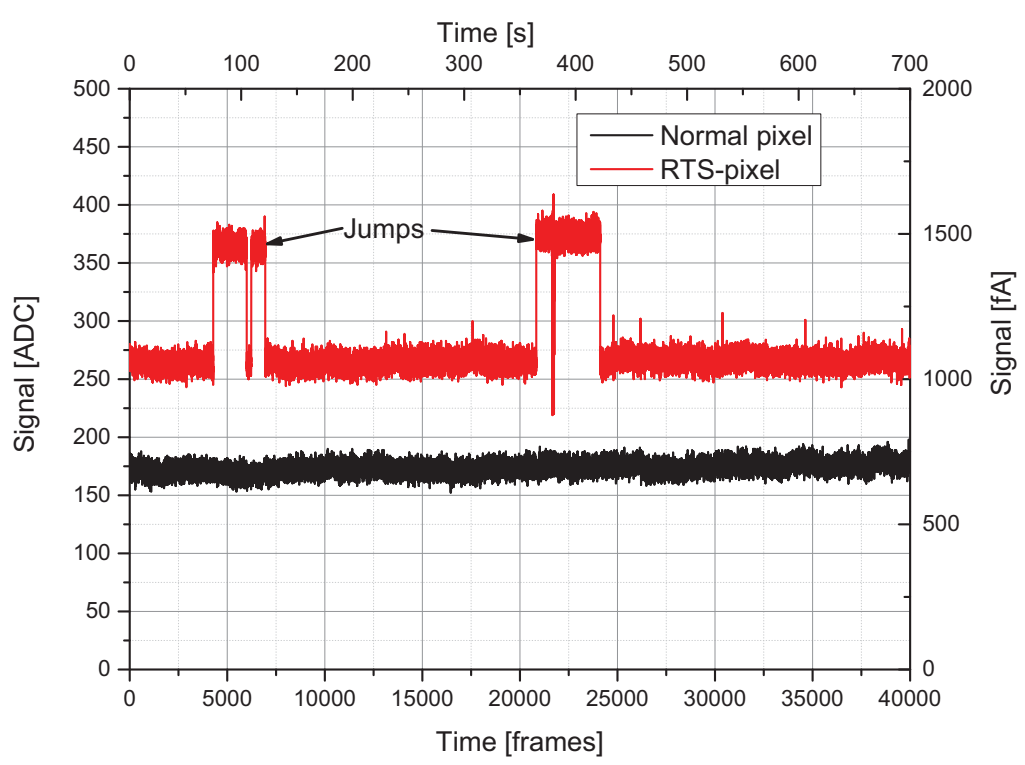

Figure 31: Random Telegraph Signal (RTS): The upper (red) signal shows the leakage current of a 3T-pixel with RTS behaviour whereas the lower (black) line corresponds to the output of a stable pixel without RTS. Once the amplitude of the "jumps" exceed the threshold of the detector, numerous consecutive false hits are indicated by the RTS-pixel. From [82].

\section{Specific radiation damage effects}

\subsection{Random Telegraph Signal}

\subsubsection{Introduction}

As shown in Fig. 31, the leakage current of reverse biased diodes may be found modulated by a significant rectangular signal with substantial amplitude. This signal is referred to as Random Telegraph Signal (RTS). It is likely caused by a system of two defects, which are located in or nearby the PN-junction. The first defect may absorb or emit an electron and thus change its electric state. The related electric field switches a current source, which forms the second element of the system. As the switching electron may only be present or absent, the current is modulated between two well defined levels and superimposes the leakage currents caused by other sources. RTS with more than two levels was observed and is usually explained as a superposition of multiple RTS-sources.

In CPS, RTS may modulate the leakage current of the collection diodes rather significantly. Moreover, the input transistor of the on-pixel pre-amplifier may be affected. In the latter case, the transconductance of the transistor is modulated. Both modulations may generate signatures, which can be misinterpreted as particle impacts. Therefore, RTS is suited to transform normal pixels into "hot" pixels.

Well designed CPS are mostly free of RTS. After exposing the devices to radiation, the number of pixels showing RTS increases dramatically. Therefore, RTS can be considered as 
a radiation damage in CPS. Initially, it was believed that RTS is caused exclusively by nonionizing radiation. However, recent studies revealed that RTS may also be caused by ionizing radiation.

\subsubsection{RTS in the leakage current of charge collection diodes}

8.1.2.1 Parametrization, properties and detection of RTS RTS in the leakage current is a property of the individual charge collection diode. The probability of a pixel to show RTS is not correlated to the state of the neighbouring pixels [83], but increases for diodes showing a high absolute leakage current [84]. For neutron irradiated sensors, this probability is reported to scale with the NIEL (see for example [84, 85, 86]). The amplitude of the RTS is reduced once the depletion voltage of affected diodes is reduced [87]. In case, this feature is related to the strength of the electric field in the diode, HR-sensors might be less vulnerable to RTS than LR-sensors, which remains to be confirmed.

Different strategies including visual inspection of the data have been used by different authors to state whether a pixel is affected by RTS. Their common feature is that RTS can be recognized only once its amplitude $I_{\mathrm{RTS}}$ exceeds the thermal noise of the individual pixel $Q_{\text {Noise }}$ significantly. Therefore, any recognition concept ends up in comparing $I_{\text {RTS }}$ and $Q_{\text {Noise }}$ in a more or less elaborated way. This is typically done by converting the RTS-signal into the related integrated charge. Therefore, a pixel may be considered to be affected by RTS if:

$$
I_{\mathrm{RTS}}>N_{t} \cdot \frac{Q_{\text {Noise }}}{t_{\text {int }}}
$$

Here, $t_{i n t}$ is the integration time of the pixel and $N_{t}$ represents a threshold constant. For CPS used for charged particle tracking, it is reasonable to identify $N_{t}$ with the particle identification threshold. In the following, it will be of use to define the RTS recognition threshold $I_{\mathrm{N}_{\mathrm{RTS}}}$ as:

$$
I_{\mathrm{N}_{\mathrm{RTS}}}\left(t_{\mathrm{int}}, T\right)=N_{t} \cdot \frac{Q_{\text {Noise }}}{t_{\mathrm{int}}}
$$

This definition is obviously ambiguous as the threshold changes as a function of the integration time and as both, $I_{\mathrm{RTS}}$ and $Q_{\text {Noise }}$ may depend on the temperature. This unresolved issue complicates the comparison of data provided by the different pioneering studies [84, 87, 88, 89].

A very careful and recent study on the nature of RTS in diodes is found in [85]. As a strong point, this paper relies on a comprehensive detection concept for RTS, which turns into a very low $I_{\mathrm{N}_{\mathrm{RTS}}}\left(t_{\mathrm{int}}, T\right)$. According to this study, the probability to identify a pixel with a certain RTS amplitude is empirically found to be described by the distribution:

$$
P\left(I_{\mathrm{RTS}}\right)=k \cdot \lambda \cdot \exp \left(-\lambda \cdot I_{\mathrm{RTS}}\right)
$$

Here, $k$ is a normalisation constant and $1 / \lambda$ is given with $1 / \lambda \approx 110 \mathrm{e} / \mathrm{s}$ for a sensor irradiated with 10 Gy X-rays and with $1 / \lambda \approx 1200 \mathrm{e} / \mathrm{s}$ for a sensor irradiated with $1.8 \times 10 \mathrm{n}$ eq $/ \mathrm{cm}^{2}$ neutrons. A sensor, which was irradiated with protons to the sum of both doses showed an RTS amplitude distribution, which can be interpreted as the sum of of the individual distributions for ionizing and non-ionizing irradiation. The very low amplitude of the RTS as caused by surface damage explains why this RTS was initially not observed. 


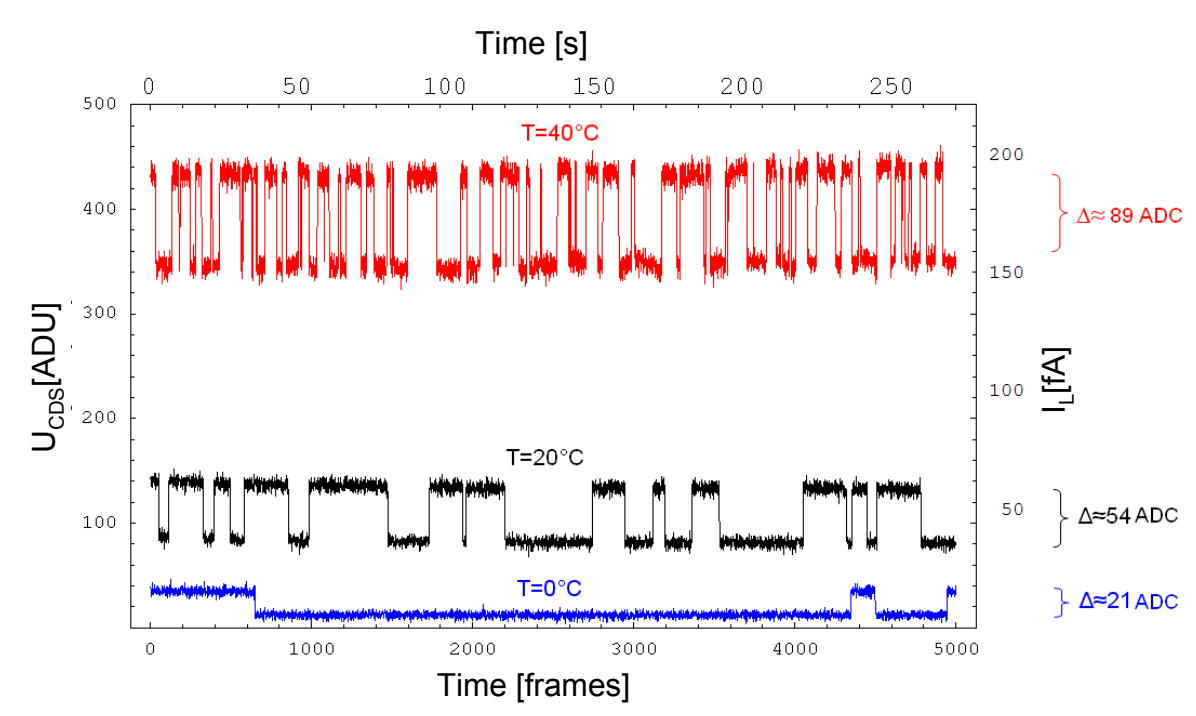

Figure 32: Temperature dependence of the RTS-amplitude and the RTS-frequency in a selected 3T-pixel of MIMOSA-19. From [89].

As illustrated in Fig. 32 , the amplitude of the RTS is found to scale with temperature. A related parametrization is given in [85] with:

$$
I_{R T S} \propto \exp \left(-\frac{E_{a}}{k_{B} T}\right)
$$

where $I_{R T S}$ is the transition maximum amplitude and $E_{a}$ is the activation energy. This energy is a property of the individual pixel and seems to vary over a significant range around the mid-gap energy.

The width of the RTS pulses spans a rather long range. Average pulse lengths faster than a fraction of a second are observed as much as pixels, which show RTS after several tens of minutes of inactivity. For CPS, which were irradiated with $11.7-59 \mathrm{MeV}$ protons, the probability of a pulse with a time $t_{p u l s e}$ to occur is empirically given [84] with:

$$
P\left(t_{\text {pulse }}\right)=C_{N} \cdot \exp \left(-\frac{t_{\text {pulse }}}{\tau}\right)
$$

Here, $C_{N}$ is a normalisation constant and $\tau$ is given with:

$$
\tau^{-1} \propto \exp \left(-\frac{E_{a}}{k_{B} T}\right)
$$

For the upper state of the RTS signal, the activation energy is reported to amount $E_{a c t, u}=0.58 \mathrm{eV}$ for temperatures between $23^{\circ} \mathrm{C}$ and $57^{\circ} \mathrm{C}$. For the lower state, $E_{a c t, l}=0.61 \mathrm{eV}$. Note that this parametrization is not suited to describe pixels exhibiting more than two states [85]. 


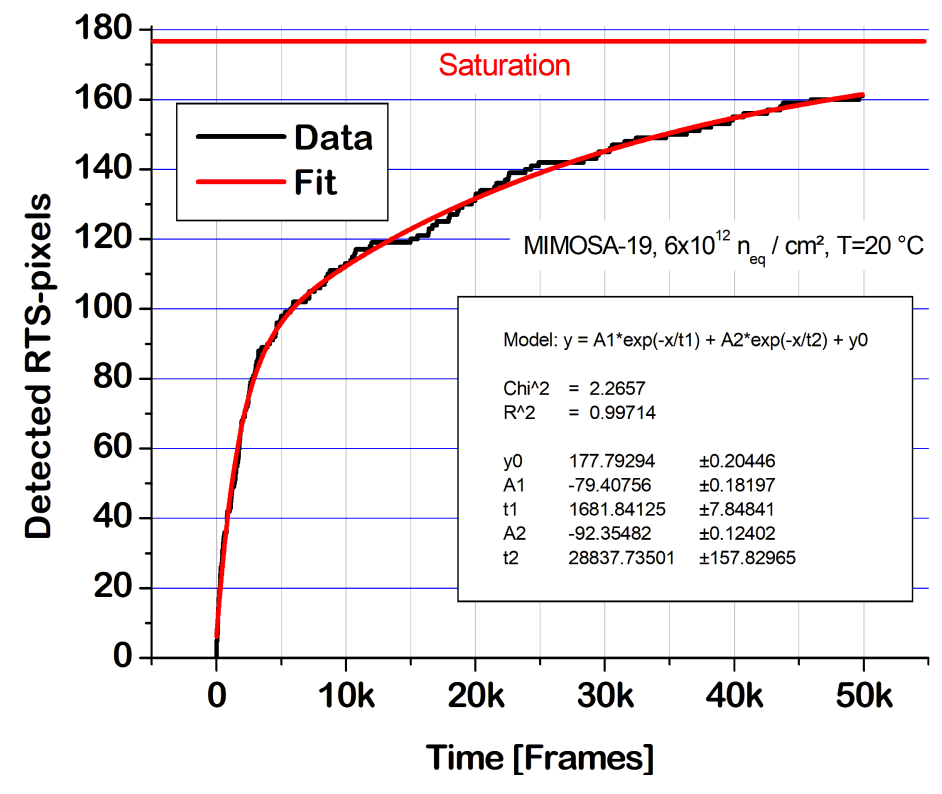

Figure 33: Number of identified RTS pixels as function of the observation time (MIMOSA-19, $T=20{ }^{\circ} \mathrm{C}, \Phi=6 \times$ $\left.10^{12} \mathrm{n}_{\mathrm{eq}} / \mathrm{cm}^{2}, I_{\mathrm{N}_{\mathrm{RTS}}}=6.7 \mathrm{fA}\right)$. The saturation value was obtained by fitting the data with the function displayed in the inlet box and by extrapolating the function obtained to infinity. After 83 .

8.1.2.2 Number of RTS-pixel as a function of radiation doses Most studies on the probability of obtaining an RTS pixel have been carried out in the field of optical imaging under space conditions. This application requires rather long integration times, which turns into a low $I_{\mathrm{N}_{\mathrm{RTS}}}\left(t_{\mathrm{int}}, T\right)$. In [85], CPS imagers with $128 \times 128$ pixels of $10 \mu \mathrm{m}$ pitch, which were manufactures in a $0.18 \mu \mathrm{m}$ CMOS process, were used. The sensors were irradiated with ionizing and non-ionizing radiation and operated hereafter at a temperature of $T=22^{\circ} \mathrm{C}$. RTS was detected by scanning the leakage currents with edge recognition techniques. This provided a very low threshold, which estimated to amount $I_{\mathrm{N}_{\mathrm{RTS}}}\left(t_{\mathrm{int}}, T\right) \approx 10^{-17} \mathrm{~A}$. The number of pixels affected by RTS is observed to scale linearly with the non-ionizing dose and to reach $\sim 70 \%$ of all pixels at the highest dose applied $(365 \mathrm{TeV} / \mathrm{g}$, which is equivalent to $\left.1.7 \times 10^{11} \mathrm{n}_{\mathrm{eq}} / \mathrm{cm}^{2}\right)$. For the ionizing dose, the number of RTS-pixels increases rapidly as well but the scaling shows a signature of a saturation. However, $100 \%$ of all pixels are reported to show RTS after a dose of $100 \mathrm{~Gy}=10 \mathrm{krad}$. As both doses are rather moderate as compared to the requirements of moderate detectors of heavy-ion and particle physics, one may conclude that CPS operated in radiation fields will show RTS in all pixels at some point.

Similar observations were made in an elder study carried out with the STAR-250 CMOS image sensor [84]. This sensor hosted $512 \times 512$ pixels with $25 \mu \mathrm{m}$ pitch with four diodes per pixel. It was manufactured in a $0.5 \mu \mathrm{m}$ CMOS process. RTS-pixels were defined as pixels, which show a RTS-amplitude/dark current ratio above $2.5 \%$ and an RTS amplitude of $I_{\mathrm{N}_{\mathrm{RTS}}}\left(t_{\mathrm{int}}, T\right)=1.25 \cdot 10^{-16}$ A per pixel at an operation temperature ranging from $23^{\circ} \mathrm{C}$ to $57^{\circ} \mathrm{C}$. The study revealed that RTS tends to occur preferably in pixels with high leakage currents and a low activation energy of this leakage current. No RTS caused by ionizing radiation was observed, likely due to missing experimental senstivity. Again, a rapid increase of the number of RTS-pixels as a function of non-ionizing radiation dose is observed.

While both of the previous studies aimed for a use of CPS for optical imaging, dedicated issues of RTS in CPS for charged particle detection were addressed in [83, 89]. Here, a pixel was 

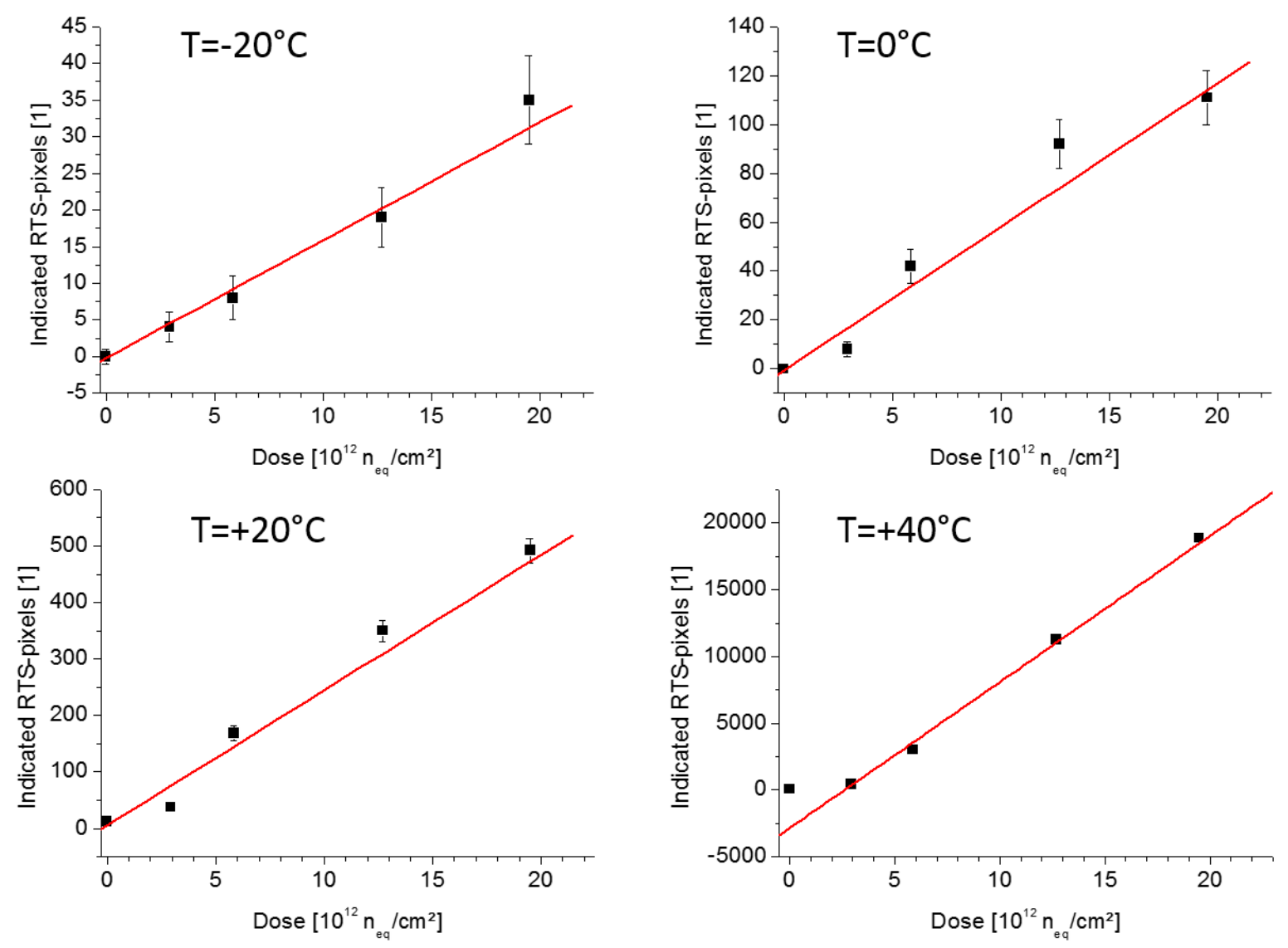

Figure 34: Number of indicated RTS-pixels in MIMOSA-19 as function of the temperature and the $1 \mathrm{MeV}$ neutron dose with linear fit. Data from [89].

considered as RTS pixel if it generated at least one signature of a upper signal of $0.5 \mathrm{~s}$ length during a measurement time of 45 minutes. An RTS recognition threshold of $I_{\mathrm{N}_{\mathrm{RTS}}}\left(t_{\mathrm{int}}, T\right)=$ $6.7 \mathrm{fA}$ ( 150 e per readout cycle of $3.6 \mathrm{~ms}$ ) was chosen, which was motivated by the amplitude level required to generate false hit indications. Based on this definition, the number of RTS pixels of $\sim 1 \mathrm{MeV}$ neutron irradiated MIMOSA-19 sensors $(192 \times 192$ 3T-pixels, low resistivity epitaxial layer, serial analogue output with $t_{\text {int }}=3.6 \mathrm{~ms}$ for $f_{c l k}=10 \mathrm{MHz}$ ) was measured as function of the temperature and the radiation dose. The measurement was carried out for about 45 minutes and each of the $196 \times 196=38416$ pixels was scanned for RTS signatures. As displayed in Fig. 33, the number of recognized RTS pixels was found not to saturate during this observation time. Therefore, the data was fitted and the fit function was extrapolated toward an infinite observation time. Thanks to the exponential nature of the fit function, this turned into a well defined saturation value on the number of RTS pixels. It was found that the data is described by a double-exponential decay function. This suggests that two independent processes with different time scale might be involved.

As reported by the previously mentioned studies, one finds the number of affected pixels to depend on the temperature but to scale linearly with the radiation dose. This implies that 


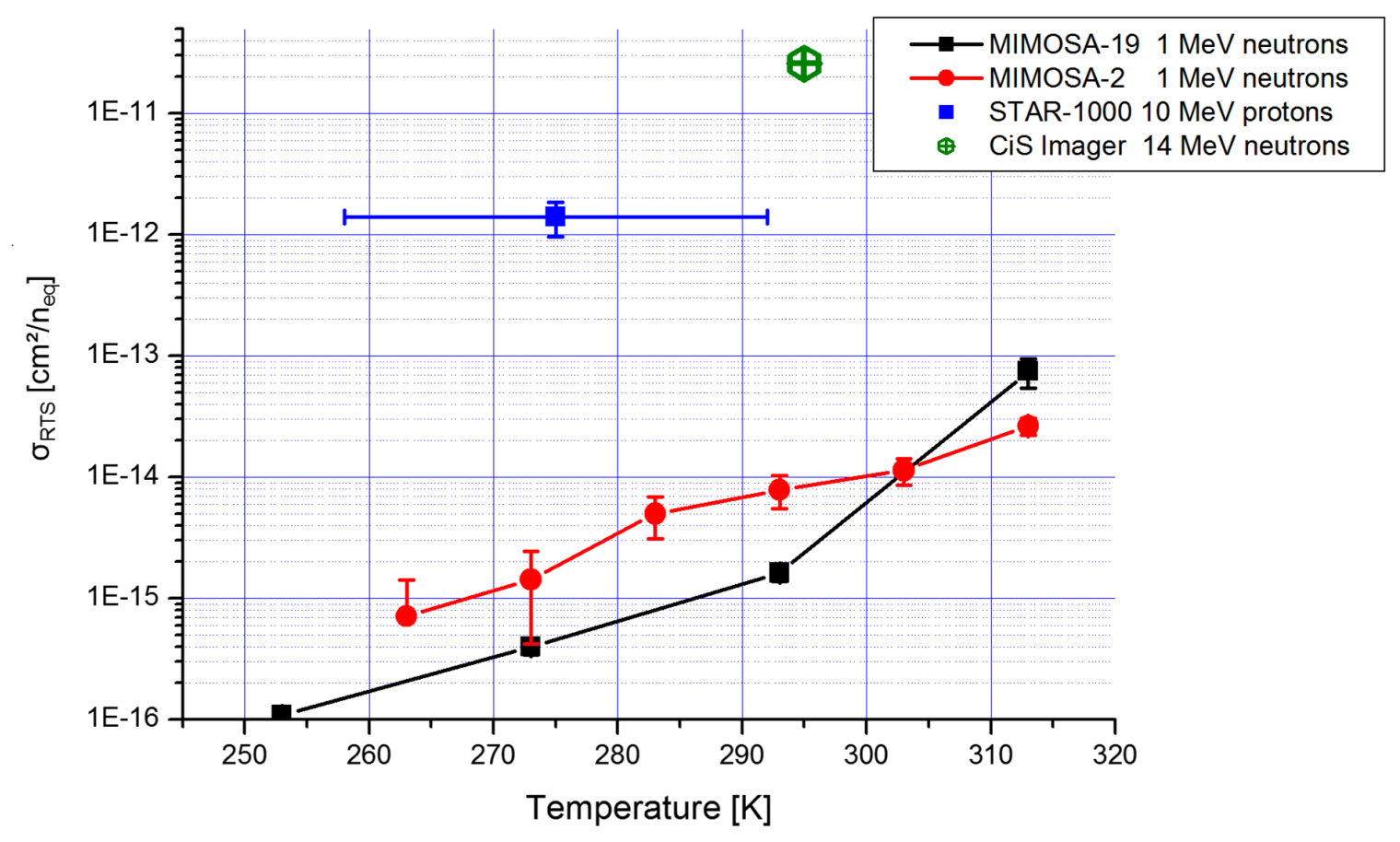

Figure 35: Cross-section for the creation of RTS-pixels as reported by different studies. Note that the reported data relies on different definitions of RTS. The approximate thresholds for the RTS amplitude are: $6.7 \mathrm{fA}$ for MIMOSA-19, [89], about $10 \mathrm{fA}$ for MIMOSA-2 [50], 1.25 . $10^{-16} \mathrm{~A}$ for STAR-1000 [84, about $10^{-17} \mathrm{~A}$, CiS Imager [85]. The cross-section is normalized to the surface of the pixel diodes.

one may define a cross-section for the generation of an RTS defect in the pixel like:

$$
N_{R T S}=\frac{A_{\text {diode }}}{A_{\text {pixel }}} \cdot N_{\text {pixel }} \cdot \sigma_{R T S}(T) \cdot \Phi_{\text {neq }}
$$

Here, $N_{R T S}$ denote the number of pixels showing RTS and $N_{\text {pixel }}$ represents the total number of pixels of the device under study. $\Phi_{n e q}$ stands for the radiation dose in units of $\mathrm{n}_{\mathrm{eq}} / \mathrm{cm}^{2}$ and $\sigma_{R T S}(T)$ for the temperature dependent RTS defect generation cross-section. $A_{\text {diode }}$ and $A_{\text {pixel }}$ represents the surface of the pixel and the pixel diode respectively. The relation of the surface of the pixel and the pixel diode was introduced in order to normalize the cross-section to the diode surface instead of the pixel surface. This follows the consideration that the non-depleted active volume of the pixel is likely not vulnerable to RTS. The cross-section for generating RTS-pixels may then be derived as:

$$
\sigma_{R T S}(T)=\frac{N_{R T S}}{N_{\text {pixel }}} \cdot \frac{A_{\text {pixel }}}{A_{\text {diode }}} \cdot \frac{1}{\Phi_{\text {neq }}}
$$

This cross-section has been computed for different studies on the creation probabilities of RTS. For tiny diodes, a $1 \mu \mathrm{m}$ ring was added in order to account somewhat for the side depletion of the diode. The results are shown in Fig. 35, which shows that the cross-section obtained depends substantially on the definition of an RTS pixel (see caption for details). 


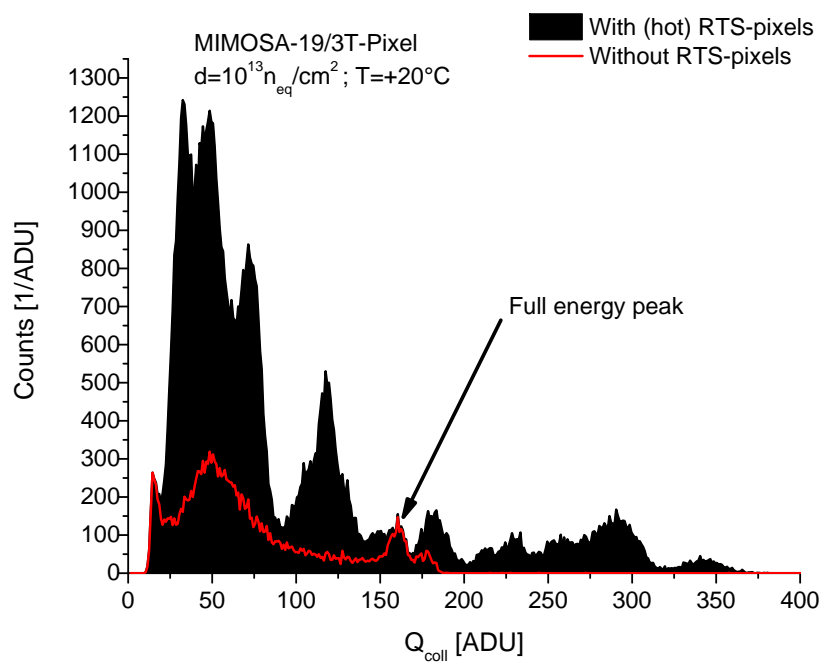

Figure 36: ${ }^{55} \mathrm{Fe}$ amplitude spectrum of a 3T-pixel sensor showing massive RTS after being irradiated with neutrons. Individual pixels generate long trains of hits with similar amplitude, which creates peaks in the spectrum. After masking those pixels, the X-ray amplitude spectrum becomes visible. From [82.

The results vary by multiple orders of magnitude, which is interpreted as dominated by the different effective detection thresholds $I_{\mathrm{N}_{\mathrm{RTS}}}$ used. An attempt to normalize the different results based on Eqn. 34 did not lead to a reasonable outcome. Instead, the data seems to scale very roughly with $\sigma_{R T S} \propto 1 / I_{\mathrm{N}_{\mathrm{RTS}}}$, which remains to be understood.

8.1.2.3 Impact of the diode RTS on 3T-pixels CPS indicate a particle hit, whenever the charge collection diode collects an excess charge exceeding the discrimination threshold within the integration time. The contribution of the diode leakage current to this charge is typically estimated by building a rolling average of the charge over past 100 frames and subtracted hereafter. This approach is suited to follow slow variations of the leakage current as caused by temperature fluctuations but fails to follow the abrupt changes caused by the RTS-fluctuations. In case the integrated amplitude of the RTS signal $Q_{R T S}=I_{R T S} \cdot t_{i n t}$ exceeds the discrimination threshold of the pixel, a hit is indicated and the update of the leakage current estimate is suspended. As RTS tends to keep its high state for a significant time, this may end up in a series of false hit indications, which only ends once the pixel fluctuates back to its lower state. All hits of this series will indicate the same signal charge and may thus generate peaks in the amplitude spectra, which have to be removed before analysing them (see Fig. 36).

More importantly, the RTS in the diodes of irradiated 3T-pixels dominate the dark hits indicated by the related sensors [83, 89]. A quantitative measurement of the related dark rate is shown in Fig. 37a. One observes that the related hit rate increases with the radiation dose and even more importantly with the temperature. The latter effect is considered as related to the temperature dependent increase of $I_{R T S}$ (see previous section). It is evident that, besides the use of cooling, accelerating the integration time of the sensor is a suited way to reduce the number of false hit indications caused by RTS. This is as this measure reduces $Q_{R T S}$. Moreover, it may be appropriate to mask some of the affected pixels.

8.1.2.4 Impact of diode RTS on SB-pixels SB-pixels are less vulnerable to RTS than 3T-pixels. This is because of the intrinsic leakage compensation, which follows the RTS 


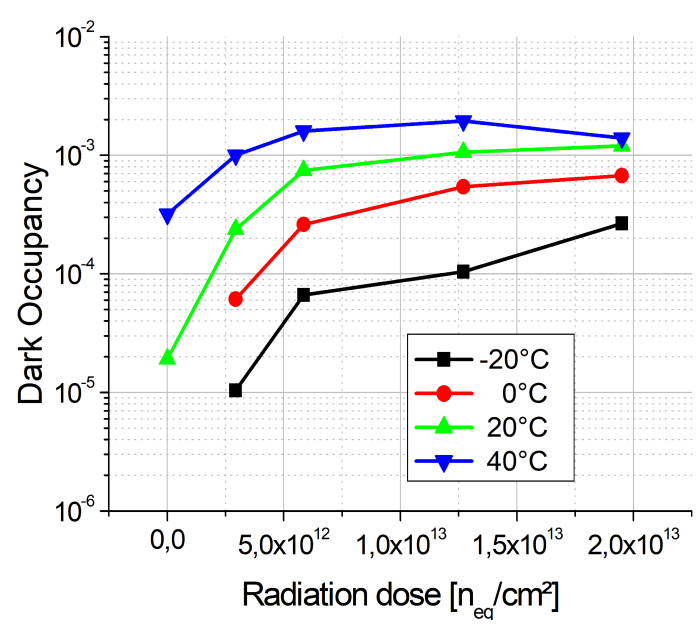

(a) Fake hit rates of the 3T-pixels of MIMOSA-19. Fake hits from both, RTSpixels and regular pixels are accounted for.

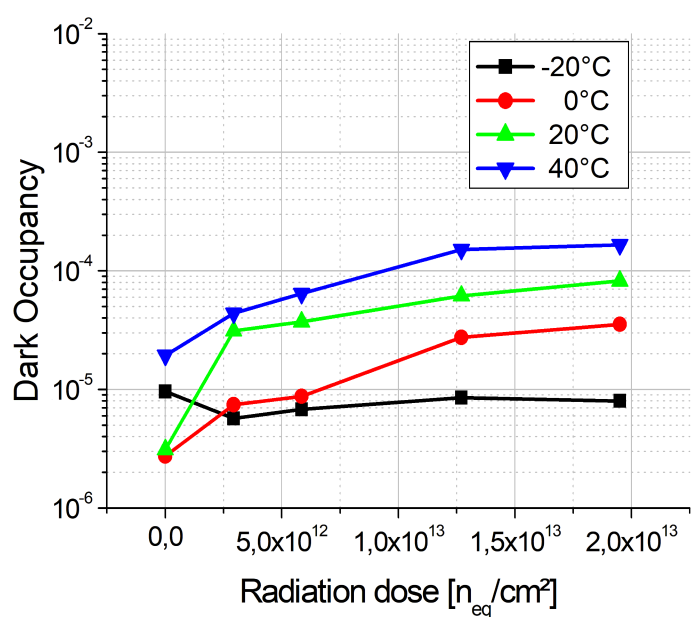

(b) Fake hit rates of the SB-pixels of MIMOSA-18. Fake hits from both, RTSpixels and regular pixels are accounted for.

Figure 37: Fake hit rates for CPS with SB- and 3T-pixels. Based on data previously published in [89]. See text.

induced steps of the leakage current within a reasonably short time. Fake hit indications are typically expected at the rising edge of the RTS signal while the falling edge may in theory generate a certain dead-time of the individual pixel. Consequently, one expects the individual RTS pixel to generate less fake hits if it has a SB-amplifier. The argument is somewhat weakened by the theoretical consideration that the biasing diode (despite of being forward biased) might also be affected by RTS, which would generate an additional source of noise.

The capability of SB-pixels for compensating the currents caused by RTS complicates identifying affected pixels. The signal charge indicated by the pixel does typically not show an RTS signature, no matter if it this signal is present or not. However, a pixel affected by RTS may be identified considering that the collection diode acts as switchable current source, which pulls a current through the biasing diode. Depending on the state of the current source, the voltage drop in the biasing diode changes, which may be detected by plotting the raw dark output signal of the pixel (witout RTS). Still, the measurement concept remains a rather qualitative tool. First of all, the voltage drop of the biasing diode shows a logarithmic response to the leakage current. Consequently, the voltage fluctuation caused by a certain $I_{R T S}$ decreases with the increasing static leakage current of the diode. Secondly, the detection concept is not really suited to distinguish between RTS occurring in the diode and RTS occurring in the input transistor of the pre-amplifier of the pixel (see next section).

In [89], the number of RTS-pixels was measured as function of the radiation dose and the temperature for a sensor relying on LR epitaxial layer and a standard SB-pixel. According to the indicated results of the measurements, most SB-pixels show occasional signatures of RTS from the start. The fraction of indicated RTS-pixels decreases with high temperatures and radiation doses. This suggests that the above mentioned detection issues apply as both parameters increase the static leakage current. For this reasons, the authors of the paper 
consider the measurement as non-reliable. As displayed in Fig. 37b, SB-pixels exhibit indeed a by about one order of magnitude lower dark hit rate than about similar 3T-pixels.

\subsubsection{RTS in MOSFET-transistors}

Besides in diodes, RTS can be found in FET transistors. Here, RTS modulates the sourcedrain current of the transistor. This RTS is among others caused by traps in the conductive channel nearby the gate of the transistors. The charges of those individual traps add to the gate voltage and thus modulate the electric current passing the device. The amplitude of the RTS depends on the precise position of the trap in the FET, which turns into a wide and continuous range of possible amplitudes. The superposition of multiple RTS sources is considered to cause the so-called $1 / \mathrm{f}$ - noise [90].

8.1.3.1 Properties of the RTS The typical current amplitude of the RTS signal is given with [91]:

$$
\frac{\Delta I_{D}}{I_{D}}=\eta \cdot \frac{g_{m}}{I_{D}} \cdot \frac{q}{W L C_{o x}} \cdot\left(1-\frac{x_{t}}{t_{o x}}\right)
$$

Here, $I_{D}$ is the drain current, $\Delta I_{D}$ the related RTS-amplitude, $\eta$ a process constant, $g_{m}$ the transconductance of the MOSFET, $q$ the elementary charge, $W$ and $L$ the gate dimensions, $C_{o x}$ the MOSFET gate oxide capacitance, $x_{t}$ the distance between the the trap and the $\mathrm{Si} / \mathrm{SiO}_{2}$ interface and $t_{o x}$ the gate thickness. From the equation, one concludes that the RTS amplitude increases with shrinking length and width of the transistor gate. This can be intuitively understood as a consequence of the limited range of the fields caused by the individual traps in the semiconductor. In [90] it is reported that this amplitude scales typically with $1 / L^{2}$, where $L$ denotes the length of the transistor gate. This is compatible with Equation 40 as $C_{\text {ox }}$ scales with $L$.

The amplitude of RTS is simulated in [92] for transistors with gates of less than $100 \times$ $100 \mathrm{~nm}^{2}$. It is reported that RTS becomes particularly important for transistors working in weak inversion at drain currents if $\lesssim 1 \mu \mathrm{A}$. The predicted RTS-amplitude may scale from $\sim 5 \%$ to $40 \%$ of the drain current for transistors with $100 \times 100 \mathrm{~nm}^{2}$ and $30 \times 30 \mathrm{~nm}^{2}$. Besides of the distance between the $\mathrm{Si} / \mathrm{SiO}_{2}$ interface and the trap, the location of the trap along length of the gate is a decisive parameter for the RTS amplitude. A maximum amplitude is reached by traps in the region of the minimum electron density in the middle of the gate.

8.1.3.2 Impact on CPS MOSFET RTS was reported to affect the input stage of the on-pixel pre-amplifier of CMOS pixels [93]. In the case of 3T-pixels and SB-pixels, this is the source follower transistor of the device. This transistor has to operate at low currents in order to limit the power consumption of the device. Moreover, a minimum gate size $(L \times W)$ is ambitioned in order to minimize the gate capacitx $C_{o x}$ and thus increase the charge-tovoltage conversion gain of the amplification stage. Simultaneously, a thin oxide thickness $t_{o x}$ is preferred to increase the tolerance of the transistor to ionizing radiation. All those design choices reduce the noise of a pixel, which is not affected by MOSFET RTS. However, accounting to Equation 40, they simultaneously worsen the properties of pixels, which are affected by this noise. 


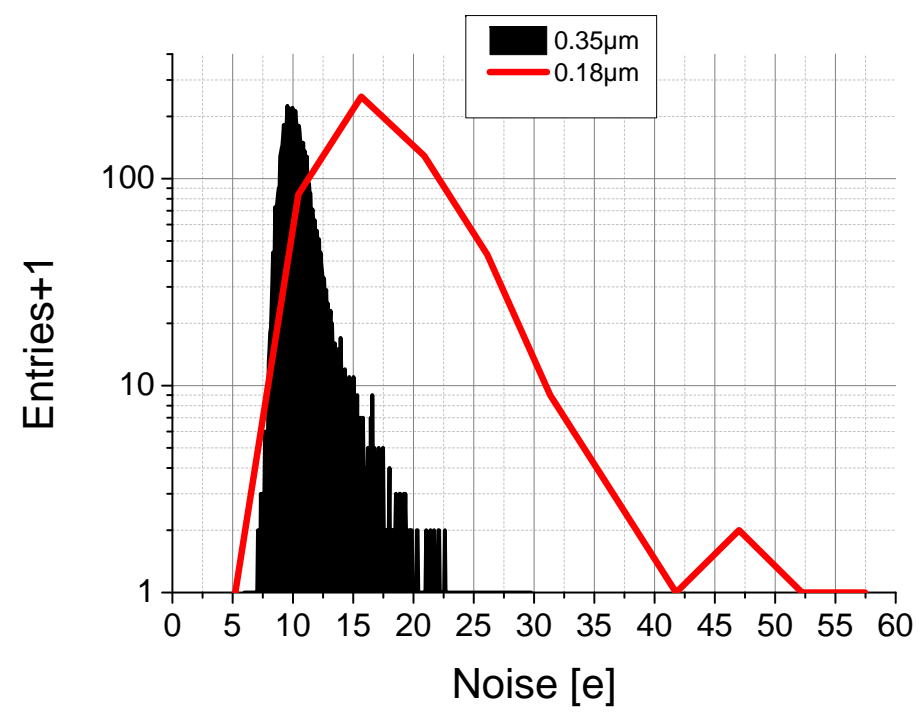

Figure 38: Noise distribution (noise vs. number of pixels showing this noise) of two CPS with SB-pixel engineered in a $0.35 \mu \mathrm{m}$ and in an $0.18 \mu \mathrm{m}$ CMOS process. Both imagers (MIMOSA18AHR and MIMOSA-32ter) were equipped with collection diodes with $11 \mu \mathrm{m}^{2}$ surface. The gate length of the SF-transistors was $0.35 \mu \mathrm{m}$ and $0.2 \mu \mathrm{m}$ respectively. From [52].

\begin{tabular}{|l|c|cc|ccc|c|}
\hline Matrix & & $\begin{array}{c}\text { Width } \\
{[\mu m]}\end{array}$ & $\begin{array}{c}\text { Length } \\
{[\mu m]}\end{array}$ & $\begin{array}{c}\text { Noise } \\
A D U\end{array}$ & $\begin{array}{c}\text { Gain } \\
{[e / A D U]}\end{array}$ & $\begin{array}{c}\text { Noise } \\
{[e]}\end{array}$ & $\begin{array}{c}\text { Noise } \\
99 \%<[e]\end{array}$ \\
\hline Mi-32ter-P2 & A & 1.5 & 0.2 & 1.81 & 11.1 & 19.8 & 41 \\
Mi-32ter-P6 & B & 0.9 & 0.2 & 1.97 & 10.5 & 20.5 & 55 \\
Mi-32ter-P5 & C & 0.5 & 0.2 & 2.09 & 10.1 & 21.3 & 63 \\
Mi-18AHR-A2 $(0.35 \mu \mathrm{m})$ & $\mathrm{R}$ & $\mathrm{N} / \mathrm{A}$ & 0.35 & - & - & 10.7 & 18 \\
\hline Difference A $\rightarrow \mathrm{C}$ & & & & $+15 \%$ & $+10 \%$ & $+8 \%$ & $+54 \%$ \\
\hline
\end{tabular}

Table 2: Noise of SB-pixels with $11 \mu \mathrm{m}^{2}$ diode surface manufactured in a $0.18 \mu \mathrm{m}$ CMOS process as a function of the SF-transistor gate size. Data on a pixel implemented in $0.35 \mu \mathrm{m}$ CMOS process is given for comparison. From [52]. See text.

The consequence of this behaviour is shown in Fig. 38. The figure shows a histogram of the pixel noise of two reasonably similar SB-pixel architectures, which were implemented in a $0.18 \mu \mathrm{m}$ and a $0.35 \mu \mathrm{m}$ CMOS process, respectively. The gate length of the SF transistors was essentially chosen to amount this minimum feature size, which turns for a smaller pixel capacitance of the "0.18 $\mu \mathrm{m}$-pixels". This lower capacitance improves the charge-to-voltage conversion gain of the pixel and thus was expected to amplify the signal while keeping the noise (in units of $\mathrm{mV}$ ) constant. Based on this consideration, one expects a drop in the noise (in units of $e \mathrm{ENC}$ ). This advantage can indeed be seen for some of the "0.18 $\mu \mathrm{m}$-pixels". However, most of those pixels exhibit a substantially higher noise than the "0.35 $\mu \mathrm{m}$-pixels".

Tab. 2 lists the results of a study on the impact of a modification of the width of the transistor gate of the SF-transistor in SB-pixels [94]. One observes that the average noise of the pixels increases slightly once the width of the transistor gate is reduced. This holds as the additional RTS found in the smaller transistor dominates the small benefit in pixel capacitance, which remains modest due to the dominance of the diode capacitance. Consequently, the pixels with biggest gate show the lowest average noise. The effect is not particularly pronounced for the average noise, it becomes sizeable for the exhibiting highest pixel noise. 
While the noise of $99 \%$ of all pixels remains below $41 e \mathrm{ENC}$ for the pixel with the largest gate, this value increases to $63 e$ ENC for the smallest gate. As the pixels of a CPS have typically a common threshold setting, which is guided by the most noisy pixels, an optimization of the pixels for MOSFET RTS seems of advantage even if the average pixel noise increases slightly.

Additional valuable data on the impact of the length of the transistor gate on the noise (in units of $\mathrm{mV}$ ) is provided in [95]. The results suggest that for $W=1.5 \mu \mathrm{m}$, the average noise increases with increasing transistor length while the width of the noise distribution decreases. A good compromise between both parameters is given with $L=0.8 \mu \mathrm{m}$ to $L=1.0 \mu \mathrm{m}$.

The outcome of the different studies suggest that the noise of CPS produced with CMOS processes with a feature size of $0.35 \mu \mathrm{m}$ and above is dominated by the pixel capacitance. For CMOS processes with a feature size of $0.18 \mu \mathrm{m}$ and below, the RTS in the input transistor of the amplifier dominates. This holds unless the size of the gate of this transistor is intentionally increased. Indeed, this strategy was found to reduce the noise of pixel produced in a $0.18 \mu \mathrm{m}$ CMOS process [96].

8.1.3.3 Impact of radiation damage on RTS in MOSFETs Few is known on the impact of radiation damage on the RTS noise of MOSFETs. A pioneering study carried out with a n-channel poly-SI gate MOSFET and an effective gate size of $L=1.2 \mu \mathrm{m} / \mathrm{W}=0.5 \mu \mathrm{m}$ is presented in [97. The study reports an appearance of multiple RTS states after applying an X-ray dose of up to $\sim 1$ Mrad. Simultaneously, the disappearance of some RTS states existing prior to the irradiation was observed. Most or the radiation effects could be recovered after annealing the device at $T=400^{\circ} \mathrm{C}$.

A first study on a commercial CPS manufactured in a $0.18 \mu \mathrm{m}$ CMOS process was done by exposing the device to up to $2.19 \mathrm{Mrad} \mathrm{X}$-rays [95]. The sensor was operated with a integration time of $2 \mu \mathrm{s}$ and a mean noise of $194 \mu \mathrm{V}$ is reported. About $0.4 \%$ of the 3Tpixels of the sensor exhibited a significant MOSFET-RTS prior to irradiation. This number was increased starting from a dose of few $100 \mathrm{krad}$ and by up to $50 \%$ after the highest dose applied. As for the previous study, modifications of the RTS amplitude and frequency are reported.

\subsection{Effects of thermal and cold neutrons}

As discussed in Sec. 2.2, the NIEL-model neglects by construction effects of the device chemistry and possible reactions of trace elements found in the irradiated silicon. This was suspected to generate a bias in the radiation tolerance studies of CPS, as their p-doped epitaxial layer is typically doped with boron. Being exposed to cold and thermal neutrons, the dopants show a huge cross-section for the neutron induced fission $\left(\mathrm{n}+{ }^{10} \mathrm{~B} \rightarrow{ }^{7} \mathrm{Li}+{ }^{4} \mathrm{He}+2.8 \mathrm{MeV}\right)$ and thus add a radiation energy, which is ignored by the NIEL-model.

First studies addressing the question on whether this fission may generate significant radiation damage were carried out with with n-p-n transistors realized in the DMILL process [98, 99]. The transistors were irradiated with fast and thermal neutrons and the radiation damage was compared. It was observed that thermal neutrons damage the transistors stronger than $1 \mathrm{MeV}$ neutrons, which is in strict contradiction to the standard NIEL tables. 


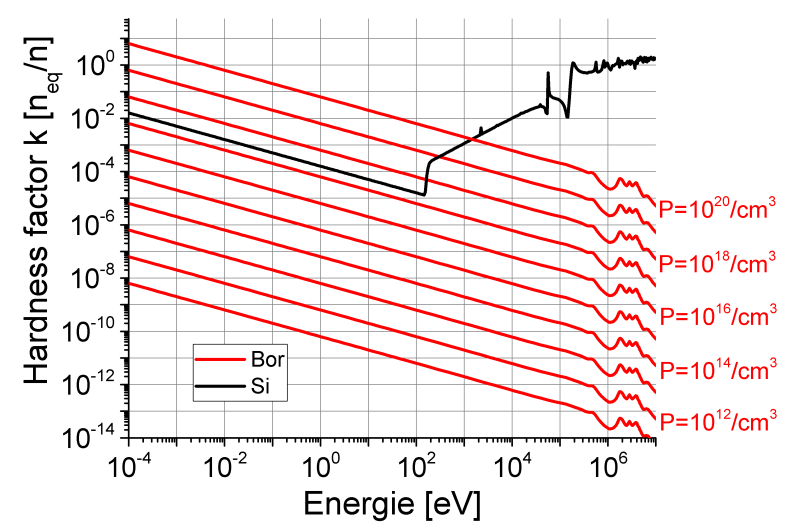

Figure 39: Estimated hardness factor of neutrons impinging pure silicon (black, data from [28]), predicted damage according due to boron fission (red). Warning: Model predictions do not match experimental data, see text. From [100].

The question, if this effect may generate additional radiation damage in CPS, was addressed in a theoretical and experimental pilot study [100]. The NIEL caused by the fission process was estimated for natural boron based on the energy dependent cross-section of the neutron capture process causing it. The fission energy of $2.8 \mathrm{MeV}$ was distributed by means of energy and mass conservation to the ions. The frequent generation of a $\gamma$-ray in the process was neglected. The movement of the ions in the silicon and the bulk damaged was simulated with the dedicated software SRIM [101]. The hardness factor of the ions was estimated by comparing the number of vacancies indicated by this software with the one of protons simulated with the same device. The results of the study are displayed in Fig. 39. They suggest that the effect of boron fission may provide a significant contribution to the over-all NIEL starting from doping concentrations above $P=10^{17} / \mathrm{cm}^{3}$.

This value is not reached in the sensitive volumes of CPS but e.g. in the substrate of the sensors and in the P-Wells / P-Diffs used for implementing transistors. Therefore, one does not expect the sensitive volume of CPS to take particular damage in first order. However, according to the simulation, the fission products show a certain range $\left(6 \mu \mathrm{m}\right.$ for ${ }^{4} \mathrm{He}$ and $3 \mu \mathrm{m}$ for ${ }^{7} \mathrm{Li}$ ). Therefore, ions created in the highly doped structures may create indirect damage in the more moderately doped structures.

The experimental part of the study was carried out with MIMOSA-19 sensors, which rely on a $14 \mu \mathrm{m}$ thick, LR epitaxial layer with an anticipated P-doping of few $10^{15} / \mathrm{cm}^{3}$. The $196 \times 1963$ T-pixels with $12 \times 12 \mu \mathrm{m}^{2}$ pitch have L-shaped diodes with $39.6 \mu^{2}$ surface. The anticipated doping of the substrate of the P-Well is $\sim 10^{19} / \mathrm{cm}^{3}$. The chips were irradiated at the FRM II research reactor in Garching. The MEDAPP beam line 102 provided fast neutrons (a direct fission spectrum with $E_{\text {neutron }}=2 \mathrm{MeV}$ (peak) and a hardness factor $k \approx 11 \mathrm{MeV}$ neutron equivalent per neutron, $E_{\text {neutron }}>100 \mathrm{keV}$ for $99 \%$ of all neutrons). Unwanted $\gamma$-rays caused an ionizing dose of $<100 \mathrm{krad}$ per $10^{13} \mathrm{n} / \mathrm{cm}^{2}$. Other sensors were irradiated at the PGAA beam line [103] with cold neutrons $\left(E_{\text {neutron }}=1.8 \times 10^{-3} \mathrm{eV}\right.$ (peak), $k \approx 0.003 \mathrm{~N}_{\text {eq }}$ ) and an unknown ionizing dose. The irradiation was done at room temperature. Despite the sensors remained unpowered during irradiation, the ionizing radiation damage is 


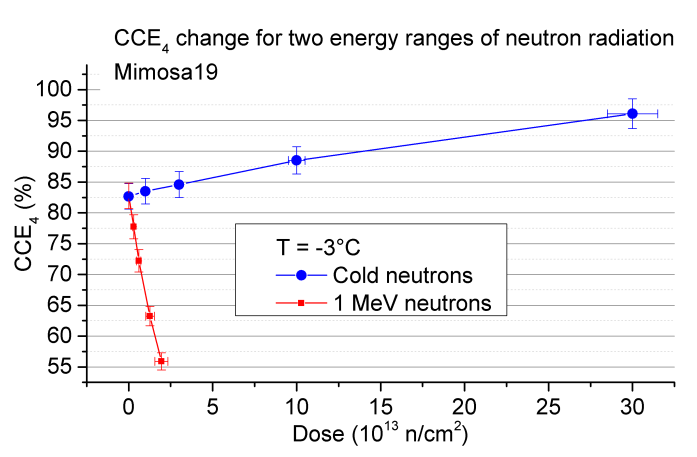

(a) The charge collection efficiency of MIMOSA-19 for the four most significant pixel in a $5 \times 5$ cluster as a function of the neutron dose and neutron energy. From [100].

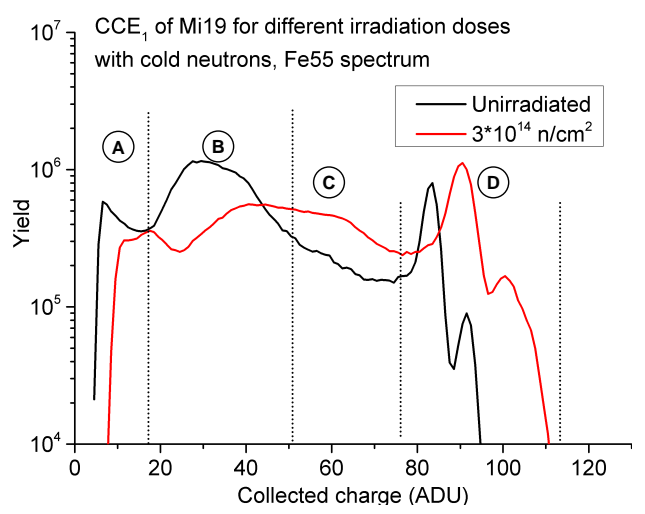

(c) Amplitude spectrum of a reference sensor and a sensor irradiated with cold neutrons. From [100.

Figure 40: Charge collection and ${ }^{55}$-Fe spectra of sensors exposed to cold $(1.8 \mathrm{meV})$ and fast $(\sim 1 \mathrm{MeV})$ neutrons. Mind the scale while comparing the spectra.

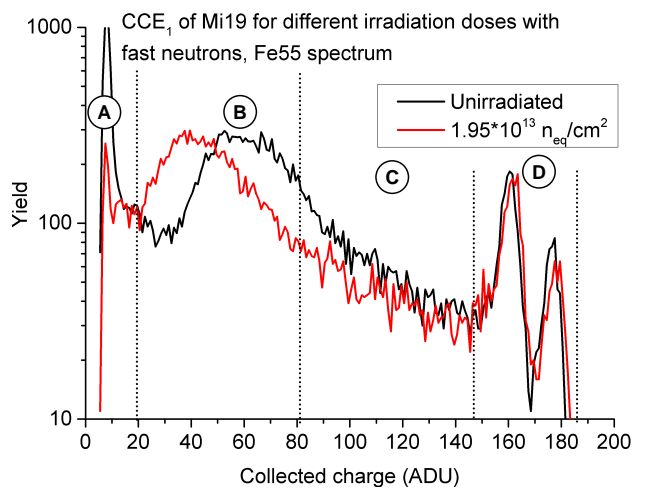

(b) Amplitude spectrum of a non-irradiated MIMOSA-19 and of a MIMOSA-19 sensor after irradiation with $1 \mathrm{MeV}$ neutrons. From [100]. 
considered to determine the leakage currents after irradiation.

The sensors were bonded, illuminated with X-ray sources $\left({ }^{55} \mathrm{Fe} \rightarrow 5.9 \mathrm{keV},{ }^{109} \mathrm{Cd} \rightarrow 22.1 \mathrm{keV}\right)$ and their amplitude spectra were recorded. The results of the study are displayed in Fig. 40a. As expected, the sensors irradiated at MEDAPP display a decrease of the average CCE with increasing radiation dose. Unexpectedly, the sensors irradiated at PGAA show an increase of the CCE. The slope of the CCE does thus depend on the neutron energy, what cannot be explained with a trivial quantitative modification of the NIEL scaling and thus also contradicts the theoretical results displayed in Fig. 39.

An analysis of the related ${ }^{55} \mathrm{Fe}$ amplitude spectra suggest an explanation for this finding. As illustrated in Fig. 40b, the sensors exposed to the fast neutrons show the usual response to the radiation load (compare Sec. 6.1.1), which is a reduced charge collection efficiency and an unchanged calibration peak. The latter suggests that the properties of the photo-diode including the doping of the epitaxial layer was not changed. The response of the sensor, which was irradiated with cold neutrons, is qualitatively different. As displayed in Fig. 40c, the calibration peak of the amplitude spectrum increases in size after irradiation. Moreover, the position of the peak is displaced. Moreover, one observes an improved CCE in the epitaxial layer, which manifests itself by a displacement of the big charge collection peak toward higher values. A comparison of this signature with the findings on sensors with depleted active volume (see Sec. 6.4.3) suggests that this signature corresponds to an increase of the depleted volume of the diode. As the voltage applied to the charge collection diode remained unchanged, this increase would plausibly be created by a reduced doping of the active volume of the sensor.

The findings can be explained by a strong, radiation induced acceptor removal, which reduces the doping of the epitaxial layer and such increases the size of the depleted zone of the diode. The numbers of entries in the calibration peaks increases by a factor slightly above two. For flat PN-junctions, this would turn into a drop in doping by a rough factor of five but higher values should be considered for the small charge collection diodes of CPS. Such a acceptor removal is also known from different studies (see Sec. 2.2). However, one would have to assume a hardness factor of about unity for the cold neutrons, which fits the findings on the DMILL transistors reported above but is two to three orders of magnitude above the predictions of the NIEL-model.

Despite the details remain to be understood, it is safe to state that the damage caused by thermal neutrons in CPS is not predicted correctly by the NIEL-model. Dedicated tests are thus recommended before operating CPS in a harsh radiation field caused by thermal neutrons.

\subsection{Radiation damage caused by relativistic heavy ions}

Vertex detectors are typically installed very close to the beam axis. Therefore, they are often exposed to primary beam particles from the so-called beam halo. Moreover, they may suffer from impacts of the full beam during beam tuning or in case the steering system fails. This is particularly worrying in heavy ion experiments as each beam ion has the potential to created irreversible radiation damage. Obvious methods for protecting the sensors consist in collimating the beam, adding an emergency stop system detecting beam displacements to the 
accelerator and installing the vertex detector on a moving table. The latter allows to move the detector out during beam tuning but it is typically too slow to protect the device in case of an unwanted beam displacement.

According to the Bethe-Bloch-equation, the energy deposit caused by a beam ion with a charge $Z$ scales with $Z^{2}$. Consequently, the energy deposit of a $\mathrm{Pb}$-ion in silicon exceeds the one of a proton by close to four orders of magnitude. Depending on the structure of the beam halo, the total ionizing and non-ionizing doses induced by the beam ions may provide even a dominant contribution to the total radiation exposure of the sensors. Moreover, due to the high charge deposit, even individual ions may generate damaging effects in a CMOS device. Those are referred to as single event upsets (SEU). Relevant SEUs in CPS are changes of the logic state of a memory cell (bit flip) and the generation of a meta-stable short circuit in the device (latch-up). While the bit flip may be tolerated in most cases, latch-ups may destroy CPS irreversibly and call for protection measures.

\subsubsection{Single event upsets and latch-up}

SEUs occur, if a particle deposits a sufficient amount of charge at a sensitive point of a CMOS device. The sensitive surface is typically small as compared to the total surface of the device and the charge deposit is subject to a Landau-fluctuation. The probability of obtaining an SEU is typically expressed as a cross-section, which depends on the nature and energy of the impinging particle.

The cross-section for the occurrence of single bit flips caused by ionizing particles was studied during the preparation of the the ALICE Inner Tracking System [96]. Various memory cells manufactured in $0.18 \mu \mathrm{m}$ CMOS technology were exposed to slow (25-250 MeV) protons, which show a charge deposit similar to the one of relativistic heavy ions. The cross-section for bit flips was found to amount in the order of $10^{-13} \mathrm{~cm}^{2} /$ bit. This value is not considered as particularly worrying for data memories as individual bit errors in the data stream of the sensors can typically be tolerated. More care is needed for the registers storing the information required for the internal control of the sensors. A bit flip in those registers may disturb the sensor operation, which has to be detected and to be cured by restarting the device. In order to reduce the down-time of the sensors, the ALICE collaboration decided to protect those registers. This can for example be done by storing each bit in three memory cells and by deciding about the correct state by majority voting. A bit flip in individual cells can then be tolerated and cured on the fly.

Latch-ups occur in vulnerable CMOS structures as for example in the CMOS inverter displayed in Fig. 41a This inverter is composed from a PMOS and an NMOS field effect transistor, which are realized by $\mathrm{N}+$ implantations and an $\mathrm{N}$-well with $\mathrm{P}+$ implantations respectively. As shown in the figure, this CMOS structure realizes unintentionally a combination of two bipolar transistors, which are switched of in the normal state of operation. This may change in case a major current is generated in the reverse biased $\mathrm{P}$-epi/N-well junction. In this case, the resistivity of the silicon material (represented by $R_{P}$ and $R_{N}$ in the figure) generates a voltage drop. This voltage drop may be sufficient to switch one of the parasitic transistors into a conductive state. In this case, the voltage drop at the opposite resistor is increased and the second bipolar transistor becomes conductive, which increases the initial 


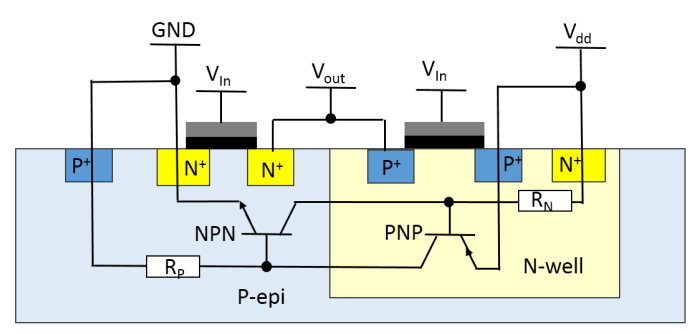

(a) CMOS inverter and bipolar transistors making the structure vulnerable for latch-up. After an illustration by "Cepheiden" found on Wikipedia.

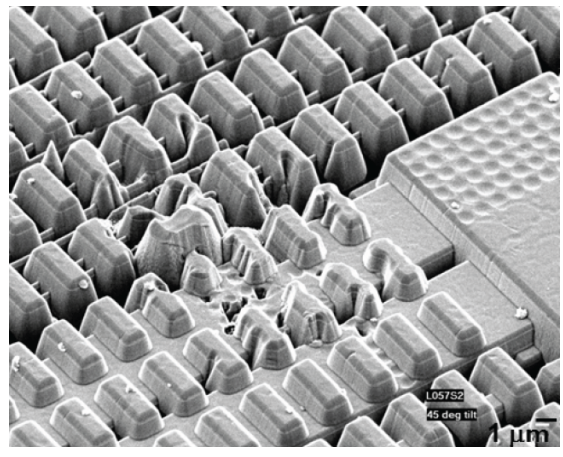

(b) Metal layer of the damaged region of an ULTIMATE sensor failing permanently after a latch-up event. Some metal structures seem to have melted. From 104.

Figure 41: Latch-up, mechanism and damage.

current further. Finally, both bipolar transistors are stabilized in the conductive state. Therefore, rather large currents may pass between $V_{d d}$ and GND and the ohmic losses at $R_{P}$ and $\mathrm{R}_{\mathrm{N}}$ may heat up the structure until it is thermally destroyed. The conductive state may be switched back to the initial, non-conductive state by switching off the power of the device and restarting it. If this action is undertaken with sufficient speed, no damage is to be expected.

The probability of latch-up was studied [61] with the MIMOSTAR-2 sensor (a sensor relying on SB-pixels) in the prospective of using the device for the PXL detector of STAR. No latch-up was observed for LET values up to $8 \mathrm{MeV} \cdot \mathrm{cm}^{2} / \mathrm{g}$. Hereafter, a sharp increase of the related cross section was observed and a cross section of roughly $5 \times 10^{-4} \mathrm{~cm}^{2}$ is reported for a LET above $30 \mathrm{MeV} \cdot \mathrm{cm}^{2} / \mathrm{g}$. According to the study, also soft upsets were observed. The related cross section was $2 \times 10^{-7}$ to $7 \times 10^{-7} \mathrm{~cm}^{2}$ at the highest LET applied $\left(13 \mathrm{MeV} \cdot \mathrm{cm}^{2} / \mathrm{g}\right)$, which is a small factor above the related latch-up cross section.

Latch-up was found to be the major source of losses of sensors in the STAR-PXL detector, which was carried out with ULTIMATE CMOS sensors [48, 105]. The sensors were protected against damage caused by latch-up by a dedicated power supply, which was measuring the current consumed by a ladder of 10 sensors and by performing a power cycle, if this value exceeded a certain threshold value. However, in a first run carried out in 2014, $14 \%$ of the active surface of the detector was lost during the first two weeks of operation [104]. The damage was concentrated to the most inner layer of the detector. After reducing the activation threshold of the latch-up protection system from initially $400 \mathrm{~mA}$ to $120 \mathrm{~mA}$ above the nominal current, only four additional sensors were lost during the 10 remaining weeks of operations. Operating the sensors with an over-current protection threshold of $80 \mathrm{~mA}$ above the nominal current consumption reduced the number of lost sensors to 5 during each, the RHIC run in 2015 and 2016.

A follow-up study was carried out by bombarding individual sensors and ladders with protons and heavy ions produced by the $88^{\prime \prime}$ Cyclotron BASE Facility at LBNL. Current limited latch-up states with a typical increase of the digital current of $300 \mathrm{~mA}$ were observed. A permanent damage could only be reproduced in sensors, which were previously thinned to 
$50 \mu \mathrm{m}$. The $700 \mu \mathrm{m}$ thick sensors studied were found to be reasonably robust. The location of the damage structures was identified through infrared camera inspection. The substrate and the epitaxial layer of the damaged sensors were removed in the region of interest. Electron microscope pictures of the damaged region revealed that the metal lines in the damaged regions were melted down (see Fig. 41b).

\subsubsection{Integrated displacement damage caused by heavy ions}

While the total ionizing dose caused by relativistic heavy ions in silicon can be estimated based on the Bethe-Bloch-equation, few is known concerning the integrated NIEL caused by those particles. Computations on the NIEL caused by up to $1 \mathrm{GeV}$ (not $\mathrm{AGeV}$ ) heavy ions impinging silicon may be found in [106] suggest that the radiation damage caused by slow gold ions may exceed $\sim 10^{6} \mathrm{n}_{\text {eq }} / \mathrm{cm}^{2}$. The numbers are in reasonable agreement with the experimental results reported in [107]. Only recently, a careful experimental study on the damage caused by $40.5 \mathrm{AMeV}{ }^{40} \mathrm{Ar}$-ions relying on depleted $\mathrm{p}^{+}-\mathrm{n}-\mathrm{n}^{+}$silicon detectors [108] was published. The results of this study suggests that those ions generate a non-ionizing radiation damage equivalent to $\sim 10 \mathrm{n}_{\mathrm{eq}} / \mathrm{cm}^{2}$. As stated by the authors, this result is not compatible with the previously mentioned theoretical calculations and observations, which remains to be understood. In any case, the studies do in any case not cover the ion energy of primary beam particles of relativistic heavy ion physics.

An estimate for the NIEL caused by relativistic ions is provided in [109]. In accordance with the focus of the work, the natural ion spectrum of cosmic radiation, this study limits itself to energies of $2 \mathrm{AGeV}$ to atoms up to $Z=26(\mathrm{Fe})$. The authors underline that nuclear fragmentation is to be considered at high ion energies. For light ions, namely for protons, the fragmentation of the target atom provides a significant contribution to the overall NIEL. For atoms with high atomic numbers $Z$, the NIEL scales approximately with $Z^{2}$. For $\gg 1 \mathrm{AGeV}$ energy range of interest for heavy ion physics, the NIEL seems to decrease with increasing particle energy, which would be consistent with the NIEL tables for protons. A naive extrapolation of the values toward higher $Z$ values suggests, that the NIEL of a Pb-ion with an energy above $2 \mathrm{AGeV}$ should be below few $100 \mathrm{n}_{\text {eq }} / \mathrm{cm}^{2}$.

The validity of this rough assumption was cross checked with an experiment [13]. Four CPS (MIMOSA-34) were exposed to primary $30 \mathrm{AGeV} \mathrm{Pb-ions} \mathrm{at} \mathrm{the} \mathrm{CERN-SPS.} \mathrm{Those} \mathrm{sensors}$ were developed by the IPHC Strasbourg and manufactured in a TOWER/JAZZ $0.18 \mu \mathrm{m}$ CMOS process with $18 \mu \mathrm{m}$ high resistivity epitaxial layer. They host SB-pixel with various pixel pitches. The tolerance of those pixels to $\sim 1 \mathrm{MeV}$ neutrons was measured independently, which allowed to compare the radiation damage caused by the heavy ions with the one caused by the neutrons.

The sensors were intentionally not powered but wrapped into aluminium foil and mounted on a holding structure made from plastic material. They were placed $200 \mathrm{~m}$ upstream the target in the beam line of NA61/SHINE. Dosimetry was provided by an ion sensitive, $4 \times 4 \mathrm{~mm}^{2}$ small scintillator. This scintillator together with its light guide and a suited, small PMT were placed on the support upstream the sensors. The signals of the PMT were forwarded to the NA61/SHINE counting house and the dosimetry was performed by means of single ion counting. During two weeks of operation, a maximum ion flux of $1.2 \times 10^{10} \mathrm{~Pb}$ ions $/ \mathrm{cm}^{2}$ 
was integrated. Hereafter, the sensors were bonded at the IPHC Strasbourg and illuminated with X-rays from a ${ }^{55} \mathrm{Fe}$-source. No significant drop of CCE was observed, which suggests that the equivalent radiation damage caused by the ions was $\lesssim 3 \times 10^{12} \mathrm{n}_{\mathrm{eq}} / \mathrm{cm}^{2}$. The noise of the pixel was also checked and again no significant noise increase due to irradiation was observed. This preliminary finding provides an upper limit for the hardness factor of $30 \mathrm{AGeV}$ $\mathrm{Pb}$ ions, which is given with $k_{i o n} \lesssim 300 \mathrm{n}_{\text {eq }}$. This upper limit is compatible with the above mentioned theoretical predictions. From the practical point of view, it allows to conclude that the integrated non-ionizing radiation damage caused by relativistic heavy ions in CPS will typically be dominated by the related ionizing damage. 


\section{Summary and conclusion}

CPS form an emerging technology for charged particle tracking. Their advantage with respect to other silicon pixel detector technologies consist in an excellent spatial resolution, a light material budget, a reliable particle detection and a low price. Their rate capability was initially moderate but improved dramatically during the last decade.

The tolerance of CPS to radiation was subject to intense research. For sensors relying on classical, partially depleted active volumes, the tolerance of the sensors to non-ionizing radiation is limited mostly by two radiation effects. Those are the radiation induced reduction of the lifetime of the minority charge carriers in the silicon, which tends to absorb signal electrons before they are collected; and the radiation induced increase of leakage currents. The latter causes an increase of shot noise and, in the so-called SB-pixels, an accelerated clearing of the pixel signal in the pre-amplifier.

The reduction of the lifetime of the signal electrons can be compensated by accelerating the collection of the signal charge. This collection was initially carried out by means of slow thermal diffusion, which created a dedicated vulnerability of the technology. A strong increase of the radiation tolerance with decreasing pixel size is observed, most plausibly because smaller pixels come with shorter average diffusion paths. Moreover, one may deplete the active volume of the sensor and thus collect the electrons by means of electric fields. A partial depletion became feasible once CMOS industry provided wafers with reduced doping in the epitaxial layer. This technological progress turned into an improvement of one order of magnitude in radiation tolerance. However, no full depletion was reached as the voltage applied to CMOS devices is restricted, which limits the depletion voltage. This limitation was bypassed only recently. The novel option of building fully depleted sensors allowed to accelerate the charge collection substantially. Fully depleted CPS prototypes with small size diodes as presented in this work were found to tolerate $5 \times 10^{14} \mathrm{n}_{\mathrm{eq}} / \mathrm{cm}^{2}$ without showing a crucial drop in charge collection efficiency, which was the highest radiation dose applied. This represents an improvement of three orders of magnitude with respect to the initial performance of the technology and solves the primary weak point of the technology. Even higher radiation tolerances were recently reported for sensors relying on large fill factor diodes or modified CMOS processes (see e.g. [110, 111]), which are however not subject of this work.

As ways to overcome the vulnerability of the charge collection process of CPS to nonionizing radiation damage are being found, secondary issues gain importance. This is in particular the radiation induced increase of leakage currents. The consequences of this increase must be controlled by means of cooling and accelerating the readout/shaping time of the pixels. While the prior solution is limited by practical constraints, the acceleration of the readout is in phase with the design goals of future experiments.

The primary effect of ionizing radiation consists in creating leakage currents, namely in the collection diode of the device. The reset transistor of 3T pixels may create an additional and dominating leakage current. Again, those leakage currents may endanger the safe operation of the device due to increased shot noise and accelerated signal clearing, which may be counteracted by means of fast readout and cooling. Moreover, thermal annealing may reduce this radiation damage significantly. The currents may be reduced by design by adding 
dedicated guard rings to the diodes. Those push vulnerable, thick $\mathrm{SiO}_{2}$ away from the diode and cut conductive paths. The use of CMOS-processes with small feature size is of use for increasing the radiation tolerance of transistors but does not necessarily help to increase the radiation tolerance of the diodes. This is as the thinner and thus more radiation hard gate oxide provided by those processes does not improve the properties of diodes, which are surrounded by other, thick oxide structures. The tolerance of CPS to ionizing radiation depends significantly on the specific design of the device. For imagers relying on simple pre-amplifiers, a tolerance to $\gtrsim 10 \mathrm{Mrad}$ was observed [94. Highly integrated sensors relying on clamping pixels were found to withstand 1.6 Mrad. Both were the highest dose applied.

Both, ionizing and non-ionizing are found to create Random Telegraph Signal in the charge collection diodes of CPS. This noise manifests itself as a fluctuation of the dark signal of the pixel between two or more well-defined levels. It is suited to generate a substantial number of false hit indications and to transform a pixel into a hot pixel. While the definition on whether a pixel shows RTS is not precise in literature, it is appropriate to state that all pixels show this feature after moderately high radiation doses. At the time given, no strategy to reduce RTS by design is known. However, the dark occupancy caused by this effect may be reduced by means of cooling the sensors and accelerating the readout. Moreover, SB-pixels show by design a substantially lower RTS induced dark rate than 3T-pixels. RTS is also observed in transistors and may generate false hit signatures in case the input stage of the pixel preamplifiers is affected. Increasing the surface of the related transistor gate is a known and suited approach for reducing the amplitude of the RTS signal below the detector threshold. The additional pixel capacitance generated by this step is found to increase the average noise of the pixels but to improve the overall performance of the device.

CPS are formed from p-doped silicon. Cold and thermal neutrons may induce a nuclear reaction of the dopants $\left(\mathrm{n}+{ }^{10} \mathrm{~B} \rightarrow{ }^{7} \mathrm{Li}+{ }^{4} \mathrm{He}+2.8 \mathrm{MeV}\right)$. The decay products may generate a substantial NIEL, which is not accounted for in the standard NIEL model. According to theoretical estimate within the framework of the NIEL model, this contribution dominates as soon as the doping concentration exceeds a value of few $p=10^{17} / \mathrm{cm}^{3}$. Tests performed with sensors, which were irradiated with cold $(1.8 \mathrm{meV})$ neutrons, showed a qualitatively different picture. It is observed that the neutron radiation generates an unexpectedly strong acceptor removal, which is about equal to the one expected for fast $1 \mathrm{MeV}$ neutrons and thus exceeds the predictions of the standard NIEL model by three orders of magnitude. In the LR-sensors tested, this beneficial effect dominated the expected recombination of signal charge. Therefore, the overall charge collection properties of the device was improved.

In heavy-ion experiments, CPS are exposed to impacts of direct heavy ions. This may generate a so-called latch-up, which is reversible short circuit in the devices. Unless this short circuit is extinguished by means of a rapid power cycle, it may generate a permanent thermal destruction of the sensor. This effect was observed to cause a substantial loss of sensors in the HFT of the STAR experiment until the activation threshold of the automatic power cycle was reduced. Post studies revealed that $50 \mu \mathrm{m}$ thin sensors are substantially more vulnerable to this effect than the standard $700 \mu \mathrm{m}$ sensors, which were previously used by STAR to study this effect. Besides this single event effect, the integrated NIEL of ions may damage the sensors and the related hardness factor was initially unknown. A related study suggests 
that a fully stripped $30 \mathrm{AGeV} \mathrm{Pb-ions} \mathrm{creates} \mathrm{an} \mathrm{equivalent} \mathrm{damage} \mathrm{of} \lesssim 300 \mathrm{n}_{\text {eq }} / \mathrm{cm}^{2}$.

Over all, the performances of CPS could be substantially improved during the last two decades. This includes an improvement of their radiation tolerance, which amounts two to three orders of magnitude. Thanks to those progresses and to the novel design options provided by next generation CMOS processes, the technology is now ready for a by far extended number of applications in detectors of heavy ion and particle physics. 


\section{References}

[1] Physics with Integrated Cmos Sensors and ELectron machines (PICSEL) group of the Institut Pluridisciplinaire Hubert Curien (IPHC) Strasbourg. Homepage and team members: http://www.iphc.cnrs.fr/-PICSEL-.html

[2] R. Turchetta et al., A monolithic active pixel sensor for charged particle tracking and imaging using standard VLSI CMOS technology, NIM-A 458 (2001) 667-689

[3] G. Deptuch et al., Development of monolithic active pixel sensors for charged particle tracking, NIM-A 511 (2003) 240-249

[4] D. Contarato et al., Beam-test of CMOS pixel sensors with 6 GeV electrons, NIM-A 565 (2006) 119-125

[5] F. Richard, J.R. Schneider, D. Trines, A. Wagner (editors), TESLA Technical Design Report Part I: Executive Summary, ISBN 3-935702-00-0, arXiv:hep-ph/0106314v1

[6] T.Behnke, S.Bertolucci, R.D. Heuer, R. Settles (editors), TESLA Technical Design Report, PART IV, A Detector for TESLA, Available at: http://tesla.desy.de/new_pages/TDR_CD/start.html

[7] T. Behnke et al. (editors), The International Linear Collider Technical Design Report Volume 1: Executive Summary, arXiv:1306.6327

[8] G. Contin, the STAR collaboration, The STAR PXL detector, 2016 JINST 11 C12068

[9] M. Deveaux et al., Design considerations for the Micro Vertex Detector of the Compressed Baryonic Matter experiment, PoS Vertex2008 (2008) 028

[10] M. Deveaux, J. Heuser, The silicon detector systems of the Compressed Baryonic Matter experiment, PoS Vertex2013 (2013) 009

[11] M. Koziel, The prototype of the Micro Vertex Detector of the CBM experiment, NIM-A Vol. 732 (2013) P515-518

[12] The NA61/SHINE Collaboration, Addendum to the the NA61/SHINE Proposal SPSC$P$-330, Beam momentum scan with Pb+Pb collisions by NA61/SHINE at the CERN SPS, CERN-SPSC-2015-038 / SPSC-P-330-ADD-8 15/10/2015

[13] The NA61/SHINE Collaboration, Status report to the proposal SPSC-P-330 Report from the NA61/SHINE experiment at the CERN SPS, CERN-SPSC-2016-038 / SPSC-SR-197 $08 / 10 / 2016$

[14] M. Deveaux et al., The Small Acceptance Vertex Dtector of NA61/SHINE, EPJ Web of Conferences 171, 21001 (2018)

[15] J. R. Schwank et al., Radiation Effects in MOS Oxides, IEEE TNS Vol. 55, No 5, P. 1833 (2008) 
[16] M. Suljic, ALPIDE: the Monolithic Active Pixel Sensor for the ALICE ITS upgrade, Journal of Instrumentation 11.11 (2016): C11025.

[17] U. Berdalovic et al., Monolithic pixel development in TowerHazz $180 \mathrm{~nm}$ CMOS for the outer pixel layers in the ATLAS experiment 2018 JINST 13 C01023

[18] H. Augustin et al., The MuPix system-on-chip for the Mu3e experiment, NIM-A 845 (2017) P. 194-197

[19] M. Benoit et al., Testbeam results of irradiated ams H18 HV-CMOS pixel sensor prototypes, JINST 13 P02011

[20] T. Wang et al., Depleted fully monolithic CMOS pixel detectors using a column based readout architecture for the ATLAS Inner Tracker upgrade, JINST 13 C03039

[21] N. S. Saks et al., Generation of interface states by ionizing radiation in very thin MOS oxides, IEEE TNS Vol. NS-33, No. 6, P. 1185, Dec. 1986

[22] G. Lutz, Semiconductor Radiation Detectors, Springer (1999), ISBN 3-540-64859-3

[23] H. Hillemanns et al., Radiation hardness and detector performance of new 180nm CMOS MAPS prototype test structures developed for the upgrade of the ALICE Inner Tracking System, Nuclear Science Symposium and Medical Imaging Conference (NSS/MIC), 2013 IEEE. IEEE, 2013

[24] P.Jarron et al. , Deep submicron CMOS technologies for the LHC experiments, Nuclear Physics B (Proc. Suppl.) 78 (1999) 625-634

[25] W. Snoeys et al., Layout techniques to enhance the radiation tolerance of standard CMOS technologies demonstrated on a pixel detector readout chip, NIM-A 439 (2000) 349-360

[26] G. P. Summers et al., Damage correlations in semiconductors exposed to gamma, electron and proton radiations, IEEE TNS Vol. 40, No. 4, P. 1372 (1993)

[27] A. Vasilescu et al., The NIEL scaling hypothesis applied to neutron spectra of irradiation facilities in the ATLAS and CMS SCT, ROSE/TN/97-2, CERN (1997)

[28] A. Vasilescu and G. Lindstroem, Displacement damage in silicon, on-line compilation, http://rd50.web.cern.ch/RD50/NIEL/default.html

[29] G. Lindstroem, Radiation damage in silicon detectors, NIM-A 512.1 (2003): 30-43.

[30] S. W. Glunz et al., Minority carrier lifetime degradation in boron-doped Czochralski silicon, Journal of applied physics, Vol. 90 Nb. 5, P. 2397 (2001)

[31] H.W. Kraner, Radiation damage in silicon detectors, NIM-A 225 (1984) 615-618

[32] V. Cindro et al., Radiation damage in p-type silicon irradiated with neutrons and protons, NIM-A 599 (2009) 60-65 
[33] R. Wunstorf et al., Investigations of donor and acceptor removal and long term annealing in silicon with different boron/phosphorus ratios, NIM A 377 (1996) 288-233

[34] I. Mandic et al., Neutron irradiation test of depleted CMOS pixel detector prototypes, Journal of Instrumentation 12.02 (2017): P02021.

[35] A. Affolder et al., Charge collection studies in irradiated HV-CMOS particle detectors, Journal of Instrumentation 11 (2016) P04007.

[36] A. Potenza et al., Radiation tolerance of a moderate resistivity substrate in a modern CMOS process, NIM-A 718(2013)347-349.

[37] B. Hiti et al., Charge collection properties in an irradiated pixel sensor built in a thick-film HV-SOI process, 2017 JINST 12 P10020.

[38] B. Hiti et al., Charge collection in irradiated HV-CMOS detectors, NIM A (2018), https://doi.org/10.1016/j.nima.2018.07.022.

[39] S. M. Sze and Kowk K. Ng, Physics of semiconductor devices, third edition, John Wiley \& Sons, INC. 2007, ISBN 978-0-471-14323-9.

[40] G. Deptuch, New Generation of Monolithic Active Pixel Sensors for Charged Particle Detection, doctoral thesis, ULP Strasbourg (2002).

[41] G. Deptuch, et al., Monolithic active pixel sensors with in-pixel double sampling operation and column-level discrimination., IEEE TNS 51.5 (2004): 2313-2321.

[42] Y. Degerli et al., A fast monolithic active pixel sensor with pixel-level reset noise suppression and binary outputs for charged particle detection, IEEE - TNS, Vol. 53, Iss. 6, P. $3186(2005)$.

[43] M. Deveaux et al., Radiation tolerance of a column parallel CMOS sensor with high resistivity epitaxial layer, JINST 6.02 (2011): C02004.

[44] A. Besson et al., A vertex detector for the International Linear Collider based on CMOS sensors, NIM-A, Vol. 568, Iss. 1 (2006) P. 185-190.

[45] P. Roloff, The EUDET high resolution pixel telescope, NIM-A Vol. 604, Iss. 1-2 (2009) P265-268.

[46] C. Hu-Guo et al., First reticule size MAPS with digital output and integrated zero suppression for the EUDET-JHRA1 beam telescope, NIM-A, Vol. 623, Iss.1, P. 480-482 (2010).

[47] J. Baudot et al, First Test Results Of MIMOSA-26, A Fast CMOS Sensor With Integrated Zero Suppression And Digitized Output, IEEE Nuclear Science Symposium Conference Record (NSS/MIC) (2009).

[48] I. Valin, et al., A reticle size CMOS pixel sensor dedicated to the STAR HFT 2012 JINST $7 \mathrm{C} 01102$. 
[49] G. Contin et al., The STAR MAPS-based PiXel detector, NIM-A Vol. 907 (2018) P. 60-80.

[50] M. Deveaux, Development of fast and radiation hard Monolithic Active Pixel Sensors (MAPS) optimized for open charm meson detection with the CBM - vertex detector, PhDThesis, University Louis Pasteur Strasbourg, Goethe University Frankfurt (2008).

[51] K. Dette, Total Ionising Dose effects in the FE-I4 front-end chip of the ATLAS Pixel IBL detector, 2016 JINST 11 C11028.

[52] D. Doering, Untersuchungen zur Verbesserung der Strahlenhärte von CMOS-Sensoren zum Einsatz in Vertexdetektoren von Schwerionenexperimenten, $\mathrm{PhD}$ thesis, Goethe University Frankfurt, 2014, in German.

[53] L. Jungermann, Elektronik für das Weltraum-Experiment AMS02 und Bildgebung in der Strahlenmedizin, PhD-Thesis, TU Karlsruhe (2005), P138.

[54] M. Deveaux et al., Charge Collection properties of X-ray irradiated monolithic active pixel sensors, NIM-A 552 (2005) 118-123.

[55] El-Sayed Eid et al., Design and Characterization of Ionizing Radiation-Tolerant CMOS APS Image Sensors up to $30 \mathrm{Mrd}$ (Si) Total Dose, IEEE TNS Vol. 48, No. 6, P1796 (2001).

[56] Deveaux, M., et al. Radiation tolerance of CMOS monolithic active pixel sensors with self-biased pixels. NIM-A 624.2 (2010): 428-431.

[57] S. Amar-Youcef, Design and performance studies of the Micro-Vertex-Detector for the CBM experiment at FAIR, $\mathrm{PhD}$ thesis, Goethe University Frankfurt (2011).

[58] M. Koziel, Development of radiation hardened pixel sensors for charged particle detection, PhD Thesis, Université de Strasbourg (2011).

[59] A. Besson, From vertex detectors to inner trackers with CMOS pixel sensors, NIM-A 845 (2017) 33-37.

[60] A. Shabetai, Mesure de la production de charme ouvert dans les collisions d'ions lourds avec l'expérience STAR au RHIC. Etude prospective de l'apport du "Heavy Flavor Tracker" utilisant des capteurs CMOS, PhD Thesis, Université Louis Pasteuer Strasbourg, 2008.

[61] M. Szelezniak, Development of pixel detectors with in itegrated microcircuits for the Vertex Detector in the STAR experiment at the RHIC collider, $\mathrm{PhD}$ Thesis, Université Louis Pasteuer Strasbourg, 2008.

[62] A. Dorokhov et al., High resistivity CMOS pixel sensors an their application to the STAR PXL detector, NIM-A Vol 650 (2011) P174-177.

[63] A. Dorokhov et al., Improved radiation tolerance of MAPS using a depleted epitaxial layer, NIM-A Vol. 624, Iss. 2, P. 432 (2010) 
[64] M. Deveaux et al., Neutron radiation hardness of monolithic active pixel sensors for charged particle tracking, NIM-A Vol. 512, Iss. 1-2, P71-76 (2003)

[65] G. Deptuch et al., Simulation and measurements of charge collection in monolithic active pixel sensors, NIM-A 465 (2001) 92-100

[66] J.J. Velthuis et al., Characterization of Active Pixel Sensors in $0.25 \mu \mathrm{m}$ CMOS Technology, IEEE TNS Vol. 52, NO. 5, P1887 (2005)

[67] A. Budenbender, Systematische Untersuchungen der Strahlentoleranz optimierter Monolithic Active Pixel sensoren gegen nicht-ionisierende Strahlung, Bachelor-Thesis, Goethe University Frankfurt 2008, in German.

[68] T. Bus, B. Linnik, A. Yazgili, Goethe University Frankfurt, personal communication.

[69] Yu. Gornushin et al., Tracking performance and radiation tolerance of monolithic active pixel sensors, NIM-A 513 (2003) 291?295

[70] M. Deveaux et al., Charge collection properties of Monolithic Active Pixel Sensors (MAPS) irradiated with non-ionising radiation, NIM-A Vol 583, Iss. 1, P. 137 (2007)

[71] D. Doering et al., Pitch dependence of the tolerance of CMOS monolithic active pixel sensors to non-ionizing radiation, NIM-A Vol. 730, P. 111-114 (2013)

[72] A. Besson et al., Achievements of CMOS Pixel Sensors for the CBM Micro-Vertex Detector, CBM Progress Report 2007, P. 30, ISBN: 978-3-9811298-5-4

[73] B. Linnik, PhD, Goethe University Frankfurt am Main, under preparation.

[74] Process and Device Simulation Tools, Synopsys, Inc., Mountain View, California

[75] S. Strohauer, Strahlenhärte eines hochohmigen CMOS Monolithic Active Pixel Sensors mit bis zu $80 \mu \mathrm{m}$ Pixelpitch gegen nicht-ionisierende Strahlung, Bachelor Thesis, Goethe University Frankfurt 2014, in German

[76] J. Baudot, Optimization of CMOS pixel sensors for high performance vertexing and traking, NIM-A Vol. 732 (2013) P. 480-483

[77] J. Heymes, IPHC Strasbourg, personal communication.

[78] B. Linnik et al., Radiation hardness of MAPS for CBM - A first look on AC-coupling. Presentation given on the 30th CBM Collaboration Meeting, Sept. 24 - Sept. 28, 2017, Wuhan, China.

[79] J. Heymes et al., Study of the depletion depth in a frontside biased CMOS pixel sensors, 2019 JINST 14 P01018

[80] D. Doering, Ausheilstudien an bestrahlten Monolithic Active Pixel Sensoren, Master Thesis, Goethe University Frankfurt (2010), in German. 
[81] D. Doering, Annealing studies on X-ray and neutron irradiated CMOS Monolithic Active Pixel Sensors, NIM-A 658 (2011) 133-136

[82] D. Doering, Goethe University Frankfurt, now Zalaris Deutschland AG, personal communication

[83] D. Doering, Random Telegraph Signal in Monolithic Active Pixel Sensors, Bachelor Thesis, Goethe University Frankfurt (2008), in German

[84] J. Bogaerts, B. Dierickx, R. Mertens, Random Telegraph Signals in a Radiation-Hardened CMOS Active Pixel Sensor, IEEE Trans. Nucl. Sci. 49, 249 (2002).

[85] C. Virmontois et al., Total Ionizing Dose Versus Displacement Damage Dose Induced Dark Current Random Telegraph Signals in CMOS Image Sensors, IEEE Transactions on Nuclear Science 58.6 (2011): 3085-3094.

[86] V. Goiffon et al., Multi level RTS in proton irradiated CMOS image sensors manufactured in a deep submicron technology, IEEE Trans. Nucl. Sci., vol. 56, no. 4, pp. 2132-2141, Aug. 2009 .

[87] G. R. Hopkinson, A. Mohammadzadeh, R- Harboe-Sorensen, Radiation Effects on a Radiation-Tolerant CMOS Active Pixel Sensor, IEEE Trans. Nucl. Sci. 51, 249 (2004).

[88] G. R. Hopkinson, Radiation Effects in a CMOS Active Pixel Sensor, IEEE Trans. Nucl. Sci. 47, 2480 (2000).

[89] M. Deveaux et al., Random telegraph signal in monolithic active pixel sensors, Nuclear Science Symposium Conference Record, 2008. NSS'08. IEEE. IEEE, 2008.

[90] Ming-Horn Tsai and Tso-Ping Ma, The Impact of Device Scaling on the Current Fluctuations in MOSFET's, IEEE Transactions on electron devices, Vol. 41, No. 11, P. 2061, (2004)

[91] P. Martin-Gonthier et al., In-Pixel Source Follower Transistor RTS Noise Behavior Under Ionizing Radiation in CMOS Image Sensors, IEEE Transactions on Electron Devices, vol. 59 (No 6). P. 1686-1692 (2012)

[92] A. Asenov et al., RTS Amplitudes in Decananometer MOSFETs: 3-D Simulation Study, IEEE Transactions on electron devices, VOL. 50, NO. 3, P 839, (2003)

[93] Wang, Xinyang et al., Random telegraph signal in CMOS image sensor pixels., Electron Devices Meeting, 2006. IEDM'06. International. IEEE, 2006.

[94] D. Doering et al., Noise performance and ionizing radiation tolerance of CMOS Monolithic Active Pixel Sensors using the 0.18 m CMOS process, 2014 JINST 9 C05051

[95] P. Martin-Gonthier et al., RTS Noise Impact in CMOS Image Sensors Readout Circuit., 16th IEEE International Conference on Electronics, Circuits, and Systems, 2009, ICECS 2009, Hammamet, Tunisia, 13-16 December 2009. Piscataway NJ : IEEE, 2010, pp. 928931. ISBN 978-1-4244-5090-9 
[96] The ALICE collaboration, Technical Design Report for the Upgrade of the ALICE Inner Tracking System, 2014 J. Phys. G: Nucl. Part. Phys. 41087002

[97] H. Muto et al., Random Telegraph signal in Small MOSFET's After X-ray Irradiation, IEEE TNS Vol. 38, No. 6, P. 1116 (1991)

[98] I. Mandic et al. Bulk damage in DMILL npn bipolar transistors caused by thermal neutrons versus protons and fast neutrons. IEEE Transactions on Nuclear Science 51.4 (2004): $1752-1758$.

[99] I. Mandic et al. Radiation damage in bipolar transistors caused by thermal neutrons. Nuclear Science Symposium Conference Record, 2003 IEEE. Vol. 1. IEEE, 2003.

[100] B. Linnik et al. Radiation damage caused by cold neutrons in boron doped CMOS active pixel sensors, Journal of Instrumentation 12.05 (2017): C05011.

[101] J.F. Ziegler and J: P. Biersack. The stopping and range of ions in matter. Treatise on Heavy-Ion Science, Springer US, 1985. 93-129.

[102] H. Breitkreutz et al. Spectral fluence rates of the fast reactor neutron beam MedApp at FRM II. NIM-A 593.3 (2008): 466-471.

[103] Heinz Maier-Leibnitz Zentrum. (2015). PGAA: Prompt gamma and in-beam neutron activation analysis facility. Journal of large-scale research facilities, 1, A20. http://dx.doi.org/10.17815/jlsrf-1-46 .

[104] G. Contin on behalf of the STAR Collaboration, The STAR PXL detector, 2016 JINST $11 \mathrm{C} 12068$.

[105] G. Contin et al., The STAR MAPS-base PiXeL detector, NIM-A Vol. 907, P. 60-80 (2018)

[106] S.R. Messenger et al., NIEL for Heavy Ions: An Analytical Appraoch, IEEE TNS Vol. 50 (2003) 1919.

[107] M. Kurokawa et al., Radiation damage factor for ion implanted silicon detectors irradiated with heavy ions, IEEE TNS 42 (1995) 163.

[108] V. Eremin et al., A comparative study of silicon detector degradation under irradiation by heavy ions and relativistic protons, 2018 JINST 13 P01019.

[109] M. A. Xapsos, et al., NIEL calculations for high-energy heavy ions, IEEE TNS Vol. 51 (2004), P. 3250-3254.

[110] H. Augustin et al., Irradiation study of a fully monolithic HV-CMOS pixel sensor design in AMS $180 \mathrm{~nm}$, NIM-A 905(2018) 53-60

[111] H. Pernegger et al., First tests of a novel radiation hard CMOS sensor process for Depleted Monolithic Active Pixel Sensors, 2017 JINST 12 P06008 